\title{
Author Index to Abstracts
}

A

Abadeer, RA................................ 929

Abal, L ……………………...... 492

Abbott, D.................................... 469

Abdalla, EK …………………..... 1452

Abdolell, M ………………….....275

Abdul-Karim, FW …………....... 1649

Abe, H................................... 1404

Abendroth, CS ............................... 422

Abi Daoud, MS ………………..... 437

Abi-Raad, R ................................ 148

Abonnet, V ............................... 1600

Abouljoud, M............................ 1449

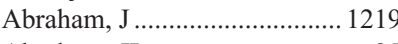

Abraham, JL..............................25

Abraham, KP............................... 426

Abraham, SC.................1386, 1480

Abrahams, R ………………….... 626

Abromowitch, M....................... 1268

Abruzzo, LV ………......1217, 1289

Absi, R ....................................... 39

Abu Shahin, F ...................930, 931

Abu-Asab, M............................. 1779

Abudayyeh, S............................573

Abudul-Karim, F............... 930, 931

Abu-Farsakh, HA ........................ 352

Aburatani, H.............................. 1030

Achacoso, N ....................... 143, 144

Ackerman, AM......................... 1299

Acon Laws, M................................535

Acosta Haab, G .......................... 168

Acquafondata, M............................... 782

Acquafondata, MB .................... 750

Acs, G ......... 40, 176, 243, 318, 456

Acurio, A...................... 1110, 1576

Adamiak, A ………………….... 932

Adams, A.....................................229

Adams, AL ……………....104, 278

Adams, CD....................1364, 1368

Adams, RB .......................... 1440

Adamson, CSC........366, 1119, 1144

Adegboyega, PA........................ 1053

Adelstein, D ............................. 1129

Ademuyiwa, FO............................ 312

Adeniran, A …………………........ 696

Adeyi, O...................................... 1411

Adeyi, OA ………….....1387, 1459

Adkins, CB.................................. 196

Adrover, E.........................261, 270

Adsay, N................229, 1443, 1444

Adsay, NV .............. 672, 807, 1392

$1398,1399,1465$

Advani, R ................................ 1249

Agaram, NP..............34, 629, 1577

Aggarwal, B …………………..... 596

Agni, RM …………………......... 605

Agoston, AT ………………….......598

Agoston, EA................................1111

Agrawal, D ……………………... 519

Agrawal, M ………………….... 82

Aguayo, FR …………………. 56

Aguilar, CE ……………...542, 1423

Aguilera, N............................. 1325

Aguilera-Barrantes, I..........697, 933

Aharonov, R ..................1630, 170

Aherne, S...................513, 521, 970

Aherne, ST ................................. 511

Ahiskali, R ................................966

Ahmed, IT .................................... 241

Ahmed, Q.................................... 189
Ahn, JH ..................................... 543

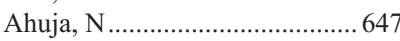

Aisa, G ........................................ 1626

Aisner, S..................................... 1311

Ajani, JA ……………………...... 625

Akada, M.................................... 1463

Akaska, T .................................. 1279

Akgun, H......................................

Akhtar-Danesh, N .............. 160, 161

Akiyama, T.............................. 1286

Akporiaye, E …………………......... 495

Akram, M.................................. 326

Al Habeeb, A............................... 1714

Alaggio, R ................. 89, 791, 1562

Al-Ahmadie, H.................. 805, 880,

$896,897,1180$

AlAllawi, N.............................. 1322

Alam, SM ........................................ 360

Alani, RM …………………...... 484

Alaoui-Jamali, MA...................... 742

Alarcon, G................................ 1442

Alatassi, H.............. 373, 1474, 1625

Albadine, R ............... 698, 699, 700

Albadine, r.................................. 785

Albadine, R ....... 786, 803, 813, 844 , $894,895,908,1484$

Albanese, J ................................. 1156

Albarello, L .................................512

Albarracin, CT ...................626, 1492

Albequerque, $\mathrm{K}$.............................233

Alberti, N ..................................... 105

Albores-Saavedra, J ....................545

Alcaraz, E.................. 273, 286, 546

Aldape, K ……………………...... 876

Aldape, KD .................................. 862

Al-Daraji, W............................... 1336

Alejo, M..........738, 739, 974, 1026

Alemany, L..............738, 739, 1026

Alenda, C .......... 110, 261, 286, 546

Alexander, J ..................1397, 1414

Alexander, S................... 1314, 1584

Alexis, J........................271, 1725

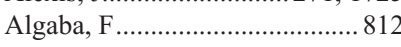

Algara, P....................... 1157, 1283

Al-Ghawi, H............ 544, 863, 1573

Ali, L ........................................ 701

Ali, S ......................................... 387

Ali-Fehmi, R ............. 936, 939, 982

Alikhan, MB ……………….... 1578

Al-Jalad, MA ....………………............. 352

Alkan, S....... 522, 1154, 1251, 1718

Alkushi, A ................................. 934

Allan, RW.........................720, 725,

$726,946,1579$

Allen, C ................................... 1715

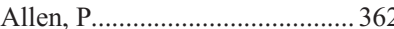

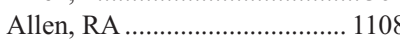

Allen, TC...................... 1580, 1581

Allende, DS .............................. 1388

Allison, KH ............... 106, 183, 935

Allison, MJ.............................. 1327

Allitto, BA..................................... 597

Allory, Y.................................... 853

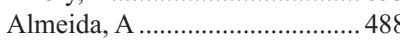

Almiski, M ........................... 1155

Alobeid, B .....................1244, 1293,

Alonso, V ................................... 475

Alos, L........................... 1112, 1332

Alpers, CE................................ 1379

Al-Quran, SZ......... 720, 1150, 1165
Alrawi, S ........................ 675, 1609

Alsaad, K.................................. 1375

Alsabeh, R............................. 1232

Altendorf-Hofmann, A ...................57

Alter, BP.................................. 1254

Althof, PA..................................... 103

Altinel, D........................672, 1398

Altiok, S .................................. 1673

Alvarado-Cabrero, I ............ 107, 108

Alvarez, A …………………....702

Alvarez, K............... 875, 919, 1690

Alves, VAF .......................695, 1552

Alves-ferreira, J.......................... 1288

Amar, M ................................. 585

Amat, L ……………………..... 848

Ambroisine, L …………………. 86

Amer, HZ …………………...... 1521

Amin, M........... 153, 430, 441, 703,

$704,712,819,820$

$825,840,1270,1558$

Amin, MB ............... 564, 703, 705,

$711,712,721$

$776,819,840,85$

Amin, W.................................. 1716

Amorim, J ..................................1506

Anagnostou, VK...…………..... 1717

Anatelli, F ………………….... 1113

Andea, A.................... 438, 666, 706

Anders, RA....................................56

Anderson, M ............................. 1618

Anderson, ME ................................. 73

Anderson, MP .......................... 1491

Anderson, S................................316

Anderson, SS..................... 118, 251

Andersson, SO ……….......760, 885

Andersson, T ................................ 149

Andino, L …………………..... 707

Ando, M .....................................203

Andraws, N ………………….... 924

Andren, O..........................760, 885

Andres, A.................................... 1433

Andrulis, IL ..................117, 248

Andrus, C ....................................207

Andry, C................................. 1114

Andry, CD ………………………... 2

Ang, L ........................................ 474

Angelidou, S .............................. 1011

Angelov, L........................................... 1512

Angulo, JC …………………..... 810

Anlauf, M..................................... 533

Ansell, S .............................. 1317

Ansell, SM ................................ 1279

Antoine, M …...................109, 125

Anton, I ...................................... 811

Antonello, Z ………………......... 528

Antonescu, CR ……………...34, 87

Antunez, P................................... 487

Aoun, P................ 1201, 1258, 1264

Apple, SK .................................... 236

Arabi, H...........................936, 982

Arabi, MH .................................. 939

Arai, C..................................... 1482

Arai, M................................... 1624

Araki, M...................................... 901

Aranda, FI ............... 110, 228, 261 ,

$270,273,286$

Araujo, ARC ............................ 1040

Arber, DA.. 1177, 1256, 1315, 1316

Arcarese, B.................. 1652, 1658

Arce, J ................................... 1156

Archibald, TA................................... 353
Arcila, M................................... 1390

Arenillas, L...................................... 1167

Argani, P .......... 140, 306, 313, 700 , $821,1545,1554,1563$

Arguelles, C ……………….......678

Argueta, V .................................. 1343

Arlinghaus, RB …………….... 1533

Armitage, J ............................... 1263

Armstrong, S ............................ 1280

Arnould, L ..................................281

Arranz, I ...................................... 120

Arribas, A _........................... 1226

Arribas, JA ......................................... 1157

Arrinda, J..................................1516

Arrive, L...................................... 631

Arseneau, J................................ 1007

Arthur, D …………………...... 1254

Arya, S ................................... 1114

Asa, SL.......... 534, 954, 1498, 1732

Asano, S .................................... 1328

Asanuma, H.................................. 74

Asgari, M ................................ 1361

Ashby-Richardson, H....... 111, 654

1522

Ashfaq, R .........................193, 198

Ashikaga, T .............................2230

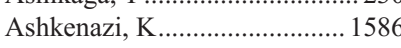

Ashworth, A _......175, 240, 244

$254,255,277,1506$

Askari, S...................................... 547

Aspden, L ...................... 1142, 1594

Aspden, LM ………………….... 526

Astbury, K ................................937

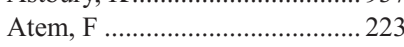

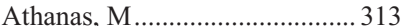

Athanassiadis, A........................ 1550

Atieh, M ……………………..... 702

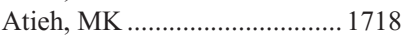

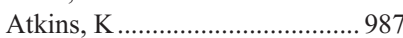

Atkins, KA ……...............170, 368

Atkinson, B …………………...... 1608

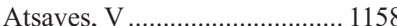

Attygalle, A ...................................... 1281

Aubry, MC ………….... 1634, 1640

Auerbach, A........................ 46, 1325,

Auerbach, J ……………….......... 112

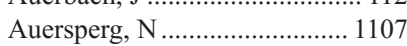

Aulmann, S …………..... 297, 1563

Austin, RM.......................... 436, 989

Autonell, J .......................... 660

Avril, MF........................................ 488

Axelson, G …………………...... 938

Axiotis, CA ……………………... 303

Ayala, AG ......................... 769, 874

$875,1450,1690$

Ayala, E...................................... 848

Ayala, G .......................... 848, 881

Ayaquica, C.................................. 127

Aydin, H........................... 708, 709

Aydin, O .................................. 1402

Ayers, L L...………………….... 1170

Azar, DW .......................................

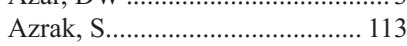

Azuma, K ………………….... 1778

Azumi, N.................................... 1520

B

Baba, Y..............................638, 643

Babbin, B ……………………...... 314 
Bacchi, CE ....................... 153, 705 Bacchini, P .................................. 97 Bacigalupo, B............................. 166 Badve, S .................. 114, 115, 312 Bae, HI .............................. 377, 606 Bae, H-I...................................... 595 Bae, JH.................................. 1001 Bae, YK..................... 216, 586, 595 Baehner, FL...............114, 115, 116 Baer, H ........................................ 320 Baergen, RN............................. 1572

Bagg, A...................................... 1231 Bahler, DW ......... 1159, 1187, 1346 Bahlis, N ..................... 1253, 1691

Bahrami, A .................................... 35

Bahrami, S.................................. 486

Bai, S................................36, 710

Bai, Y ..................................... 1667

Bailey, D ………….......... 760, 902

Bakeman, W ................................ 990

Baker, K ……………………....239

Baker, ML ………………......... 1719

Baker, P .................. 19, 1007, 1350

Baker, SE ......................... 354, 355

Bakhiet, SM …………………. 1720

Bakst, JB …………................. 1742

Balague, O.....................1160, 1265

Balasubramaniam, R ..................548

Balaton, A.......................281, 1357

Baldi, E ……………………...... 842

Baldini, EH …………........ 76, 247

Ball, MW.......................................... 908

Balla, A.......................................... 817

Baloch, ZB ………………….... 1126

Baloch, ZW ........... 367, 1138, 1147

Balog, CI.................................. 100

Balos, L ........................................ 1558

Balsalobre, FA........................... 1627

Balzer, B.......................... 711, 712

Balzer, BL …………………........564

Bandarchi, B ............................. 409

Bandyopadhyay, S.............936, 939,

982, 1392

$1443,1444,1465$

Bane, AL …............................... 117

Bane, BA...................................... 1108

Banerjee, H ……………............. 190

Banerjee, P …………….. 713, 1484

Banerjee, S ................................. 1674

Bangma, C.................................... 727

Banjo, K .................................... 1026

Bannykh, S.............................. 1518

Banos, A .................................... 811

Bansal, I .......................... 1168

Bansal, M ……………….... 356, 357

Banville, NM............................ 1115

Bao, F........ 1389, 1390, 1391, 1461

Bao, J...................................... 1695

Baqai, J..................................... 940

Barakat, R ................................... 978

Baran, CE .................................... 439

Barbashina, V .............................. 327

Barbera, V ……………………....546

Barbi, S ................................... 1711

Barbuto, D...................... 1094, 1096

Barbuto, DA ................................ 1099

Barkan, G......................... 433, 702

Barkan, GA ……………...374, 379 ,

522, 770, 1718

Barkoh, B ….......................... 1773 Barletta, JA 1582, 1583, 1635, 1683 Barlogie, B ………………….... 1168

Barner, R ................................. 1105

Barnes, EL...................... 1127, 1128
Barnes, PJ................................... 275 Barqawi, A..................................... 694 Barr, T ..................................... 1350 Barreto, JE.................................... 848

Barrett, C..................................... 714 Barrett, M........................ 358, 1278 Barrette, S ................................... 39 Barr-Fritcher, EG ………………..... 118 Barrios, R .............................. 1380

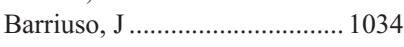
Barroso-Bravo, S...……………. 107 Barry, M ………………………....20 Barshack, I .....................763, 1630 Barshak, I ................................ 1701 Barsky, SH .... 119, 293, 1529, 1687 Bartels, CL ......... 1719, 1737, 1755 Bartlett, AM ............................... 1523 Bartlett, D.......................... 79, 611

Bartley, AN ................................ 549 Bartoletti, R.................................. 841

Barton, JH ......................... 791, 890

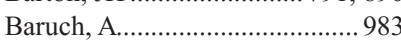

Barzon, L.................................... 722

Bas, E ………………………..... 966

Basbug, M................................... 1

Basrsky, SH...................................... 1551

Basrur, V …………….... 1179, 1681

Basso, C ……………………...... 337

Bast, RC …………………......... 1107

Bastacky, S..............................429

Basturk, O ........... 672, 1392, 1399 ,

$1443,1444,1465$

Basu, A ................................... 1329

Batal, I.................................... 1329

Batich, K ……………………... 545

Batra, SK …………….... 980, 1059

Batten, JM ...................................... 78

Bauer, TW …………………….... 1440

Bauersfeld, J............................. 1668

Baumann, M................................60

Baumert, H.............................. 1357

Bautista, D.................................. 120

Bayindir, P............................. 1775

Baytur, Y ……………………….... 23

Baz, MA ....................................1579

Bazarbachi, A ............................ 1285

Beam, CA ……………………..... 817

Bean, SM....................................... 501

Bearzi, I.......................................... 661

Beaty, MW ………………….... 1161

Beaubier, NT .................. 1485, 1584

Becette, V.................................. 125

Bechert, CJ......................... 941, 942

Becich, MJ ............................... 1716

Beck, AH............................. 289, 1669

Beck, S ................................... 1692

Beckman, EN ………………...... 1330

Bedrossian, K............................... 385

Beebe, K.................................. 1311

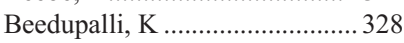

Beer, NL .................................... 362 Begley, H......................... 1062, 1704 Begnami, MD............................. 550

Behar, J..................................... 1537

Beher, J................................... 1723

Beheshti, J ......................551, 1585

Behm, J .................................. 1077

Behmoaram, E...……………...... 742

Behrens, C................................... 1643

Beitler, JJ..................................... 378

Bejarano, PA.................. 1116, 1393

Belanger, GV.............................. 1739

Belikoff, BG.............................. 1530

Bell, D..
Bell, DA

Bell, KB ...

Bell, WC

Bellamy, W.

Bellas, C

Bellis, M...

Bellizzi, AM........

Bellizzi, SM

1395,1481

.

Bellolio, E ................................. 1392

Belsley, NA …………………...... 121

Belt, P......................................... 1476

Bemis, L .................................... 1617

Benedettini, E................. 1651, 1670

Benevenia, J ............................... 1311

Ben-Ezra, JM ………………....1342

Benjamin, H ............................. 1586

Bennett, AE ………….......569, 656

Benstein, BD ............................... 940

Bentley, G …………………..... 1408

Bentley, HA................................. 1740

Bentwich, Z.........1586, 1630, 1701

Bentz, J..................................... 359

Berean, KW......................................... 1121

Berenson, M ............................... 323

Berg, JC......................................... 440

Bergeron, C ................................. 962

Berghuis, D …………………….... 37

Berho, M ..................................... 552

Beriwal, S................................... 123

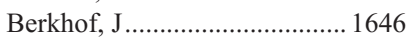

Berkowitz, RS ............................ 972

Berman, DM .............................. 803

Bermingham, NJ ..................... 1115

Bermudo, R …………………..... 167

Bernacki, EG............................. 1558

Bernadt, C ............................... 1654

Bernal, ML .................................... 120

Berndt, A ....................................... 57

Berney, D ……………………... 812

Berney, DM …………….....715, 861

Beroukhim, R........................... 1677

Berry, G.................................... 1736

Bertacca, G.................................. 747

Berteau, P................................. 125

Berthier, CC ………………....... 1372

Bertolaso, A........................................ 1711

Bertoni, F ................................97

Besse, P ...................................... 227

Bevilacqua, G............................... 747

Bewtra, C

Bhagat, $\mathrm{G}$

..... 678,1244

$1293,1314,1321$

Bhagavathi, S ........441, 1270, 1676

Bhargava, R...... 122, 123, 138, 178 , $179,180,259,264$

Bhasin, M..................................... 86

Bhat, R ………………….... 5, 124

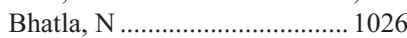

Bhatnagar, J............................. 1333

Bhattacharjee, MB …………..... 1486

Bhattacharyya, A ..........................549

Bhattacharyya, AK …………...... 507

Bhavnani, S .............................. 1372

Bhorade, S................... 1591, 1605

Bhorade, SM …………………... 1644

Bianco, FJ ......................... 838, 839

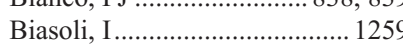

Bibeau, F ................................. 125

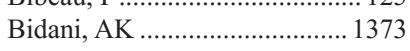

Bielecki, LC .............................1627

Bien-Willner, GA ...................... 1486

Bifulco, C ............................. 1235

Bijian, K ……………………..... 742

Bilbao, FJ ..................................... 1118

Bilbao, J ................................... 1118 Billings, SD ........................ 94, 493 Billis, A ..... 700, 705, 716, 717, 860 Billiter, D ................................. 1350 Bilowus, M........................................ 694 Binder, SW ............................... 482

Bing, Z ……………………..... 718

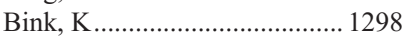

Birbe, R ..................................... 681

Birch, C ............................

Bird, B B...…………………...... 385

Bird, BL ..................................... 111

Bird, V............................... 780, 780

Birdsong, G ........................132, 404

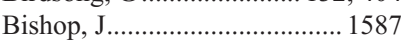

Bishu, S..........................1564

Bismar, T.................................... 903

Bismar, TA ............. 742, 743, 1674

Bisson, MAM.....................717, 860

Bjornstedt, M ........................... 1672

Blackford, A ................................1396

Black-Schaffer, WS......................... 426

Blanco, JL .............................. 1436

Blanes, A ................... 459, 516, 537

Bleiweiss, IJ ................................ 206

Blenc, AM ....................... 441, 1270

Bloch, NB ……………..... 753, 767

Blochin, E ....... 442, 579, 719, 1407

Bloom, G.................................. 149

Bloom, K................................. 1758

Bloom, KJ ......................... 126, 553

Bloom, L ................................... 940

Bloom, TA........................ 126, 553

Bloomfield, CD ......................... 1208

Bloomston, M ................620, 1394

1395,1481

Blount, PL ................................... 567

Blue Arm, N ......................................... 6

Blum, MG ............................... 1628

Blumgart, LH ............................ 1389

Bociek, G ............................... 1201

Bocon-Gibod, L ……………....... 812

Bodo, J ……………………...... 1284

Boerner, S..................................... 423

Boers, JE ................................... 554

Boesel, C ................................. 1446

Bogner, PN .......................................... 469

Boguniewicz, AB ......283, 300, 301

Bohn, OL.................................. 127

Boissiere-Michot, F..................... 125

Boisvert, SL ………………....... 1009

Boitier, F ..................................... 488

Bojakowski, K.............................. 344

Bojarski, E..................................... 578

Boland, JM..................................... 38

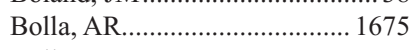

Bollen, AW ................................. 992

Bolshakov, S ................................. 64

Bolster, MA ....................................22 22

Bolton, BD ............................... 1564

Bombonati, A ....................................... 943

Bomeisl, PE.............................. 360

Bonaccorsi, L ............................ 842

Bonaventura, M............................209 
Bose, S ..... 151, 369, 394, 395, 425, $427,465,944,977,1733$

Bosincu, L ................................ 1010 Bosler, DS ................... 1162, 1163

Bostwick, DG...................694, 904

1539,1548

Botrell, A .................................... 137

Bott, C ............................................ 1382

Botta, C ................................... 240

Boulos, FI.................................... 129

Boulware, D …………………..... 593

Bouron-Dal Soglio, D ………….... 39

Bouse, CF...................................350

Boutrus, R ……………………... 148

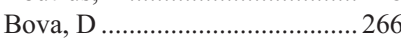

Bove, KE................................1507

Bovee, JV ..............................97, 98

Bovee, JVMG …………………... 93

Bovio, IM ……………..... 720, 1579

Boyd, SD .................................167

Boyd, TK ……………...1574, 1575

Boyd, W ................................. 1088

Boyle, SH ..................................225

Boyum, RD ………………............. 1721

Bozkurt, S …………………. 115

Bozkurt, SU................................1251

Bozoky, B................................ 1672

Brachtel, EF .............. 148, 177, 196

Bradely-Dunlop, D....................... 495

Bradley, D …………………...... 912

Bradley, G ................................. 1141

Bradley, KT .............................. 1164

Bradly, D .......................... 628, 649

Bradshaw, SH...…………….... 335

Braggio, E .................... 1163, 1278

Brambilla, E …………………... 1612

Brandt, SM ................................. 329

Brandwein, J ............................. 1247

Brat, DJ ........................ 1501, 1516

Bratman, D ............................... 1649

Braverman, AS ............................. 303

Braylan, RC............................... 1165

Brazelle, W................................ 1673

Braziel, R …………………....... 1190

Braziel, RM...................1174, 1258

Brennick, JB ............................. 1739

Brentani, H.................................. 550

Brentnall, TA...................613, 1400

Brethauer, U ................................... 670

Brezault, C ................................ 1452

Briaire, I .................................. 100

Briaire-de Bruijn, I......................... 75

Briceno, C ................................... 670

Bridge, J ……………………..... 1690

Bridge, JA …………....52, 89, 103

Brigl, A..................................... 1070

Brill, YM .........................136, 382

Brinton, LA ................................. 292

Broaddus, R............154, 1097, 1532

Brodsky, J..................................1111

Brodsky, S.................................. 1382

Brodsky, SV ..................1362, 1374

Brodsky, V.....................1299, 1351

Brody, JB ............................ 1475

Brogi, E ..........................326, 1767

Brolin, TJ ................................. 1385

Bronner, MN …………………... 716

Bronner, MP ...........613, 1400, 1469

Brooks, E................................. 1119

Brooks, JS ……………….... 45, 448

Brooks, MA............................. 1721

Broom, AD .................................... 157

Brousaides, N................1368, 1369

Broustas, CG ………………….... 208

Brown, H..................................... 4
Brown, IS .......................... 555, 556 Brown, JG …………….... 721, 85

Brown, K.................................. 1449

Brown, R ................................. 1492

Brown, RE...............571, 577, 1384

Browne, LW ............................ 1448

Browne, TJ ..................................22

Browning, D......................................... 160

Brownlee, NA …………....700, 844

Bruggeman, R ......................... 1426

Bruguera, M ............................ 1436

Bruins, HM .............................. 906

Brunelli, M........722, 723, 820, 821

Bruner, JM ………………....... 1492

Brunet, E ................................... 1357

Brunner, G................................ 458

Brunsveld, JP ................................. 1026

Brunt, EM …........ 1233, 1422, 1476

Brustmann, H ............................. 945

Bryant, R …………………….... 1250

Bryant, S ……………………... 920

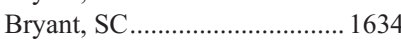

Brzostowski, E .............................326

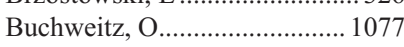

Buckingham, L......................... 1578

Buckley, PJ.......................501, 1207

Buckmeier, JA.............................549

Buckmiller, LM......................... 1136

Buckstein, R............................. 1197

Buczkowski, AK ……………... 1455

Buddingh, EP .................................. 37,75

Budnick, S............................... 1238

Budwit, DA .................................. 130

Buerger, H ........................254, 255

Bueso-Ramos, C ……………... 1185

Bueso-Ramos, CE ......... 1202, 1245

Bui, H............................................. 1618

Bui, HX ............................... 411, 544,

$693,863,1340$

Bui, M ............................471, 1673

Bui, MM..............40, 314, 518, 519

Bullock, MJ.............................. 1123

Bullon, A ……………………..... 487

Bundock, EA ..................................50

Bunnag, S................................... 1705

Buras, JA ..................1530

Burchette, J .................................1543

Burger, AM …………............ 1325

Burger, P..................................... 1484

Burger, PC .................................. 1497

Burgers, S.................................. 1646

Burke, A …………………..... 8, 336

Burke, B ....................... 688, 1114

Burkholder, KA............................ 1023

Burkholder, S ........................... 1765

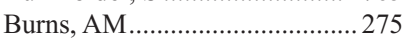

Burns, JM ............................... 1365

Burstein, DE................................ 956

Busaidy, NL................................539

Busam, KJ ................. 461, 481, 484

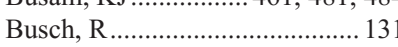

Busler, JF …………………....... 557

Bussolati, G.............................. 1722

Butler, SL …….................. 1165

Butrynski, JE....................... 76, 247

Butterly, L ................................... 653

Buza, N ..................................... 724

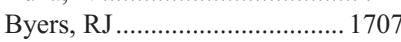

Byers, T.....................................197

Byler Dann, R ....................................... 1068

Byun, SJ ........................ 1499, 1509

\section{C}

Caballero, M .................. 1112, 1332
Cabay, RJ

Cabrera, RA...

Caceres, V …………………...... 168

Cacicedo, JM............................ 1524

Cagle, PT....................... 1580, 1581

Cai, G........356, 397, 750, 846, 928

Cai, T........................................ 841

Cai, YP ……………………...... 1565

Cain, MP …………………….... 884

Caines, JS ..................................... 275

Caire, FR ……………………... 1490

Cairo, MS ................................. 1268

Calabrese, F............................... 1588

Calin, G....................................... 675

Callard, P............................................. 109

Calonje, E................................... 457

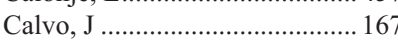

Calvo, KR ……................... 1239

Calvo, M ...................................... 660

Camacho, FI.............................. 1282

Camara-Lopes, LH.................... 1694

Cameron, DF ................................. 777

Cameron, JL ............................. 1396

Cameron, S.................................. 404

Camidge, R ………………....... 1617

Camilot, D.................................... 451

Camp, RL …………………...... 1667

Campan, M...................................292

Campana, F …………………......28

Campbell, J .................................. 623

Campbell, JA................................. 1445

Campbell, S............................... 1039

Campbell, SC ............ 879, 882, 891

Campo, E...................... 1160, 1167,

$1195,1265,1282$

Campos-Martin, Y......... 1157, 1226

Canales, M .............................. 1288

Cangiarella, J................................ 431

Cankovic, M _ _............... 1723

Cannizzaro, L ........................................ 1269

Cantley, LC …………………...... 642

Canto, M ............................... 1458

Cao, D ...... 725, 726, 771, 801, 946

Cao, JD.................................... 1176

Cao, QJ.......................................985

Cao, W …………...558, 1537

Capella, C.......................1306, 1417

Capelozzi, VL ………………... 1627

Capitanio, V ................................... 512

Capocelli, KE............................. 1166

Cappelli, L.................................. 772

Caprio, M .................................... 1686

Caramori, ML............................1378

Cardenas, VJ ............................... 1344

Cardesa, A ................................ 1112

Cardesa, T ……………………. 1167

Carey, LA ....................................235

Carini, M ........................... 747, 842

Carlin, A .................................... 1765

Carlo-Demovich, J ...................... 361

Carlotti, A.................................... 888,881

Carlotti, Jr, CG ......................... 1510

Carlson, AW …………………..... 1086

Carlson, DL ................... 1128, 1146

Carlson, G ..................................... 462

Carlson, JA........................ 455, 467

Carlson, JW ................................502

Carlson, MJ ........................................... 947

Carlstedt, T.................................95

Carmack, SW …………..... 559, 560

Carnero, A ............................... 1688

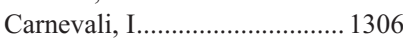

Carr, NJ ...................................... 556

Carrara, RCV ……………….... 1510

Carrasco, G …………………....... 561

Carrato, A …………………........... 546

Carretero, OA............................. 1385

Carter, $\mathrm{AB}$ …………………...... 1359

Carter, G.................................... 171

Carter, JE.................................. 1109

Carter, KM …………………..... 1728

Carturan, E _........................... 337

Carvalho, JC....................................... 911

Casadio, C................................ 1603

Cascio, MJ........................................ 41

Casey, MJ ................................. 988

Cashen, A …………………........ 1261

Cass, I.............................................. 1094

Cassarino, DS............................... 482

Cassivi, SD.................................. 1634

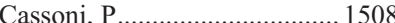

Castaneda, B …………………... 1352

Castanos-Velez, E …………….... 727

Castillejo, A................................... 546

Castillo, I.................................. 1283

Castillo, L.................................. 1343

Castillo-Martin, M ...................... 745

Catalkaya, A ........................... 1735

Catasus, L1......................... 948, 949

Cates, JM....................... 42, 88, 96

Cathomas, G.................................. 609

Cathro, HP.........................362, 728

Cavazzana, A............................... 747

Cawthorn, T …………………....... 1556

Ceballos, C............................... 1093

Celebi, J.......................................... 458

Centeno, BA.....................149, 1708

Cerda, S..............................669, 688

Cerutti, R................................... 1306

Cesarman, E ………………….. 1170

Cetin, N................ 845, 1168, 1169

Chadburn, A …............... 1170, 1324

Chaduvula, M............................... 1345

Chae, SH …………………......... 615

Chajut, A …………………….............. 1586

Chakraborty, S._.................... 1059

Cham, EM …………………….... 443

Chamberlain, WA....................... 1487

Chan, AT............................641, 642

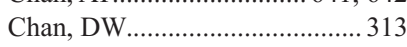

Chan, ES 1397

Chan, MM ……………............... 444,445

Chan, MP ................................... 445

Chan, R ..............................369, 977

Chan, RC.........................427, 1004

Chan, WC............ 1178, 1201, 1258

Chande, H ................................... 510

Chandler, KL ..............................132

Chandler, WM................................. 562

Chandra, P..................... 446, 1171

1326,1773

Chandran, UR ………………......517

Chandrasekhar, R ............215, 1609

Chang, A............................24, 1381

Chang, B ……………………...... 950

Chang, C .........................1272, 1303

Chang, CC............ 1183, 1271, 1584

Chang, C-C …………………..... 1753

Chang, H ............ 1172, 1223, 1229 ,

$1247,1275,1287,1713$ 
Chang, S-T ................................ 1181 Chang, TW …………………....... 135

Chang, Y...................................... 481

Changye, Z .................................. 81

Chapman, JA ................................ 133

Chapman, WC ……………….... 1422

Chapman-Fredricks, J ................. 731

Charbeneau, J........................... 1592, 1602

Chari, ST ................................ 1640

Charoenthammaraksa, S ........1576,

Chase, DR ……………………....2263

Chau, TK.................................... 1503

Chaudary, N ….......................... 1498

Chaunavel, AL ............................ 1490

Chaux, A................... 738, 739, 881

Check, I ...................................... 383

Cheetham, G .............................. 165

Chen, A...............................307, 319

Chen, C ………………... 300, 1172,

$1174,1190,1215$

Chen, C-H ................................ 133

Chen, D ..................................... 1175

Chen, DT ............................ 149, 250

Chen, E............................ 952, 1463

Chen, F ....................................... 134

Chen, G ..................................... 876

Chen, J........... 437, 454, 1362, 1713

Chen, K-C ............................. 1525

Chen, KM.................................... 137

Chen, L............. 354, 355, 638, 706,

$798,1169,1551,1687$

Chen, LM ..................... 1012, 1029

Chen, M....................................... 563

Chen, $\mathrm{PCH}$................................ 1173

Chen, R ........................... 613, 1400

Chen, W ......342, 1155, 1176, 1177 , $1193,1215,1248,1292$

Chen, WC....................... 135, 1173

Chen, X ...........................294, 778

Chen, Y.................... 128, 363, 764,

$765,1178,1194$

Chen, YB.................................... 1674

Chen, YL ........................................ 53

Chen, Y-T ........ 416, 623, 646, 1445

Chen, YY................... 156, 177, 664

Chen, $Z(G)$............................... 1139

Chenet-Monte, C.........................597

Chenevert, J.................................. 953

Cheney, RT .................................... 701

Cheng, H ................................... 1667

Cheng, L.......................... 722, 729 ,

$884,1180,1649$

Cheng, LL ................................ 196

Cheng, T.......................... 916, 1724

Cheng, Y...................................... 1503

Cheok, PY ................................. 310

Cherbavaz, D............................... 116

Chernock, RD ........................... 1120

Chernoff, K …………………....... 458

Chesney, A.................................... 1172

Chetty, R .......... 489, 520, 534, 588,

$1151,1152,1732$

Cheung, ANY ..................994, 1106

Cheung, DU ................................. 291

Cheung, M............................................ 1197

Cheville, JC......................721, 1086

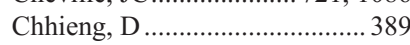

Chhieng, DC .............................. 104

Chiaravalli, AM................................... 1417

Chibbar, R

Chichareon, S............................ 1026

Chiem, F.................................. 1610

Childs, B ………………... 114, 115

Childs, TJ .................................... 960
Chiles, L........................... 515, 564

Chiles, LF ..................................... 136

Chilosi, M ................ 722, 820, 821

Chin, S.................................... 1062

Chinnaiyan, AM ............... 773, 774,

$824,826,853$

Chintagumpala, M..................... 1775

Chiong, E ……………………......... 707

Chiosea, S ............................... 1594

Chiosea, SI ................................ 527

Chiriboga, L .... 579, 719, 766, 1407

Chirieac, LR................. 1580, 1581,

$1582,1583,1635$,

$1670,1683,1689$

Chiselite, MD ........................... 1179

Chitale, D ...... 137, 333, 1513, 1723

Chitale, DA .............................. 1294

Chiu, A ……………………… 1180

Chiusa, L ....................... 1508, 1722

Chivukula, M .......... 138, 171, 1068

Chlenski, A................................ 1519

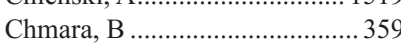

Chng, WJ... 1163, 1277, 1278, 1312

Cho, BM........................................ 604

Cho, ES ……………………..... 1493

Cho, H ....................................... 596

Cho, HY ....................................... 682

Cho, KJ ……………………....... 1130

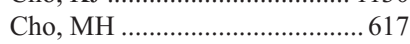

Cho, NY .......... 525, 648, 730, 1420

Cho, YM.......................... 802, 852

Choate, JD........................565, 658

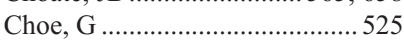

Choe, GY ................................ 1613

Choi, C ......................219, 616, 795

Choi, D ....................................... 600

Choi, DH ..................................... 632

Choi, J ..........993, 1020, 1085, 1421

Choi, JH ...................................... 586

Choi, KU ..................................... 603

Choi, PJ ................................. 1636

Choi, WL.................................. 1258

Choi, WW …………………....... 1201

Choi, Y ....................................... 139

Choi, YD ………………………..... 795

Choi, YJ ...... 377, 1001, 1081, 1133

Choppa, P........................ 126, 553

Chopra, S................................ 1724

Chou, AJ................................... 1571

Chou, CY …………………....... 1026

Chou, E .................................... 1524

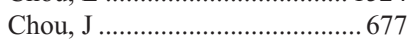

Chou, PM ……………... 1562, 1564

Choubey, D ........................ 693, 863

Cho-Vega, JH ........................... 1230

Chris, P..................................... 1347

Christal, JL ...............................1675

Chu, CK ……………...... 672, 1465

Chu, P.......................................263

Chu, PG ..................................... 1494

Chuang, C .................................. 1590

Chuang, S-S ............................. 1181

Chuang, Y-L ................................ 157

Chui, DHK ………………........ 1236

Chun, A …………………….... 1704

Chung, DH …………..... 1613, 1650

Chung, F................... 514, 515, 542

Chung, JH ...................... 1613, 1650

Chung, SW ................................ 1455

Cibas, ES .................................. 412,419 ,

1055,1738

Cibull, ML...............................2 274

Cieply, K ………..... 429, 611, 846,

Cimino, A........................ 140, 1722
Cin, PD.

1039

943

Cioffi-Lavina, M ......................... 731

Cipriani, NA................... 1591, 1592

Claret, FX............................... 1158

Clark, BZ................................. 1188

Clark, P................................... 394

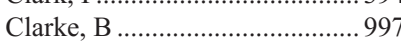

Clarke, BA.....................954, 1057

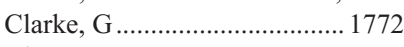

Clary, K ……………………....359

Clavel, C …………………... 1026

Clavero, O....... 738, 739, 974, 1026

Clayman, GL............................... 539

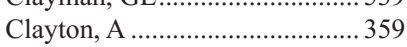

Clayton, AC.............................. 1743

Clayton, FC .......................................... 562

Cleary, TJ …….................... 1745

Cleaves, C .................................... 364

Clebanoff, JL................................ 732

Clement, PB ...............................994

Clement-Kruzel, S.......................955

Cleton-Jansen, AM.... 75, 100, 1565

Clevenger, JA............................... 733

Climent, F ………………...... 1167

Clingan, WJ.................................. 736

Clynes, M.................................212

Coban, I................ 672, 1392, 1398 ,

$1399,1443,1444,1465$

Cobleigh, MA...............................299

Coccia, PF ..................................... 1264

Coco, DP ......... 566, 567, 668, 1150

Codispoti, KT........................... 1182

Coffey, D .................................. 1771

Coffey, DM …………………..... 975

Coffey, RJ.................................... 692

Coffin, CM ............. 42, 88, 89, 1566

Cohen, C …............... 131, 150, 159,

$220,229,252$,

$378,462,570$

$787,1517,1766$

Cohen, CD................................ 1372

Cohen, D ......................1586, 1701

Cohen, HG …………............... 1331

Cohen, J...................................... 909

Cohen, JK …………………..... 1761

Cohen, JM ................................... 417

Cohen, L........................1630, 170

Cohen, ML ………………....... 1487

Cohen, P................724, 1652, 1658

Cohen, PR ……………………...956

Cohn, DE........................................ 947

Cohn, SL ............................. 1519

Colanta, AB …………………..... 1121

Colas, B ...................................... 810

Colby, TV ..................................... 1640

Colditz, G.................. 141, 142, 320

Cole, K ………………...... 141, 142

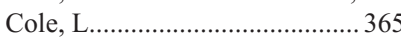

Colecchia, M ………………….... 734

Coleman, JF ............................... 1400

Collecchi, P ...........................747

Colli, BO …………........................ 1510

Collins, AB..................1363, 1364

1368,1369

Collins, L..........................141, 142 ,

$43,144,320$

Collins, LC .................................. 121

Collins, V .....................1339, 1348

Colomer, D................................. 1265

Colomo, L ………………….... 1160

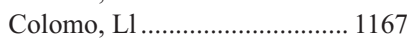

Colovai, A ................................ 1293

Colqhoun, SD............................... 514

Colvin, RB ................... 1363, 1364,

1368,1369

Comb, M ..................................1651

Comfere, N............................... 1308

Conklin, J ..................................564

Conlon, E ……………………..... 513

Conlon, K ………………....... 1179

Conlon, KP................................ 1681

Connelly, JH............................. 875

Conner, MG ………………....... 1095

Connolly, J ................................. 320

Connors, LH.................. 1200, 1777

Constinean, S ............................. 186

Conte, R …………………...... 1269

Contreras, A.........................145, 187,

$188,897,1122$

Conway, K............................... 235

Conzen, S ................................ 1642

Cook, C ................................... 765

Cook, JR.............. 1187, 1300, 1302

Cook, SL .................................1338

Cookingham, C ...................146, 147

Coon, J ………………........... 1578

Cooper, K.....366, 1119, 1144, 1653

Cope, W …………………..... 1123

Coppola, D …………….....314, 518,

$519,593,1406$

Corakci, A .................................. 1044

Corben, AD ………………...... 148

Corbishley, C................................ 715

Corcos, J........................... 742, 743

Cordoba, O.................................269

Coriat, R.................................. 1655

Corless, C.................................. 1256

Corley, L.......................... 575, 625

Cornell, LD …………... 1365, 1640

Cornish, TC ................................. 338

Corrales, M .................................... 1116

Correa, AM ………………....... 625

Cortina, G........................ 529, 1354

Corvalan, A …… …….......... 561

Cosar, EF ..................................... 390

Cosatto, E...…………………....245

Cosimi, AB .............................. 1363

Coskun, T ................................ 1735

Costantino, CL ……………...... 1475

Costello, LC …………………..... 744

Cota, AL ……………............ 571

Cotler, SJ .................................... 1405

Cotrell, A .................................... 1725

Coulter, DW .............................. 1264

Couturaud, B ................................. 488

Couturier, J................................... 488

Covas, DT …………………...... 1510

Covens, A ....................................995

Coviello-Malle, J............................ 735

Cowan, D ………………...235, 567

Cowher, MS ................................. 398

Cox, CE...................................... 176

Cox, M ....................................... 1366

Cox, RM...................736, 737, 856

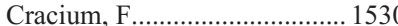

Cramer, HM .............. 415, 629, 680

Craver, RD ………………...... 1515

Crawford, B................... 1012, 1029

Crawford, ED ............................... 694

Cresswell, N ........................... 8, 336 
Crum, CP.................. 951, 952, 971, $972,1011,1021,1040$ $1055,1056,1067,1076$ $1111,1574,1575$

Cruz, L .................................. 1495
Cruz, LZ

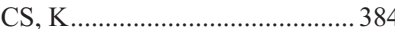

Cualing, HD .................... 485, 1337

Cuatrecasas, M........ 948, 949, 1042

Cubilla, AL ........ 738, 739, 848, 881

Cuff, J...........................957, 958

Cui, H..................................... 1237

Culhaci, N ............................... 1443

Culhane, AC ...................... 149, 250

Cullen, J ………………......764, 765

Cumbie, K........................ 429, 846

Cummings, OW ......................... 1649

Cun, L.............................624, 665

Curcio, F ................................. 1488

Curran, C.................................... 353

Currens, H ................................ 1665

Curry, C..................................1271, 1272

Curry, CV ................................. 1183

Cutlan, J ...................................... 468

Cuzick, J..................................... 861

Czader, M......................1180, 1676

Czerniak, BA.............741, 771, 806

Czerniecki, BJ ………………..... 194

Czernobilsky, B.......................... 1089

\section{D}

D Antonio, KB

894

Da Silva, M ............................. 597

Dabbs, DJ................ 122, 123, 138

$178,179,180,259,264$

Dacic, S......611, 1127, 1143, 1580,

$1581,1593,1594,1635$

Dadisman, CS ........................ 1579

Dafferner, A.................................. 89

Dahiya, M ..................460, 474, 503

Dahiya, S.................................... 766

Daignault, S................................ 873

Dailey, VL.................................... 740

Dal Canto, M............................ 84

Dal Cin, P............. 52, 84, 87, 375

dal Cin, P..................................... 412

Dal Cin, P.......... 1040, 1241, 1553

Dalenc, F .................................. 227

Dalla-Favera, R ............................. 1170

Daly, B .................................... 1638

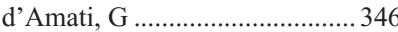

Damjanov, I............................... 265

d'Amore, ESG........................... 1562

D'Amore, KL .............................. 424

Dancer, JY ................................. 741

D'Angelica, M .............. 1389, 1391

D'Angelo, E ............................... 959

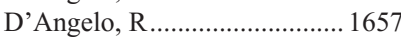

Daniel, HD .............................. 1467

Danielson, KG............................. 448

Dao, LD...................................... 1184

Daoud, N .......................... 164, 215

D'Arcy, CA ................................... 568

Dardick, I ..................... 1151, 1152

D'Armento, G ......................... 1722

Darnel, AD ....................... 742, 743

Darrow, MD ............................. 150

Dasari, RR ................................... 317

Dasgupta, A ................................ 325

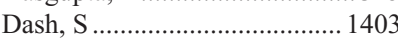

Dastane, AM …............................ 151

Daugaard, S................................. 98

Dausman, J.............................. 1535

Dave, BJ...

1178
Davidson, B............................... 674

Davidson, CM ............................ 960

Davidson, DD ............................ 1649

Davidson, N ............................... 114

Davidson, NE ............................ 115

Davies, R................................ 165

Davion, S..................................... 43

Davis, GJ......................................... 12

Davis, JS ..................................... 980

Davis, TL..................................... 452

Davis, WG.................................. 231

Davison, JM .............................. 592

Day, J......................................... 854

Daya, D .............. 1010, 1049, 1093

DCosta, A .................................. 1325

de Agustin, P .

de Biase, D ........................ 175, 277

De Brot, M ................................ 152

De Hertogh, G.......................... 1567

de Jong, D ................................... 75

De Jong, D .................................... 84

de Jong, D ...................................... 92

de Jong, DW............................. 1646

de Jong, J.......................................... 93

de la Taille, A ............................. 853

de las Morenas, A....................... 783

De Las Morenas, A................... 1114

de las Morenas, A.......... 1680, 1685

de Leval, L ................................. 1298

De Marzo, AM ............................ 772

De Nictolis, M.................................. 1038

de Noronha, SV ......... 339, 341, 343

De Peralta-Venturina, M ... 153, 430

de Peralta-Venturina, M .......... 1558

de San Jose, S.................. 738, 739

de Sanjose, S .................. 974, 1026

de Snoo, F ................................... 287

de Souza, A.................................. 1308

de Souza, W ............................ 1627

Deaton, R .................................... 1716

Deavers, M ................................. 983

Deavers, MT............. 99, 169, 1069

Debiec-Rychter, M....................... 84

deCastro, MG .............................. 705

DeCoteau, JF ............................... 1225

Deeds, J ....................... 1730, 1756

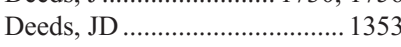

Deelder, AM ................................ 100

Degnim, AC .................................. 118

Dehner, LP ........................... 11, 991

deHoyos, AL ............................. 1628

Dei Tos, AP .................................. 661

Dejean, S .................................... 227

Del Carmen, M............................. 1084

Del Curto, B ............................. 1629

del Pino, M..................................... 974

Delabie, J...................... 1160, 1258

Delahunt, B ............................... 867

DeLair, D .................................... 961

Delattre, O.................................... 185

DeLellis, R ................................. 871

DeLellis, RA .........915, 1537, 1622

Delgado, C ................................. 1392

Della Barbera, M....................... 1588

Della Pelle, P................ 1038, 1369

Della Pelle, PA .............. 1364, 1368

Della Rocca, C ........................... 747

Delman, K ................................... 462

DeLuca, A ................................ 1645

DeMaglio, G ............ 163, 451, 1488

DeMartini, WB ........................ 106

DeMarzo, A.................... 895, 1545

DeMarzo, AM …....................... 814

DeMatteo, RP............................ 1389

Demetrick, D.............................. 1691
Demetris, AJ................. 1442, 1454 Demichelis, F ................. 781, 853, $885,1674,1677$

Dendrinos, K ............................... 669

Deng, A ............................. 466, 497

Deng, C ..................................... 1531

Deng, FM ................................. 1678

Deng, G........................... 624, 665

Deng, L.......................... 154, 1532

Denning, K........................ 513, 521

Denning, KD ........................... 1726

Denning, KM ........................... 511

Denton, KJ ................................. 962

DePalma, L................................. 1182

Dermen, A ...................................... 755

DeRoche, T ................................ 569

DeRosa, C .................................. 765

Desai, S ...................................... 339

Deschenes, JL............................... 155

Deshpande, C ............................ 650

Deshpande, CG ............ 1595, 1596

Deshpande, V ............. 78, 419, 640

$1148,1416,1429$

Desouki, MM .................... 174, 744

DeSouza, L................................. 918

Desphpande, V ............................... 408

Dessanti, P................................... 166

Devanath, A.............................. 570

Devaney, D................................ 1088

Devaraj, KM …........................... 745

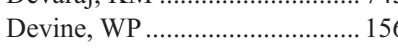

DeVore, S ................................... 1763

DeVore, SA............................... 1727

DeVries, S .................................... 51

Devries, S .................................. 128

DeWitt, JM................................. 629

Dewolf, W....................... 753, 767

Dexter, DF ........................................ 335

DeYoung, BR .................. 816, 1661

Deyrup, AT .................................... 44

Dhall, D.................. 514, 515, 542,

$564,1423,1424$

Dhall, G.................................... 514

Dhawan, MK ……..................... 1726

Dhesy, B ....................................... 160

Dhingra, S .................................. 571

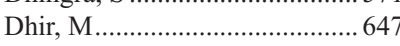

Dhir, R.................... 429, 746, 750

$782,846,928,1358$

Dhomen, N................................ 447

Di Carlo, V .................................. 512

Di Cristofano, C ............................ 747

Di Nicola, M ............................. 734

Diaz, A.................. 340, 1112, 1332

Diaz, JA......................................... 17

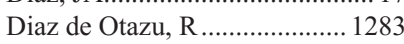

Diaz-Cano, SJ .......... 459, 516, 537

Diaz-Montes, TP ...................... 1052

Diaz-Perez, JA............................ 18

Dickson, BC ........................ 45, 448

Diebold, J ................................... 1263

Diem, M ......................... 111, 385

Dietel, M ............................... 260, 853

Diez Valle, R .............................. 1496

Difrancesco, L........................... 1691

DiGiuseppe, JA ........................ 1757

Dikshit, R ...................................... 82

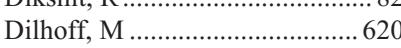

DiMaio, MA...................... 565, 658

Dimarino, AJ ............................ 1765

DiMichelle, A …..................... 235

Dimitropoulou, D...................... 1468

Dimov, N .................................. 1628

Dimov, ND ............................... 1185

Ding, P ........................................ 307,319

Ding, T ....................................... 1533

Ding, Y ........................................ 225

Dipaola, JE ................................. 526

Dirican, A .................................. 1402

Dirks, N................................... 1166

diSant'Agnese, A.......................... 323

Disanto, MG ............................... 382

Dishongh, KM................................. 748

Dispenzieri, A............................. 1184

Divine, G................................... 137

Diwan, AH ................................. 446

Diwan, $\mathrm{H}$................................... 480

Dixon, LR ............................... 1410

Djordjevic, B........... 955, 963, 964

Doan, N.................................... 1354

Doan, S................................... 1773

Dobi, A ................................. 764, 900

Dodd, LG .................................... 414

Doebele, R................................. 1617

Doell, H.................................... 1225

Dogan, A .... 350, 1163, 1210, 1242,

$1277,1278,1279,1307,1308$, $1317,1367,1706,1710$

Dogan, AH .................................. 440

Doglioni, C....................... 512, 821

Dogusoy, G ............................... 1402

Dokus, BJ .................................. 1755

Dolcet, X .................... 1042, 1075

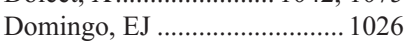

Dominguez, F............................. 362

Done, SJ ...................................... 362

Donev, K .................... 1489

Dong, HJ ................................... 1150

Donohue, K .............................. 1208

Donovan, JL............................ 1634

Donovan, MJ............................ 1682

Donovan, MN ................... 565, 658

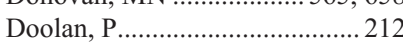

Dorfman, DM............................... 363

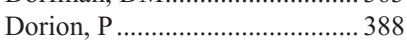

Dotan, Z …................................. 763

Dotti, G ..................................... 1272

Downs-Kelley, E ....................... 1679

Doyle, B .................................... 572

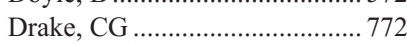

Drakos, E...................... 1158, 1186

Drakos, I................................... 1245

Drapkin, RI ................... 1021, 1022

Drauch, AJ................................. 1761

Dressler, L .................................... 235

Drew, PA .................................... 720

Drewnowska, K............................. 694

Dronca, RS .............................. 1222

Drury, SC .................................... 837

Dry, S ............................. 86, 1354

$\mathrm{Du}, \mathrm{J}$............................................ 1747

Duan, X................ 571, 577, 1492

Duarte, AGE...................... 717, 860

Dube, V .......

953, 965, 986, 995, 1043, 1046, 1047, 1058, 1071, 1072, 1091

Duboy, J .......

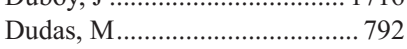

Dudas, ME ........................ 864, 865

Duffield, AS ............................... 749

Duffy, D ..................................... 867 
Dunn, ST ............................................ 1108

Dunning, KK .................................547

Duong, A .................................... 1523

Dupin, N …………………….....48

Dupree, W ………………….... 1025

Dupuis, SL ................................. 230

Dupuy, JF ..................................227

Durand, KA.................................... 1490

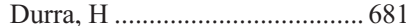

Dustin, S....................................... 987

Duvillard, P ................................... 631

Dvorakova, M ………………..... 750

Dyer, MJS

1279

\section{E}

Eastham, JA.................................. 892 Eberhart, CG ............................. 1497, 1526 Eble, JN................. 722, 820, 1649

Eby, PR ….................................. 106

Echeveste, J............................... 1496

Echeveste, JI .................................391

Edeline, J..................................... 751

Edgerton, ME................... 157, 1679

Edgerton, N …………………..... 1335

Edinger, JT ............................... 1188

Edmonston, T ............................ 1765

Edwards, RP............................ 1068

Edwards, WD ……………...2 27, 350

Egawa, S .................................. 1463

Egeler, RM .................................... 37

Egevad, L .................................... 812

Eggnener, S …………………..... 805

Egido, R ……………………....... 492

Eichinger, F .............................. 1372

Eickhoff, JC ………………..... 1769

Eiken, C................................... 1202

Eilber, F....................................... 86

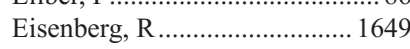

Eisengart, LJ ………………….... 752

El Hajj, H ................................. 1285

El Jamal, SM .............................158

El Mofty, SK ………………….... 1527

Elavathil, L...................... 160, 1049

Elavathil, LJ ................................. 161

Elbaruni, S........................ 970, 976

Eldibany, M............................. 1305

Eldin, K .......................... 1774, 1776

Elenitoba-Johnson, KSJ .......... 1179,

1252,1681

Elhassan, H …………………......516

Elit, L ........................................ 1093

Elkins, CT …………………..... 1124

Elliot, JN ...................................... 7

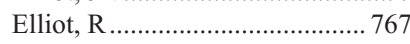

Elliot, WM …........................... 1645

Elliott, BE ……………………..... 133

Elliott, F ................................... 916

Elliott, R......................................753

Elliott, RM …………………...... 1491

Ellis, A........................................ 294

Ellis, IO .................... 162, 239, 246

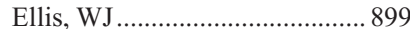

Ellison, K ................................. 1727

El-Mofty, SK ..................1113, 1120

El-Naggar, AK ......................... 1117

El-Salem, M …………………... 1189

Elsawaf, Z ………………....... 298

Else, T ……………………........ 1684

Elsheikh, SE ............................... 162

Elsheikh, TM....................................1121

El-Sibai, MF............................... 159

Elson, P ................................. 1214

Eltoum, I ..................................... 438

El-Zimaity, HMT ........................ 573
Emerson, RE ………………...... 415

Emery, LA .................................. 1680

Emmons, FN ................. 1293, 1321

Engelman, JA ................................ 642

Enriquez, EL …………………. 1696

Eom, DW …………………......595

Epstein, J ……………………..... 698

Epstein, JI.................. 699, 749, 756,

$799,800,813,814$

$829,831,833,835,843$, $849,889,895,898,904,923$

Erdamar, S............................... 1402

Erdogan, S.................................... 754

Eren, F ...................................... 966

Ereno, C ................................... 1118

Erenus, M................................. 966

Erickson, J J.............................. 548

Erickson, LA .................... 483, 1308

Erickson-Johnson, M .............. 1040

Erickson-Johnson, MR................. 69,

Erps, KA.................................. 507

Ersahin, C...................... 4, 266, 305

Ersahin, CH..................... 233, 1570

Escandon, J ................................ 811

Eshleman, J .............................. 1396

Eshleman, JR................. 1427, 1457

Espinosa, I................................... 289

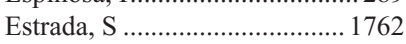

Etxezarraga, MC ....................... 1118

European Renal cDNA Bank

Consortium, ........................ 1372

Euscher, ED .... 933, 967, 968, 1741

Eusebi, V ……………………..... 277

Evans, A ........................................ 904

Evans, AJ................................. 1732

Evans, D …………………....... 1426

Evans, H........................................... 480

Evans, K.................................. 1713

Evans, MF ................................. 1119

Evason, KJ ................................ 969

Evdokimova, V .......................... 256

Evren, S.................................... 755

Ewanowich, CA ………………... 1007

Ewton, A......................1271, 1272

Ezzat, S ...................534, 954, 1498

\section{F}

Faber, J ................................... 1280 Facchetti, F................................. 1303

Fackler, MJ.................................219

Fadda, G.................................. 1102

Fadul, CE …………………...... 1719

Faerman, A ....................................... 763

Fagin, JA ......................... 531, 532

Fahey III, TJ .................................. 416

Fahim, S _......................... 155

Fahmy, MA ……………….......... 876

Fahy, J …………………........ 1720

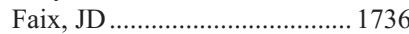

Fajardo, DA................ 804, 830, 834

Fajardo, DF ................................ 756

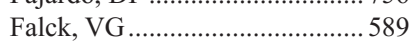

Falcon-Escobedo, R ………......257

Falconieri, G .......... 163, 451, 1488

Fall, K …………………............ 760

Falls, DG .....................................29

Falzarano, SM ………….....757, 758

Fan, C.................................... 164

Fan, C-Y.............619, 655, 737, 872,

$1125,1136,1153,1534$

Fan, F .............................265, 673

Fan, G.......................... 1174, 1190

Fan, X...................... 395, 425, 1518
Fan, XS

574,598

1503

Fanburg-Smith, JC ......... 46, 63, 67, $791,1336,1483$

Fang, X...................................... 575

Fang, Y ...................................... 842

Fanion, J ................................... 687

Faoro, L..... 1576, 1589, 1616, 1642

Faquin, W .................................523

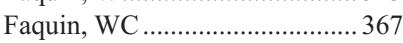

Farber, JL ................................... 1466

Farkas, DL …………………........ 1733

Farmen, SL................................ 1681

Farmer, P ....................................502

Farmer, PL...…………………..... 960

Farraye, F …………………....... 669

Farrell, M …….......................... 260

Farris, AB ............ 669, 1363, 1364

$1368,1369,1429$

Farshid, G.................................. 165

Fathallah, L ............................... 982

Faull, KF ......................................... 291

Faye-Petersen, OM ……………..... 33

Fazio, V ............................. 569, 656

Fazuoli, MG ................................716

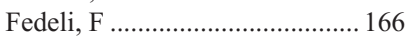

Fedor, H ..................................... 895

Feher, SA.................................. 1755

Feilotter, H ................. 133, 437, 454

Feld, MS................................... 317

Feldman, A ....................................... 1278

Feldman, AL.................1210, 1308

1317,1640

Feldman, MD

$.1137,1744$

Felgar, RE ……………... 1159, 1187

Feltmate, CM ............................. 971

Fend, F ………...... 590, 1298, 1313

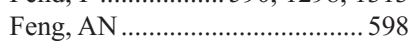

Feng, B ................ 1191, 1192, 1246

Feng, JN ..................................... 982

Feng, W........................... 576, 577,

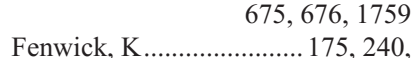

$255,277,1506$

Feratovic, R............................... 371

Feretzaki, M ........................... 1158

Fermin, D ..................... 1179, 1681

Fernandez, C ............................ 1672

Fernandez, E …………………........ 487

Fernandez, G............................. 1682

Fernandez, M ............... 1202, 1245

Fernandez, MP ............................ 452

Fernandez, PL ............................. 167

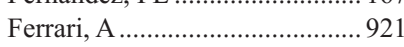

Ferreccio, C................................. 561

Ferreira, U ....................................... 717

Ferrell, L....................... 1418, 1476

Ferrell, LD................................ 1411

Ferrera, A........................................ 1026

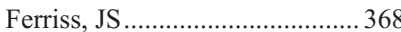

Ferro, P........................................ 166

Ferrone, CR .............................. 1416

Ferrone, S S................................... 728

Ferruelo, A................................... 810

Ferry, JA........................ 1090, 1148

Fetni, R.

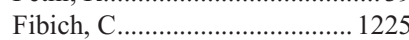

Ficarra, M...................................... 1403

Fiel, MI .................................. 1464

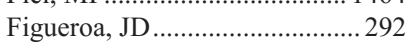

Filleron, T................................. 1692

Findeis-Hosey, J ......................... 1598

Fine, SW..................696, 759, 792,

$864,865,892,1752$

Fineberg, S ............................... 112

Fink, D ..................................... 1547

Finkelstein, A ….................464, 724

Finkelstein, SD.............. 1561, 1726

Finn, AJ ……………………...... 453

Finn, S ……………….... 513, 528

Finn, WG................................. 1728

Finzi, G …………………….... 1417

Fiore, C ……………….... 760,902

Fiorentino, M ....................760, 902

Fioretto, P............................... 1378

Firchau, DJ .....................................27

Fire, AZ ……………………... 1671

Firestein, R ...................... 578, 1683

Firestone, B .............................. 1686

Fisher, C .......................................... 44

Fisher, G.................................... 861

Fishman, E .............................. 1456

Fiske, WH …_........................ 692

Fitting, P.................................. 1154

Fitzmaurice, M............................ 317

Flanagan, AM......................... 55, 95

Flanagan, MB........................... 1126

Flanigan, R ................................ 770

Flavin, R........... 358, 513, 521, 976

Flavin, RJ ......................... 511, 970

Flejou, J-F …………………….... 631

Fleshner, N .................................. 755

Fletcher, CD .................. 87, 98, 840

Fletcher, CDM .... 50, 77, 100, 1553

Fletcher, S ........................ 143, 144

Fliedner, SF .............................. 1779

Flood, TA …………... 761, 762, 857

Flores, LM................................. 1738

Florman, S............................... 1403

Flotte, TF.................................... 440

Flotte, TJ ..................................... 483

Fogarasi, SI ............................... 1682

Fogo, AB ............. 1361, 1383, 1385

Foley, T ................................... 275

Folkins, AK ............... 951, 971, 972,

1022,176

Folpe, AL …............ 35, 38, 60, 69,

$70,71,89,721$

Fong, D .................................... 1177

Fonseca, R.......... 1163, 1278, 1367

Fontaine, D............................... 1007

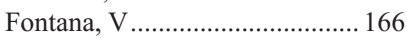

Foo, W..................................... 502

Foran, DJ ……………………..... 342

Forauer, AR …………………….... 405

Forcione, B................................. 1749

Fordjour, P............................... 1756

Forman, K ……………………..... 20

Formenti, S................................. 1702

Forti, G.................................... 842

Foss, RD....................................... 67

Foster, CS ............................................ 861

Foster, RS ……………………..... 733

Foucar, K................................... 1218

Foulkes, WD ...............................2 239

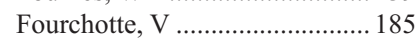

Fournet, JC ................................... 39

Fowler, D ...................................... 8

Fowlkes, N .............................. 1345

Fox, E.......................................... 614

Fox, KR ……………………...... 194 
Franklin, RB................................ 744 Franklin, W ………………….... 1617

Franks, TJ................................. 1619

Franzi, F ................................ 1417

Fraser, R …………………… 1563

Frater, J.................................... 1261

Frater, JL .................................. 1233

Free, A.................................... 1703

Freedland, S .............................. 727

Freedland, SJ ............................. 1543

Freedman, S ............................... 694

Freeman, S ………….... 1339, 1348

Fregnani, JH................................. 550

Freitas, LLL ………... 716, 717, 860

French, S ................................ 1432

Freneaux, P............................... 185

Fridman, E.................................. 763

Friedrich, V …..................... 1547

Frierson, HF ......................362, 728

Fries, RC …………………....... 386

Frishberg, D .............. 395, 425, 465

Frishberg, DP ............................... 515

Fritzler, MJ .............................. 1377

Fritzsche, FR .............................. 853

Frosina, D.................................. 461

Frost, M.................... 118, 288, 316

Frost, MH .................................... 251

Frugier, H ................................. 125

Fu, K .................. 1178, 1201, 1258

Fu, Y.......... 505, 753, 767, 768, 910

Fu, YX.................................. 1644

Fuchs, CS …………….... 578, 638

$641,642,643$

Fuda, F ………………….... 1310

Fuda, FS ……………………........ 1193

Fujii, T............................................ 1599

Fujita, H ……………………... 1328

Fujita, T......................................... 901

Fujiwara, M....................... 822, 823

Fukatsu, A ..................................901

Fukayama, M ..................630, 1030

Fukumura, Y.............................. 1404

Fukunaga, M ...............................973

Fukuoka, J. 1606, 1689, 1697, 1729

Fukusato, T.................................... 61

Fulford, LG ………………….....255

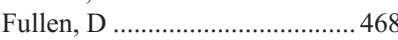

Fuller, C ……….........922, 1712

Fuller, CE …………………........ 1678

Fuller, GN …………………..... 1492

Fumagalli, C............................. 1629

Fung, P ....................................... 732

Fung, PC ……………………..... 362

Furlan, D …………………….... 1306

Furman, R …………………... 1324

Furth, EE .................................... 1434

Furukawa, T ............................ 1463

Furusato, B ............... 764, 765, 900

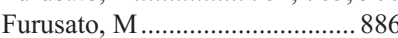

Fuste, V …….............................. 974

Fyfe, BS ...................................... 342

\section{G}

Gaafar, A ............................... 1118

Gabril, M..................................... 918

Gadre, SA................................... 169

Gafa, R .................................... 580

Gaile, DP................................... 174

Galateau-Salle, F..................... 1600

Galbincea, J.............................. 1773

Gale, J ..................................... 1196

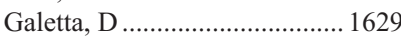

Galfione, SK .............................. 975

Galgano, MT .................... 170, 368
Galindo, L ................................ 317 Gallagher, M ................... 970, 976

Gallagher, SL ......................... 1755

Gallardo, A ............. 948, 949, 1042

Galli, S ............... 809, 1568, 1569

Galliano, G.................... 515, 1733

Galliano, GE ............369, 427, 977

Gallina, G................................... 661

Gallinger, S ............................. 1453

Gallo, P..................................... 346

Galvin, JR ............................... 1619

Gamez, JD.............350, 440, 1307 ,

$1367,1706,1710$

Gamez, R.................................... 131

Gamez, RG.......................... 9, 370

Gandhi, A ............................. 1657

Gandhi, M ............................ 517, 541

Ganesan, S.................................. 407

Ganguly, A................... 1039, 1148

Ganjei-Azar, P....................... 1104

Gao, F..................................... 171

Gao, J .............................. 172, 1194

Gao, JX ................................... 1687

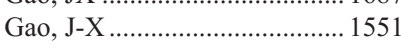

Gao, W ...................................... 817

Gao, Y ........................ 1353, 1730

Gao, Z .................................... 1442

Gao, ZH................................... 589

Garabedian, MJ .......................... 921

Garady, CI ................................... 454

Garberoglio, CA.......................... 263

Garces, JC ................................ 1330

Garces, YI ................................ 1634

Garcia, A ....................... 670, 1195

Garcia, B ................................... 660

Garcia, C ................................. 1196

Garcia, F....................... 1112, 1332

Garcia, FU......................... 124, 778

Garcia, H.......... 811

Garcia, J .......................... 1390, 1461

Garcia, JF .................... 1282, 1288

Garcia, JJ................... 1127, 1128

Garcia, MT .................. 1104, 1393

Garcia, R ................................... 1155

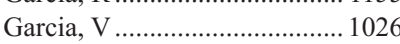

Garcia-Albeniz, X ..................... 855

Garcia-Closas, M ......................... 292

Garcia-Diaz, JB ........................ 1330

Garcia-Fernandez, E ................ 1038

Garcia-Manero, G .................... 1171

Garcia-Moliner, M .................. 1611

Garcia-Pagan, JC....................... 1436

Gardner, H................................ 1538

Gardner, TB.................................... 612

Gardner, WA............................. 1541

Garg, K...................371, 978, 1087

Garica, RL................................. 935

Garijo, MF................................. 105

Garland, S ................................. 1026

Garmendia, M .......................... 1118

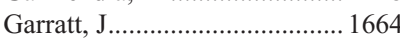

Garrett, CT ............................... 1693

Garrison, R................................. 394

Garrity, E...................... 1591, 1605

Garver, J .................................... 390

Garvin, AJ ........................ 406, 700

Gashinsky, I.............................. 1323

Gaspa, A................................... 855

Gatalica, Z.... 173, 563, 1531, 1690

Gatell, JM............................... 1332

Gatter, KC ................................ 1544

Gattuso, P..... 199, 299, 1341, 1578

Gaudet, MM............................... 292

Gaulard, P................................ 1195

Gaut, J ....................................... 1369
Gavino, AV .................................. 192

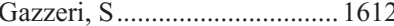

Gebhardt, MC .............................. 73

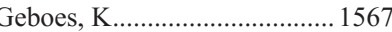

Geddie, WR............................ 1141

Gee, JM ..................................... 162

Geisinger, K ............................ 1596

Geisinger, KF ................................ 557

Geisinger, KR.................. 406, 557

Gelabert, L ................................ 167

Gellert, L ................................... 103

Gellert, LL.................................. 766

Gemmer, J ............................. 1673

Genega, EM ................ 505, 753, 7

$67,768,910$

Genevay, M................... 640, 1416

Geng, B ...................................... 1233

Genta, RM....................... 559, 560

Geoghegan, J........................... 1659

George, TI .............................. 1256

Geradts, J.................................. 174

Geraghty, R ........... 581, 614, 1659

Gerald, W .......................666, 706

1595,1596

Gerhard, GS .......................... 870

Gerney, G ...................... 529, 1354

Gerstein, MB............................ 1677

Gerszten, E.............................. 1327

Geskin, LJ ................................. 1188

Getzenberg, RH.......................... 894

Geyer, F....................................... 254

Geyer, FC ................ 175, 277, 447

Geza, A....................................... 332

Ghali, V ...................................... 599

Ghayouri, M.......... 176, 318, 1708

Ghazarian, D ................. 489, 1714

Ghazarian, MD........................... 954

Ghazizadeh, M .............................. 1778

Ghebremichael, M..................... 768

Ghirardi, G .............................. 990

Ghorab, Z ............... 965, 986, 995

1043, 1046, 1047, $1058,1071,1072,1197$

Ghossein, R ....................... 531, 532

Giannakoulas, N........................ 1297

Giannini, C............................... 1514

Gibory, H..................................... 1701

Gibson, JA........................ 582, 583

Gibson, LE ................................. 450

Gilbert, CM ................................ 1731

Gilbert, R.................................. 1135

Gilbertson, J ............................ 135

Gilbertson, JR …........... 1299, 1360

Gilbrech, L ................................ 655

Gilcrease, MZ ….............. 231, 257

Gilks, B ..................................... 961

Gilks, BC................................. 1664

Gilks, CB...............932, 934, 979

$1007,1008,1019,1057$

Gill, JR ...................................... 28

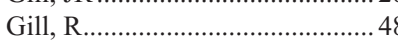

Gill, RM .............................. 177

Gillan, JF ................................. 1720

Gingell, DC .............. 178, 179, 180

Giordano, C.............................. 346

Giordano, T ............................. 528

Giordano, TJ........ 1648, 1668, 1684

Giorgadze, T.......................... 938

Giorgadze, TA …........................ 252

Giostra, E ................................... 1433,1460

Giovannucci, EL ............. 578, 641,

642,643

Giri, N ..................................... 1254

Giusto, D ........................ 628, 649

Gjertson, D.............................. 1432

Glas, AM ..................................... 287

Glasker, S .................................. 941

Glass, LF .................................... 485

Glazyrin, A ................................. 455

Glennie, C ............................. 1707

Gluck, S...................................... 283

Gobbi, H........................... 152, 280

Gobbo, S ......... 722, 723, 820, 821

Godambe, AS ................ 233, 1570

Godleski, JJ ............................. 1601

Goel, S............................. 10, 1109

Goes, NB.................................. 1363

Goez, EI ................................. 1116

Gofstein, S............................... 1758

Goggins, M ..................596, 1396,

1457,1458

Gogos, C ................................ 1468

Gokden, M ............................ 856

Gokden, N............. 736, 737, 748

$822,823,856$,

$859,872,1366,1780$

Gokhale, S............................... 1535

Goksel, S .................................. 1402

Gold, MA ................................... 1108

Goldberg, J ................................. 326

Goldblum, JR ................... 94, 493,

$569,601,656$

Goldsmith, JD ................... 73, 635

Goldstein, DP ............................. 972

Goldstein, LC.................. 290, 1296

Goldstein, LJ ..................... 114, 115

Goldstein, NS .................. 704, 825

Goldstein, S............................. 1774

Goldthwaite, P......................... 1497

Gollin, SM................................ 1187

Gomes, AM ............................... 1361

Gomez-Fernandez, C ........ 279, 731

Gonatas, NK........................... 1502

Gondra, MM ............................ 980

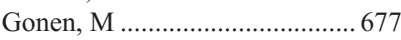

Gong, GY ................................... 852

Gong, M ................................. 758

Gong, MC .................................. 882

Gong, Y .................... 181, 191, 372

Gonlusen, G .............................. 754

Gonsalves, N ............................ 651

Gonzalez, A.................................... 530

Gonzalez, JL............ 405, 984, 1092

Gonzalez, M.............................. 660

Gonzalez-Abraldes, J ............... 1436

Gonzalez-Barca, E ................... 1167

Gonzalez-Berjon, JM ................... 769

Gonzalgo, M ............. 699, 803, 908

Goodarzi, M .............................. 584

Goodgame, B ........................... 1597

Goodman, MA............................... 79

Gopal, P..................................... 373

Gopalan, A..............696, 759, 792

$864,865,892,1752$

Gopinath, A …............................ 1667

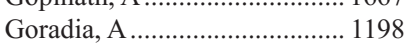

Goranson, E................................... 16

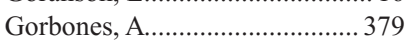

Gorbonos, A ..................... 702, 770

Gordon, IO ............................... 1602

Gordon, $\mathrm{N}$................................... 16 
Govindin, R............................. 1597 Gown, AM...............290, 705, 1296 Gradowski, JF ......................... 1199 Graf, HP ......................................245

Graham, AR ………………….....507

Graham, DY ………………….... 573

Graham, K................................ 1685

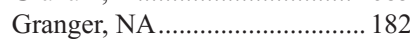

Granja, NM ……........................ 129

Gratzinger, D............................ 1249

Gray, R ........................... 114, 115

Grce, M ................................... 1026

Greaves, W...………………..... 1622

Greco, MA.................................... 719

Green, AR ..................................... 162,239

Green, P........................... 382, 678

Green, R ..................................276

Greenaway, J ............................. 1704

Greenberg, JA.............................. 669

Greene, MJ ................................. 1200

Greenfield, L …………..... 181, 372

Greffe, B................................. 1166

Gregoire, J.................................953

Greiner, TC …............... 1201, 1258

Grenert, JP................................ 1411

Grier, DD ................................. 1165

Griffin, C ......................................204

Griffin, KA ................................ 1373

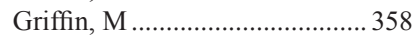

Griffiths, D .................................. 812

Griggs, J .................................... 311

Grignon, DJ.................................. 807

Grigoriadis, A................... 244, 255

Grigsby, PW .............................. 1113

Grimer, RJ .................................... 90

Grimm, EE …………………....... 183

Grin, A................................... 1047

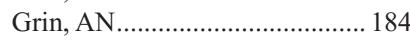

Grinkemeyer, M ......................... 777

Grisak, D.................................. 1224

Grisanzio, C ................................ 768

Grogan, T .................................. 260

Grogan, TM.............................. 1300

Grogg, KL ..........................38, 1210

Groisman, GM ............................ 585

Grondin, K …………………...... 953

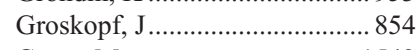

Gross, M................................... 1543

Grossman, DM............................. 294

Grosso, L..................................... 1295

Growdon, WB ……………….... 1009

Gru, AA ………………....... 11, 981

Grudzinska, M............................ 344

Gruel, N..................................... 185

Gruidl, M........................... 149, 250

Grzybicki, DM .......................... 1660

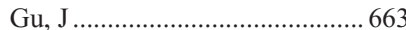

Gu, K........................................ 1201

$\mathrm{Gu}, \mathrm{M}$....................................... 1724

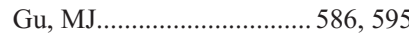

$\mathrm{Gu}, \mathrm{T}$.................................................. 1651

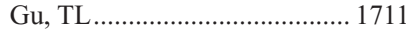

Gualco, G .................................... 153

Guan, H................... 936, 939, 982

Guarner, J ......................1333, 1347

Guenther, S................................. 521

Guenther, SM ................................511

Guerrero, L..................................... 1519

Guggisberg, KA ........................ 1129

Gugliotta, P ..................................... 240

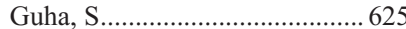

Gui, D..............................529, 1354

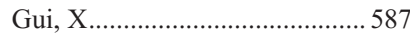

Guillaudeau, AN ……………... 1490

Guillen, C....................................546
Guillen, S ............................... 1327

Guillerman, P ........................... 1775

Guillonneau, B ………………..... 892

Guimera, N............................... 1026

Guindi, M..................... 1453, 1732

Gulbahce, HE............ 131, 370, 413

Guler, G..................................... 186

Gullane, P................................... 1135

Gullett, AE …………………... 1492

Gumurdulu, D ......................... 754

Gunn, SR ................ 328, 1696, 1751

Guo, C ................................... 707

Guo, CC ……..... 741, 771, 806, 898

Guo, M ....................................... 983

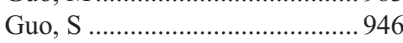

Guoit, MC …………………….... 876

Gupta, A …………….............. 1477

Gupta, G................................. 1493

Gupta, M ..............984, 1279, 1740

Gupta, P................................. 1208

Gupta, R ................. 395, 425, 703,

705, 711, 712, 721, $819,840,851,1603$ $1604,1620,1621$

Gurel, B ...................................... 772

Gurevich, I ................... 1202, 1203

Gurka, KK ..................................362

Gurkar, AU ............................... 1536

Gurpide, A..................................... 391

Gustafson, SA .......................... 1204

Gutierrez, JA ...................................... 1513

Gutierrez-Garcia, G.................. 1167

Guzman, G .................................. 1405

Gwin, K.................. 145, 187, 1098

Gwozd, C ............................... 1608

Gyure, KA................................... 1487

\section{$\mathbf{H}$}

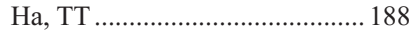

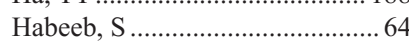

Habel, L............................ 143, 144

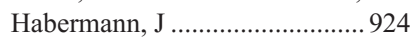

Habib, C ...................................... 985

Hadengue, A................................ 1460

Hafez, M .................................. 1037

Hafez, N ……………………....... 519

Hafez, NA ................................... 1406

Hafezi, S................... 409, 588, 986

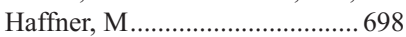

Hagan, S..................................... 572

Hagemeister, F ........................... 1271

Hagenkord, JM................. 875, 1355

Hahn, HP...........................582, 583

Hahn, WC..........................578, 1683

Hahn, YS .................................. 1401

Hajdu, C ................................................... 579

Hajdu, CH ...................... 719, 1407

Hajibashi, S................................... 64

Hakam, A......................... 518, 1406

Halai, DD ...................................... 55

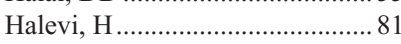

Hall, R ............................................ 382

Hall, SJ ........................................... 797

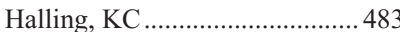

Hallmark, KC ................................. 1656

Halloran, PF ............................. 1705

Halushka, M.................... 140, 1545

Halushka, MK .....................306, 338

Hamazaki, S ………………..... 1286

Hameed, M.............................. 189, 924, 1311

Hameed, MR …………………... 1493

Hameed, O .................104, 190, 278 ,

$304,321,710,740$

Hamele-Bena, D.............. 745, 1485
Hamilton, CR ……………….... 1771

Hamilton, LE..................................58

Hamilton, SR...................... 549, 575

Hammer, G.............................. 1684

Hammock, BD .............................637 637

Hampel, H.......................... 689, 697

Hamstra, DA ………………….... 824

Han, B ............... 773, 774, 824, 853

Han, CW...................................219

Han, EY........................................ 191

Han, JS ............................. 192, 193

Han, R ………………….... 466, 497

Han, X .................................. 1245

Handy, BC ................................. 1204

Haney, J...................................... 1617

Hanley, K ................................... 987

Hann von Weyhern, C.................. 590

Hanna, W .................. 160, 262, 334

Hanna, WM ……………………....267

Hannah, C …………………….... 626

Hannigan, C ................................ 358

Hano, H...................................... 886

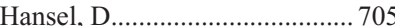

Hansel, DE ....................... 775, 776,

$879,882,891$

Hanson, CA.................. 1162, 1175 ,

1222,1240

Hantschke, M ................................ 457

Hao, L............................ 1652, 1658

Hao, LH.................................... 1024

Hao, S............................1243, 1494

Hao, SY ................................... 1542

Haque, R ..............................143, 144

Haque, S.................................. 1403

Harada, S.................................... 194

Haralambieva, E.......................... 1205

Hardesty, J................................. 988

Hardisson, D .......... 255, 1034, 1038

Harfe, BD .

..... 42

Hari, P .............................................. 1746

Harlow, S.....................................230

Harocopos, GJ ............................ 1527

Harpaz, N .................................... 587

Harper, T …………………….... 1716

Harrington, A....................................... 1728

Harrington, AM ………………... 1206

Harris, NL ............................... 1090, 1148

Harshan, M.................................. 417

Hart, J...................... 644, 645, 684, $685,686,1428$

Hartman, AK .............................. 12

Hartmann, A ................................. 727

Hartmann, D ....................................... 1520

Hartmann, E ............................. 1205

Hartmann, L ............................... 288, 316

Hartmann, LC .................... 118, 251

Hasegawa, M.............................. 203

Hasegawa, T.................................. 74

Hashimoto, H ............................... 49

Hassan, A....................... 1233, 1261

Hassanein, NM........................... 1207

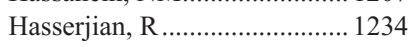

Hasserjian, RP .......................... 1208

Hastie, T ................................... 1669

Hatcher, D ……………………........... 766

Hatcher, L.......................... 173, 563

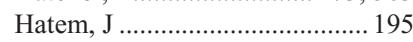

Hatfield, S .............................. 1530

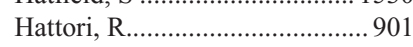

Haubein, N ............................... 1762

Hault, K..................................... 341

Hauss-Wegrzyniak, B..................260

Havens, MA …………………...... 374

Hawk, A........................................ 1755

Hawksworth, D ............................ 764

Hawthorn, LA ……………….... 1698

Hayashi, R.............................. 1606

Hayashi, T ............. 657, 1010, 1404

Haydt, L ................................ 1114

Hayek, K ........... 288, 936, 939, 982

Hayem, C …………………….... 281

Hayner-Buchan, A...................... 1212

Haynes, CM ………………….... 182

Hayran, M ………………….... 186

Hayward, R …………………….... 447

He, D .......................... 1353, 1686

He, G ..................... 83, 1551, 1687

He, J …………………….............. 291

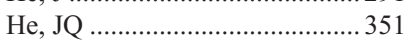

He, M ………………... 1571, 1633

Hebert, L .................................. 1362

Hecht, JL _........................... 1076

Hedley, DW............................... 1141

Hedvat, C ......................... 327, 1080

Heenan, PJ................................... 470

Heerema-McKenney, A................735

Heffron, CC................................ 970

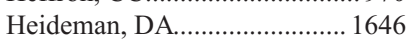

Heiden, T................................. 1209

Heidenberg, DJ.............................. 777

Heider, A ……………………….... 989

Heintzelman, RC .......................... 778

Heitz, PU ...................................... 533

Helenius, G ……………………... 885

Heller, DS......................1066, 1572

Helm, J .................... 518, 519, 1708

Henderson, E............................ 1708

Henderson, VM ………………..... 836

Henderson-Jackson, EB ............518,

519,593

Hendrickson, MR ….................. 1028

Hengel, K ........................................... 303

Henger, A ................................... 1372

Hennessy, B.......................154, 1532

Henriksen, KJ............................ 1605

Henry-Tillman, R ....................... 158

Hensbergen, PJ...……………..... 100

Hensler, AM ……………............ 928

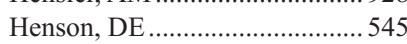

HER2 Group ………………....... 168

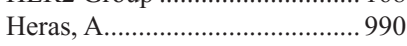

Herawi, M ................................... 813

Herberger, SD …………………. 196

Herceg, ME ................................... 96

Herebert, H................................. 1651

Heretsch, S ...................................... 57

Herman, J ................................. 1456

Hernandez, AV .......................... 757, 818

Hernandez, J............................. 1557

Hernandez, VS ............................... 80

Hernandez-Cortes, G................. 1034

Hernandez-Losa, J...................... 1688

Hernandez-Rodriguez, J............. 349

Hero, AO …………………....... 1728

Herrera, GA...................... 1370, 1371

Herrera, LP................... 17, 18, 779,

780,1393

Herreros, B .............................. 1283

Herrick, JL .................................. 1210

Herrmann, FR ………………....... 701 


\begin{tabular}{|c|c|}
\hline Hicks, DG ...................... & Hossain, D.................................. 1548 \\
\hline $307,311,319$ & Hossain, R. \\
\hline$. .772,844,1545$, & Hotchkiss, RS..... \\
\hline $1774,1775,1776$ & Hou, N.............. \\
\hline$\ldots \ldots 822,823,957$ & Houk, L ........................... \\
\hline Higgins, RA............................... 1675 & Houser, PM ....................... \\
\hline Hill, GA................................ 863 & Howanitz, JH............................ 1323 \\
\hline Hill, ID .......... & Howanitz, PJ ................ \\
\hline 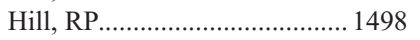 & 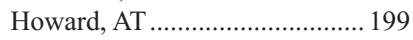 \\
\hline ............... 347 & Howard, L ...... \\
\hline Hilliard, NJ...... & Howard, LM............................. 1725 \\
\hline Himmetoglu Ussakli, C............. 186 & Howard, MT................. 1213, 1214 \\
\hline 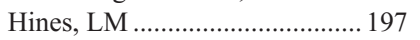 & Howe, SL ......... \\
\hline …........... 55, 95 & Howell, R ......... \\
\hline …................... 630 & Hoyer, JD ........... 1162, 1184, 1240 \\
\hline Hinson, $\mathrm{J}$........ & Hoyland, JA................................ 1707 \\
\hline$\ldots 651$ & 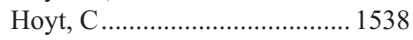 \\
\hline Hirsch, MS. & Hoyt, KM.. \\
\hline 952,97 & Hruban, R.... \\
\hline$\ldots 155$ & Hruban, RH... \\
\hline 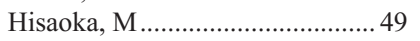 & $1458,1463,1475$ \\
\hline Ho, LC.......................................... 1211 & $.1213,1214,1284$ \\
\hline Ho, SM...... & Hsiao, C-H ........ \\
\hline Hoang, MP .... & Hsieh, P-P................................ 1181 \\
\hline Hochberg, E.. & $\mathrm{Hsu}, \mathrm{CH} . . . .$. \\
\hline Hochberg, EP. & Hsu, M-Y .......... \\
\hline Hocker, K....... & 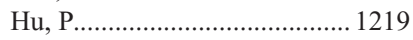 \\
\hline Hoda, SA.......... & ....... 141, 142,320 \\
\hline Hodgin, JB ….............................. 1372 & $\mathrm{Hu}, \mathrm{Y} \ldots \ldots \ldots \ldots$ \\
\hline Hodul, P ......... & Huang, AY.... \\
\hline 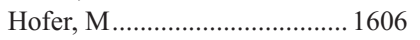 & 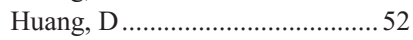 \\
\hline .....781, 854, 1677, & Huang, G........... 1163, 1277, 1278 \\
\hline $1689,1697,1729$ & Huang, H..... \\
\hline$\ldots \ldots \ldots \ldots \ldots \ldots \ldots . . .349$ & Huang, HY .. \\
\hline Hofstetter, WL. & Huang, H-Y... \\
\hline Hogarth, DK.............................. 1602 & Huang, J .........................232, 499, \\
\hline Hogarth, M.... & $1174,1190,1598$ \\
\hline Hogendoorn, PC................... 97, 98 & Huang, MA....... \\
\hline Hogendoorn, PCW …....... 37, 75, & Huang, Q ...............574, 598, 668, \\
\hline $100,101,1565$ & Huang, S............ \\
\hline 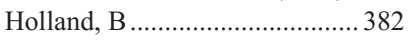 & …........ 784, 858, \\
\hline Holland, SK..... & $1032,1303,1769$ \\
\hline Holmang, $\mathrm{S}$... & …............... 1183 \\
\hline Holt, GE ....................... 42, 88, 96 & Huang, W-T................. 1181, 1753 \\
\hline Homan, SM..... & Huang, WW ..... \\
\hline$\ldots \ldots \ldots \ldots \ldots . . .42,88$ & 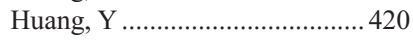 \\
\hline Honebrink, TJ................................ 13 & Hudacko, RM........................... 342 \\
\hline Hong, J ..................................... 1537 & Huebner, K ......... \\
\hline 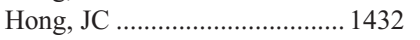 & 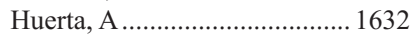 \\
\hline Hong, S-M..............595, 596, 1457 & Huettner, P........ \\
\hline 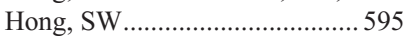 & Huettner, PC ..................991, 1006 \\
\hline Hong, YO .................................. 1130 & Hughes, A......................... 19, 1446 \\
\hline Hooda, S................................... 782 & Hughes, R.......... \\
\hline Hoofnagle, JH .......................... 1430 & Hughson, M....... \\
\hline 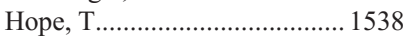 & Huh, GY ................................. 615 \\
\hline 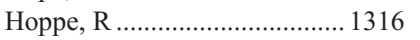 & 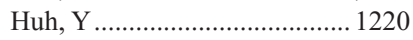 \\
\hline Horbinski, C.. & Huh, YO ........ \\
\hline 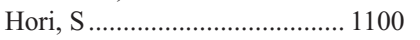 & Hui, A................... \\
\hline Hori, Т............... 1689, 1697, 1729 & ..........389, 724 , \\
\hline 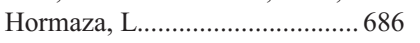 & $930,931,1024$ \\
\hline Horn, H ….............................. 1205 & ........................ \\
\hline Horn, $\mathrm{P} \ldots \ldots$. & Hukku, S ..... \\
\hline Horn, PS ................................. 1507 & 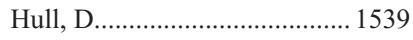 \\
\hline Horna, P .......................................... & 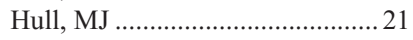 \\
\hline 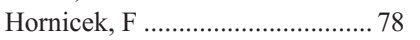 & Hulsbergen-van de Kaa, CA...... 906 \\
\hline$\ldots .38,50,375$, & Humphrey, P.................................. 904 \\
\hline $566,622,668$ & Humphrey, PA ......... 725, 726, 740 \\
\hline$\ldots \ldots \ldots \ldots \ldots \ldots . . .458$ & Humsi, MK ............................. 1239 \\
\hline 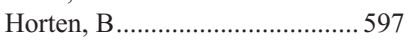 & Hungermann, D........................... 254 \\
\hline Horton, DL .................................. 1334 & Hunt, JL......621, 1121, 1127, 1129, \\
\hline 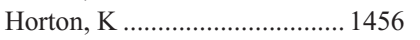 & $1143,1151,1152,1768$ \\
\hline Horvai, AE ....... 35, 41, 48, 51, 177 & Hunt, K.........................1196, 1218 \\
\hline Hosey, D........ & .....224, 231, 257 \\
\hline Hosone, $\mathrm{N}$ & 1500,1517 \\
\hline
\end{tabular}

Hunter, SV.

Huntsman, D

Huntsman, DG 1645

Huo, L

Hurd, DH

Hure, MC

Husain, AN .......... 1576, 1589, 1591, $1592,1602,1605$, $1616,1642,1644$

Husain, EA .................................. 459

Husain, S .................................... 463

Hussaini, M.................. 1132, 1140

Huston, A....................................... 311

Hutchens, KA.................... 460, 503

Hutcheson, IR …................... 162

Hutchinson, K ................................ 1750

Hutchinson, L.............................. 390

Huttner, AJ ................................ 1495

Hwang, E................................. 128

Hwang, HC .................... 290, 1296

Hwang, JE .......................... 993, 1000

Hy, J ........................................... 383

Hyland, J .................. 568, 581, 614

Iacobuzio-Donahue, CA........... 647 ,

1396

Iafrate, AJ ........... 1009, 1010, 1635

Iafrate, J........................................ 78

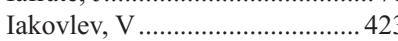

Ibanez, P...................................... 561

Ibarra, JA..................................... 201

Ibrahim, G....................... 202, 324

Ichihara, S ................................ 203

Ido, Y......................................... 1524

Idoate, MA ....................... 391, 1496

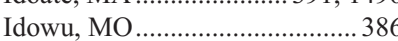

Idrees, MT ….................. 797, 828

Idress, M .................................... 883

Iezzoni, JC .................................. 1401

Iglesias, M.................................. 848

Iinuma, Y....................... 1606, 1697

Ijszenga, M................................. 85

IJszenga, M .................................. 92

Illei, P............................. 140, 1587

Illei, PB …................. 204, 490, 785

Imai, Y.................................... 1039

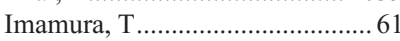

Inamdar, KV ............................... 1294

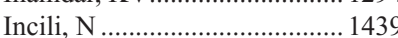

Ines, D .............................. 514, 515

Inghirami, G............................. 1711

Inomoto, C ............................... 536

Inoue, Y...................................... 630

International Non-Hodgkin

Lymphoma Classification

Project...

Inwards, CY .................................. 35

Ioffe, OB .......................... 272, 284

Ip, PPC .......................... 994, 1106

Iqbal, N ...................................... 319

Irahara, $N$.......... 578, 638, 642, 643

Irei, I...................................... 1286

Irish, JC ...................................... 1141

Irons, M.................................... 1495

Irons, R.................................. 1178

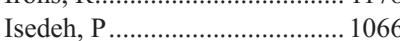

Ishida, M ............................. 1463

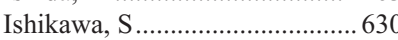

Ishikawa, Y................... 1623, 1624

Ishikura (deceased), H ............ 1005

Ishizawa, S ................... 1606
Ismiil, $\mathrm{N}$.................. 965, 986, 995, $1043,1046,1047$ $1058,1071,1072,1091$

Isola, J ............................. 181, 372

I-SPY Clinical Investigators ..... 235

Isse, K ...................................... 1454

Itani, D ........................................ 1338

Itani, DM................................... 42, 88

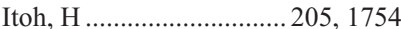

Itoh, T.............................. 205, 1754

Ittmann, M.................................. 727

Iuga, AC ....................................... 599

Ivanov, I ...................................... 947

Iversen, $\mathrm{K}$..................................... 461

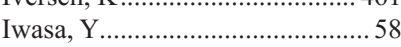

Iwata, Y .................................. 1606

Iwenofu, $\mathrm{H}$................................... 83

Iwenofu, OH ................ 1124, 1394

1395,1481

\section{$J$}

Jabbar, KJ ...................... 1219, 1220 Jackman, D.......... 1585, 1637, 1639 Jackson, J .................................. 1373 Jacobsen, JR ……...................... 1221 Jadallah, S ....................... 786, 803 Jaenisch, R .............................. 1535 Jaffar, R ........................................ 996 Jaffe, E .......................... 1195, 1303 Jaffe, ES …..................... 1239, 1291

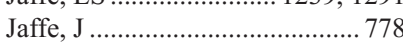
Jaffee, EM .................................. 1396 Jaffer, S ..................................... 206 Jain, A ........................................ 1026 Jain, D ................ 679, 1409, 1526 Jain, R ........................... 376, 520

Jain, S ....................................... 1376

Jakate, S ............................ 628, 649 Jakubowski, M............... 621, 1768 Jalil, YA.................................... 1212 Jamali, M....................................... 997 Jambhekar, NA.................. 82, 1603 Jan, S ........................................... 223 Jan, YJ ........................................ 983 Jang, JJ ...................................... 1420

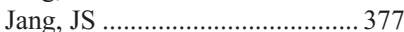

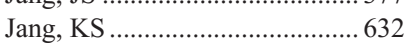

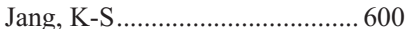
Jang, KT .......................... 659, 667 Jang, K-T........................................ 595

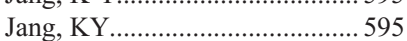

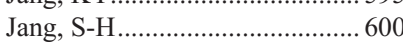

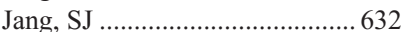
Jang, SM ......................................60, 632 Jani, JC ...................................... 787 Jani, P ........................................ 1172 Jannapureddy, SR …..........378, 998 Janne, P .............. 1585, 1637, 1639 Janne, PA................................... 1738 Jarboe, EA.............. 951, 971, 1076 Jarmulowicz, MK....................... 1539 Jarnagin, WR............................... 1389

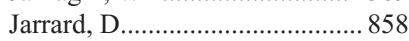
Jarzab, B................................... 528 Javed, R..................................... 788 Jaworski, J................................. 342

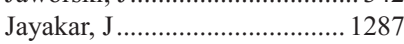
Jaye, DL ........................................ 1164 Jazwinski, M ............................. 1695

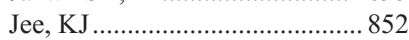
Jehnazeb, K ……….................... 787 Jeng, YM ............................................ 1032 Jenkins, CP............................... 1410 Jenkins, JR ................................ 1374 
Jenkins, SM.............................. 1489 Jennes, I........................................ 93

Jennings, TA....................... 808, 815

Jensen, CS …………………..... 1654

Jensen, KC …………………....... 289

Jeon, TY ………………………. 604

Jeong, JY ....................................... 699

Jevremovic, D ……………….... 1222

Jewett, M..................................... 905

Jhala, D ………………... 438, 1425

Jhala, N .......................... 618, 1425

Jhangri, GS...………………... 1705

Jhanwar, SC ………………….... 842

Jheon, SH ................................. 1613

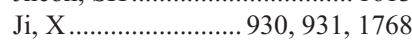

Jiang, A......................... 1172, 1223

Jiang, F ...................................... 1325

Jiang, W .......................... 999, 1014

Jiang, X ....................... 1224, 1253

Jiang, Z.......... 200, 996, 1494, 1542

Jiao, J........................................ 742

Jimenez, A ............................................ 120

Jimenez, R........................... 186

Jimenez, RE ...................... 697, 850

Jimeno, M .................................. 660

Jin, L..................................35, 633

Jin, T............................................ 1284

Jin, YT...................................... 1173

Jin, Y-T............................................. 683

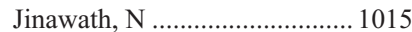

Jing, X .......................................... 146

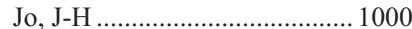

Jo, VY …….................. 728, 1146

Joensuu, H................................... 1547

Johansson, S ..................... 832, 980

John, R …………………….... 1375

Johnson, AT .................................. 868

Johnson, J ........................................ 1686

Johnson, JJ ............................. 1108

Johnson, M..................... 671, 1202

Johnson, MR ………………..... 1220

Johnson, MW ………………..... 1497

Johnson, SM............................... 1236

Johnykutty, S.................................. 207

Jokich, P.................................. 299

Jones, D...............1158, 1171, 1173,

$1259,1271,1289$

Jones, DM ....................... 565, 1191

Jones, G...................................... 827

Jones, JS …………..... 758, 879, 891

Jones, RL....................................... 44

Jones, S ............................................

Jones, T ................................... 1333

Jones, TD …………………..... 1649

Joo, M …………………....659, 667

Jorda, E ………………...... 475, 479

Jorda, M .................. 731, 779, 780

Jordan, CD ……………………....935

Jordan, M …………………….... 924

Jorgensen, JL................ 1191, 1192, $1220,1228,1246$

Joseph, AON ……..................... 1733

Joshi, V.................1585, 1637, 1639

Jouan, F .................................. 751

Jovanovic, I ................................... 383

Jover, R ……………………........546

Judson, G...................................... 760

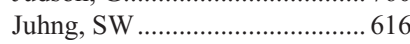

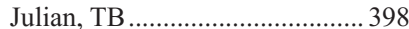

Jun, S-Y .......................................595

Jun, YJ....................................... 600

Jung, CK …………........ 1081, 1133

Jung, ES ................. 595, 1001, 1081

Jung, SJ ………………...... 595, 682

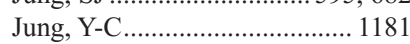

Jungbluth, AA ………....... 461, 481, $484,695,1552$

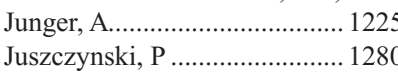

.. 1280

\section{K}

Kaag, M....................................... 892

Kabbani, W .................................. 1292

Kadin, ME.................................... 485

Kadow, B............................................ 379

Kageyama, S ....... 1606, 1689, 1729

Kahaleh, M.................................... 14

Kahana, D ……………………....529

Kahl, BS .................................... 1769

Kahn, AG ……………………......... 10

Kahn, ME .................................... 564

Kajdacsy-Balla, A ..................... 1716

Kajiura, D................................. 1320

Kajiwara, H............................... 1100

Kajiya, H......................... 536, 1734

Kakar, S.................... 624, 664, 665,

1411,1448

Kakiashvili, D ……………......905

Kalb, B ……………….....1398, 139

Kalimuthu, SN ............................ 22

Kallakury, BVS .................. 208, 789

Kalloger, SE ............934, 979, 1008

Kallwitz, ER.............................. 1405

Kalogeropoulou, C......... 1412, 1413

Kamatkar, S.............................. 1756

Kamel-Reid, S............................. 1247

Kaminetsky, J ..............................694

Kamionek, M .................................. 1542

Kamiyama, H ............................ 1427

Kamiyama, M ………………... 1427

Kanbour-Shakir, A....................... 209

Kandalaft, PL ..................290, 1296

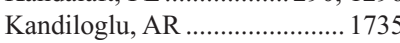

Kane, SV ................................... 1134

Kanellis, G ...............................1157, 1226

Kaneshiro, DK ..........................601

Kang, DY ……………….................. 54,602

Kang, GH ........... 54, 525, 595, 602 ,

$648,690,730,1420$

Kang, H................................. 1196

Kang, HJ .....................603, 604, 615

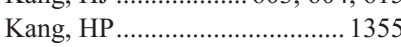

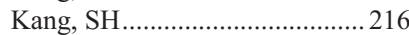

Kant, JA.................................................... 1199

Kantarjian, H............................. 1202

Kanthan, R .......................210, 504

Kantoff, PW …………………..... 885

Kao, YC ……............................ 1002

Kapadia, MJ .............................. 1414

Kapatou, K .............................. 1550

Kapels, KM ................................. 103

Kaplan, B …………………...... 1705

Kaposi-Novak, P ......................... 380

Kapur, P................................ 476

Kapur, U............................ 381, 433

Kapusta, LR …………………............... 827

Karabakhtsian, RG ....................2274

Karak, SG................ 202, 211, 324

Karamchandani, J..................... 1736

Karandikar, NJ ................ 1193, 1310

Karanikou, M ............................ 1230

Karanjawala, Z …………………1563

Karasu, Z .................................... 1439

Karatza, C ................................ 1468

Karberg, B .................................... 1077

Kardamakis, D ……......1412, 1413

Karim, N ................................ 1618

Karkouche, R ............................. 185

Karlan, B .................................... 1096
Karpate, AA.............................. 1134

Karperien, M ………………...... 1565

Karseladze, A .................................92

Karslioglu, Y ................................. 605

Kasai, K................................... 1482

Kasamatsu, E......................738, 739

Kasbidi, F .................... 1230, 1259

Kashima, TG ……………………...... 55

Kashima, Y............................... 1606

Kashyap, PC............................. 1040

Kasid, UN …………………....... 208

Kasper, KA.................................. 790

Kasprzycka, M ………………... 1189

Kassel, R ................................... 1401

Kasyan, A ...................................1326

Kasznica, J .................................. 331

Katabi, N ................................... 1391

Katayama, H ………………..... 1778

Katenkamp, D …………………...... 57

Katenkamp, K …………………....57

Katic, M .................................. 1047

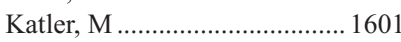

Kato, H..................................... 1135

Kato, N N.................................... 205, 1754

Katsuno, A................................901

Kattan, M .................................... 861

Kaul, D ......................................... 196

Kaul, KL.........................913, 1760

Kaur, P...................................... 1737

Kavuri, S ..................................... 382

Kawakami, F ................................... 1003

Kawasaki, T ................................. 641

Kayton, ML............................... 1571

Keating, MJ .............................. 1289

Keefe, MJ .................................... 484

Keegan, D ……………………..... 581

Keeney, GL ………….... 1041, 1086

Kehoe, JM ……………………...... 729

Keller, CE...………………...... 1485

Keller, G............................................ 1298

Keller, RA …….....................1330

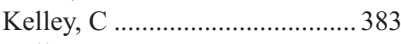

Kelley, MJ .................................... 88

Kelley, TW ………….................1221

Kelly, D ....................................... 1774

Kelly, MM ................................ 1608

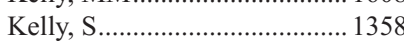

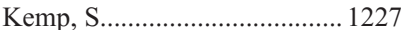

Kemper, C ........................................ 426

Kempson, RL ……………........ 1028

Kendall, BS ................................. 404

Kennedy, LA …………………….... 335

Kennedy, SM...............................212

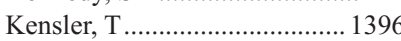

Kepler, CY ……………………. 549

Kerble, P........................................ 353

Kerfoot, BP …………………......505

Kerley, S..........................369, 393,

394, 427, 1061

Kern, SE .................................... 1396

Kernochan, LE ................................ 1414

Kerr, SE..................................... 14

Ketterling, RP...... 1086, 1162, 1217

Keung, Y ................................. 1161

Kevans, D.........................568, 581

Keylock, JB ................................ 791

Khalbuss, W ..............396, 399, 750

Khalbuss, WE............357, 397, 1356

Khalbuss, WK ………………...... 356

Khalifa, MA .............................356, 986,995 ,

1043, 1046, 1047, $1058,1071,1072,1091$

Khan, A ………………..... 189, 237

Khan, FM …………………......... 1682

Khan, SA ............................ 172, 308

Khanna, S ................................... 484

Khayyata, S ....................... 672, 792

Khelifa, S ............................... 213

Khilko, N...................................214

Khiyami, A .................................. 407

Khokhar, FA................... 1228, 1259

Khoury, N.................................. 494

Khoury, T ...................113, 164, 215,

$675,676,1609,1759$

Kida, M .................................... 1653

Kiefer, S ................................. 1295

Kiehl, T-R................................. 1498

Kiemeney, L …………………...... 906

Kieran, M ................................. 1495

Kilgore, M.................................. 1765

Kilic, M ................................. 1439

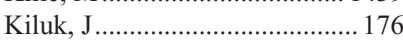

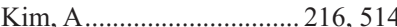

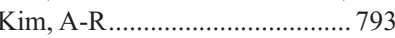

Kim, AW .........................199, 1341

Kim, B .........................1499, 1509

Kim, BH.............................. 1420

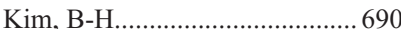

Kim, DH............................ 604, 604

Kim, DJ _................................ 1603

Kim, DS ............................................ 586

Kim, DSL .................................... 468

Kim, DT …………………….... 1229

Kim, DY ……………………............ 616

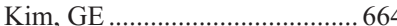

Kim, GH................... 603, 604, 615

Kim, GI .................................... 595

Kim, H.................................. 840

Kim, HJ .................. 875, 919, 1690

Kim, H-S................................. 56

Kim, HY …………….......604, 606

Kim, J.......... 157, 1375, 1546, 1610

Kim, JE ........................ 1217, 1230

Kim, JG ......................................... 606

Kim, JH .................... 595, 606, 730

Kim, JM ……............................. 595

Kim, J-M ………………............ 793

Kim, JS.............................606, 617

Kim, JY ...................217, 218, 595

Kim, KE ................................. 852

Kim, KM ………….... 54, 602, 607,

$608,659,667$

Kim, KR ................682, 1051, 1085

Kim, K-R.............. 993, 1000, 1020

Kim, MJ ……………………......... 794

Kim, M-J ……………………..... 793

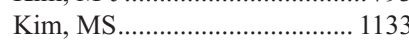

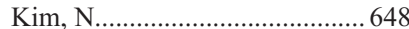

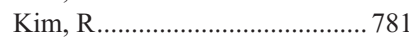

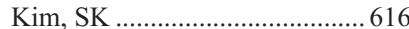

Kim, SS ................................... 1917,795

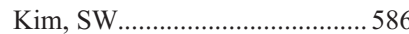

Kim, WH......................................... 690

Kim, Y ………………………...... 617

Kim, YD ...................................... 303

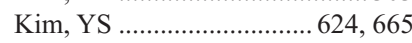

Kim, YW …………………....... 102

Kimler, BF ................................ 673

Kindblom, L-G.............................. 90

Kindelberger, D.........................1111 
Kishi, K ...................................... 1599 Kiss, L ...............................429, 846

Kiss, LP..................................... 928

Kiss, S .....................................60 609

Kitabayashi, N............................416

Kitahara, S.................... 1004, 1232

Kitayama, H ............................... 836

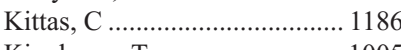

Kiyokawa, T............................. 1005

Kjeldahl, K....................... 370, 413

Klassen-Fischer, MK ............... 1336

Klaustermeier, J ...... 738, 739, 1026

Klco, JM................................. 1233

Kleer, CG .......... 147, 225, 226, 325

Klein, AP .................................. 1458

Klein, E ………………757,925

Klein, ME................................... 1006

Klein, MJ....................................... 36

Kleiner, DE .............................. 1430

Kleinschmidt-DeMasters, BK........

Klement, P................................. 962

Klimberg, S …………………..... 158

Klimowicz, A ..................1253, 1691

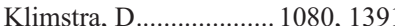

Klimstra, DS …………....677, 1389,

1390,1461

Klimtchuk, E............................. 1200

Kloppel, G....................................533

Kluk, M .................................. 1234

Kluk, MJ …………………….... 1235

Knesek, M ................................. 770

Knezetic, J................................. 1531

Knisely, AS .............................. 1415

Knosel, Th.....................................57

Knowles, DM...... 1170, 1180, 1324

Knudson, RA............................ 1162

Ko, DS....................................... 1363

Ko, V ................................. 384

Kobel, M .......934, 979, 1007, 1008

Koch, I................................... 1298

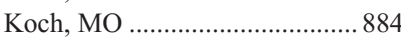

Kodjoe, E ………………………. 758

Koenig, C .................................. 134

Koeppen, H ..................... 446, 1326

Koepsell, SA ………………….....15

Kogos, A …………………….................. 1702

Kohr, J ...................................... 106

Kojima, A …………………….... 1003

Kojima, M ................................ 1328

Kojiro, S....................... 1009, 1010

Kolar, GR …………………….... 1527

Kolch, W ................................. 572

Kologinczak, T............................. 381

Komminoth, P ……………….... 533

Kondratiev, S................... 385, 1236

Kong, CS .................................. 548

Kong, K....................................... 327

Konishi, E ……………………..... 58

Konishi, Y .................................. 775

Konstantinidis, I............................... 1416

Konvalinka, A ………………....1375

Kooby, D ......................... 672, 1399,

$1443,1444,1465$

Koplin, SA...................................... 59

Korbel, JO …………………..... 1677

Korcheva, VB................................. 16

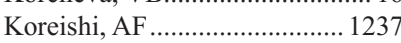

Korner, G …………….......... 1209

Korner, M.................................... 610

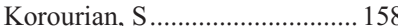

Koru-Sengul, T........................... 1104

Kosemehmetoglu, K ...................... 60

Kossover, CL............................. 1238

Kostopoulou, E ......................... 1011
Kotnis, G ................................... 1166 Kou, ZC.................................. 1579

Koukoulis, G ................................. 1011

Koukoulis, GK ………………... 1550

Kouprina, N............................... 1554

Kovach, AE ............................... 1239

Kowalewska, J .......................... 1379

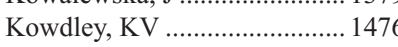

Kozakewich, HP...…………….... 353

Kozono, DE......................... 76, 247

Kraemer, M ………………….... 438

Kraft, P ........................................ 642

Krag, DN .................................... 230

Krane, JF .........................375, 1111

Krasik, EF ................................. 1012

Krasinskas, A.................. 672, 1465

Krasinskas, AM …………..... 592, 611

Krastins, B................................... 313

Kraus, JA.......................1013, 1078

Kraus, RK …………..... 1500, 1501

Kraus, TS .......................220, 1501

Krause, RS .................................. 478

Krausz, C.................................... 842

Krausz, T....145, 1576, 1589, 1591, $1592,1602,1616,1642$

Kreike, B …………………….... 322

Kreisel, F.....................1233, 1261

Kremer, M..................... 1298, 1313

Kretzler, M ................................ 1372

Kreuter, JD …………………........ 612

Krill-Burger, JM............................... 928

Kris, M ....................... 1595, 1596

Krishnamurthy, S ......221, 222, 224

Krishnamurti, U ..............223, 1770

Krishnan, B …………………..... 788

Krishnan, JA.................. 1592, 1602

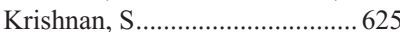

Kristiansen, G ............................... 853

Kroft, SH.................... 1206, 1309 ,

1728,1746

Kroll, T................................... 1122

Kroon, HM.............................97, 98

Krop, IE................................... 1738

Krous, HF ........................................ 3

Krouse, R ........................................ 495

Krupinski, EA …………………...507

Kshirsagar, M...............................999

Kshirsagar, MP......................... 1014

Ktori, E......................................... 715

Kuan, SF ……………………....591

Kubes, P ……………………. 1608

Kudahetti, S.................................. 715

Kudo, E ..........................538, 657

Kuefer, R ......... 781, 853, 854, 1677

Kuhn, E ………………..... 1540

Kuick, R …………….... 1668, 1684

Kulesza, P..................................302

Kulkarni, A................................. 1726

Kulkarni, RS ................................796

Kulkarni, S ................................2215

Kulkartni, S .................................. 164

Kumagai, A ……………………..... 61

Kumar, AM ………………………. 5

Kumar, S ………………….... 438

Kundu, A ................................... 1771

Kundu, UR ………………….....224

Kunju, LP .......... 225, 226, 873, 911

Kuntzman, TJ ........................... 1558

Kuo, F …........................ 55

Kuo, KT ................. 999, 1015, 1016

Kuo, K-T ................................. 1525

Kuo, L-T................................. 1525

Kure, S .............. 578, 638, 642, 643

Kurman, R..................................... 1105
Kurman, RJ .......... 999, 1014, 1015, $1016,1060,1078,1101$

Kuroda, M ............................... 1149

Kurosawa, S ........ 1331, 1339, 1348

Kurosumi, M ...............................260

Kurtin, PJ ..................... 1184, 1222 ,

1277,1307

Kussick, SJ ..................... 290, 1296

Kusuanco, D............................. 1604

Kutok, JL.......................1280, 1319

Kutys, R ………………….... 8, 336

Kutzner, H.......................... 457, 473

Kuwada, SK ………………….... 562

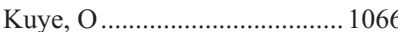

Kvols, L.................... 518, 519, 1708

Kvols, LK................................ 1406

Kwon, KW …………………….... 595

Kyshtoobayava, A .....................1758

Kyshtoobayeva, A ....................... 126

\section{$\mathbf{L}$}

La Quaglia, MP......

1571

1417

Labrousse, FJ ................................... 1490

Lacroix-Triki, M ..... 125, 227, 1692

Ladanyi, M....................1128, 1563

1571,1633

Ladd, AC .................................... 1693

Ladenheim, HS ............................ 386

Ladner, J...................................1495

Lafargue, C.................... 1674, 1677

LaFargue, CJ ………….....743,853

LaFargue, CL .............................. 1648

Laforga, J ................ 110, 228, 261

LaFramboise, WA...............517, 928

Lafyatis, R................................1377

Lagoo, AS …………………....... 1207

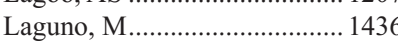

Lahat, G..................................... 81

Lai, KK ……………………......... 613

Laios, A ……………….....970, 976

Laird, PW ……………………....2292

Lakey, MA................................ 1240

Lal, A......................................... 128

Lalor, RJ ……………………...... 1727

Lam, M.................................. 1418

Lamb, CA.........................1017, 1018

Lamb, J J............................. 1699

Lambe, JS.................................. 1477

Lambros, MBK ......... 175, 240, 254

$255,277,1506$

Lamps, LW ........................619, 655

Lance, MP ............................... 549

Landolfi, S.................................... 1557

Lang, TU ……………………. 1611

Lange, PH ..................................... 899

Lange, RA …………………....... 797

Lankester, AC.................................. 37

Lannert, KW................................. 443

Lannes, B ……………………..... 281

Lanotte, M.................................. 1508

Lantuejoul, S............................ 1612

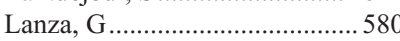

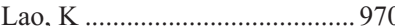

Lapetino, S .................................. 851

Lapinski, J .................................. 798

Laporte, K ................................ 1200

Lareau, M................................. 1224

Laronga, C.........................176, 243

Larson, AM …………………..... 1462

Larson, RA ………………....... 1208

Lasho, TL .................................. 1240

Laskin, W ...................................... 43

Laskin, WB ……………………... 443

Lasota, J . $62,63,72$

Lassige, K .............................. 1358 Lassman, CR ....... 1431, 1438, 1447

Lathrop, S..................................... 20

Latorraca, EF............................. 1510

Latour, M .......... 698, 799, 800, 813

Lau, C..........................1571, 1633

Lau, S ………………………..... 378

Lau, SK ............................... 1177

Laucirica, R..................... 359, 1119

Laude, H................................... 488

Lauer, S ………………....229, 462

Laurent-Puig, P ........................ 1740

Laury, AR …………………..... 1241

Lauwers, G........................................... 598

Lauwers, GY ............ 555, 556, 603, $604,639,640,653$, $669,1416,1429,1452$

Laver, NM ............... 111, 385, 1522

Law, K................. 1553, 1583, 1635

Law, ME.......................1242, 1308

Lawler, J...........................528, 1559

Lawrence, HJ ............................283

Lawrence, WD ................ 392, 1048 ,

$1054,1061,1082$

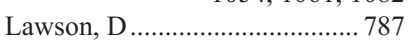

Laycock, N................................... 836

Lazar, AJ ........................... 44, 457,

$711,712,721$

Lazar, AJF ....................... 64, 80, 81

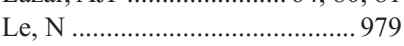

Le, UP ……................................. 230

Le Corre, D ................................. 1740

Le Stang, N ………………….... 1600

Leahy, DT...................................... 614

Lear, S ……………………....... 486

Leary, RJ …………………...... 1396

Leathersich, AM................................. 801

Lebanony, D ....................1586, 1701

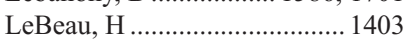

Lechowicz, MJ ....................... 1290

Lechpammer, M ........................... 871

Lecksell, K .................................. 338

Lee, A ............................1001, 1081

Lee, AHS ..................................... 239

Lee, C................... 1585, 1639, 1677

Lee, CH .......... 603, 615, 1019, 1669

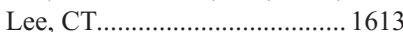

Lee, EB ………………………..... 1502

Lee, H........................................ 1419

Lee, HJ .......................... 1613, 1650

Lee, HS ............................690, 1420

Lee, J ..................................... 1765

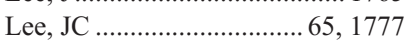

Lee, J-E ................................ 1421

Lee, JH ...... 219, 603, 615, 616, 617

Lee, JJ .................................... 1020

Lee, JR .........................................54

Lee, JS.......................219, 595, 616

Lee, KE .................................... 1636

Lee, KH...............600, 616, 617, 632

Lee, KL .................................... 463

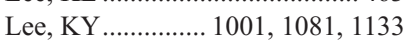

Lee, P …………..... 579, 766, 783,

$845,921,1407$ 
Leffler, D .................................... 635 Lefkowitch, JH............... 1470, 1477 LeGallo, RD .................................. 14 LeGolvan, MP........................... 1243

Lehman, CD ………………….... 106

Lehrkamp, M............................... 260

Lei, T................................................ 571

Leiman, G …………………...... 366

Leite, KRM ………………….... 1694

Leith, CP ………………......... 1769

Leizerman, I ...................1586, 1701

Lekakis, L................................ 1158

Lekili, M ................................... 1735

Lele, SM.................. 832, 980, 1059

Lemarbre, GB ……………...... 1262

Leng, X …………………….... 1533

Lennerz, JKM ............... 1376, 1422

Lennon, P ................................ 1219

Lentz, MR …………………….... 196

Lenz, G...................................... 1258

Leon, M.................................... 1124

Leong, WL ................................ 423

Leopizzi, M ................................ 747

Lerma, E..................................... 270

Lerner, SP........................................ 874

Lerwill, MF .............................. 1036

Lesieur, B ...................................... 109

Lessi, F...................................... 747

Lester, TR......................... 231, 257

Leszl, A ……………………....... 1562

Lett, GS ..................................... 1762

Leung, CS …………………….... 827

Leung, S .............1386, 1480, 1645

Leung, YK.................................... 766

Lev, D.................................. 64, 81

Levanon, K..................... 1021, 1022

Levenson, R .............................. 1538

Leventaki, V ............................. 1158

Levi, E.................... 494, 672, 1465

Levi, G ...................................... 599

Levin, M....................... 1156, 1244

Levin, MB ................................ 1293

Levy, G........................................ 464

Levy, GA..................................... 1387

Levy, M................ 564, 1423, 1424

Levy, R ..........................1249, 1257

Levy, S ...................................... 1249

Lewin, DN................................... 361

Lewis, J ………………………......... 1480

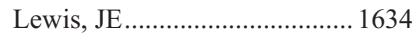

Lewis, JS ................................... 1140

Lewis, MR................................. 1250

Lewis, MT …………………….... 232

Lewis, VO ..................................... 99

Lewis ,Jr, J ................................ 1132

Lewis, Jr, JS ............................... 1120

Leyva, WH................................... 478

$\mathrm{Li}, \mathrm{A}$............................................ 1547

$\mathrm{Li}, \mathrm{CF}$............................ 53, 65, 66

Li, C-F ......................................... 683

Li, C-S................................... 276

$\mathrm{Li}, \mathrm{DQ}$........................................ 1651

Li, F................................. 1598

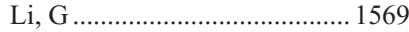

Li, HX .................................. 238

Li, J …..............511, 513, 521

Li, L.................. 8, 336, 1614, 1615

$\mathrm{Li}, \mathrm{M}$............... 465, 514, 805, 1616

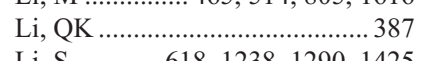

Li, S............618, 1238, 1290, 1425

Li, W ..... 577, 675, 676, 1384, 1759

Li, X .......................232, 303, 567

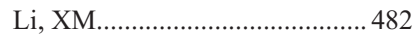

Li, Y .........................233, 313, 522, $783,1165,1492,1570$

Li, YR............................766, 921

Li, YX .......................................... 626

Liakou, C................................. 1158

Lian, B...…………………..... 466

Lian, C......................................466

Lian, E.................................................... 334

Liang, $\mathrm{H}$..................................... 570

Liang, J.................................... 1426

Liang, M......................... 806, 1245

Liang, Q ....................................... 67

Liang, W............................................ 817

Liang, $X$ …………………......... 1166

Liao, J.........558, 627, 637, 650, 651

Liao, S ..................................... 174

Liao, S-L ......................................... 1525

Liapis, H.................................. 1376

Liava, A ......................................... 691

Lifschitz-Mercer, B ................... 1089

Ligon, A................................. 1683

Ligon, $\mathrm{AH}$................................. 1738

Lilla Della Monica, P.................. 346

Lim, HJ .................................... 1455

Lim, J …………………….... 417

Lim, KH ................................. 1464

Lim, M ...................... 468, 469, 807

Lim, MS ............. 1179, 1252, 1681

Lim, P......................... 1427, 1457

Lim, S-J..................................... 56

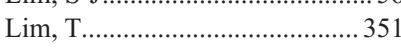

Limeres-Gonzalez, MA............. 1282

Lin, C-H .................................. 1525

Lin, CY..................................... 784

Lin, DW ………………......... 899

Lin, E...………… $388,420,870$

$1025,1424,1533$

Lin, $\mathrm{H}$......................................... 1533

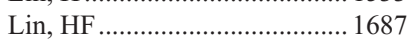

Lin, JC ..................................... 1396

Lin, MC ............... 1002, 1023, 1033

Lin, M-C ……………………..... 1525

Lin, MT ……………………..... 1427

Lin, O ...................................... 371

Lin, P...................549, 1203, 1204,

$1219,1246,1276,1494$

Lin, $X \ldots \ldots \ldots \ldots \ldots \ldots \ldots \ldots \ldots . . . . . . . . . . . . . . .43,1561$

Lin, Y ………………………........ 195

Lin, YC....................................... 1534

Lin, Y-T .....................................1525

Lindberg, MR ................... 619, 1136

Lindeman, N ................. 551, 1585 ,

1635,1639

Lindeman, NI ...........................1637

Linden, MD ..................................... 68

Lindgren, BR..............................547

Lindholm, PF ............................... 817

Linehan, WM ..................... 809, 866,

Linford, R................................... 155

Ling, SZ ……………………....... 803

Linos, K................................. 815

Linos, KD............................... 808

Lipata, F ……………..... 389, 1024

Liptay, M.................................. 1341

Liptay, MJ ……………………..... 199

Lirola, JL .................................. 269

Lisanti, M..................................... 325

Lisker-Melman, M ................... 1233

Lisovsky, M...................640,1429

Lissowska, J ................................ 292

Little, D …………………….........235

Liu, A ……………………….... 1778

Liu, AJ...................................... 1541
Liu, C ……………………….... 1150

Liu, F ........................................ 331

Liu, F-F .................................. 1135

Liu, GZ .....................................950

Liu, H ..................... 234, 388, 420

1025,1150

Liu, J .................... 963, 1050, 1107

Liu, JJ..................................... 620

Liu, JS ……........................... 950

Liu, L............................ 383, 1548

Liu, M …………………........ 114

Liu, P ...................................... 1107

Liu, Q ……............ 809, 1428, 1494

Liu, W ……………....317, 930, 931

Liu, X ...................... 621, 1189, 1198

Liu, XM................................... 921

Liu, Y …………………............... 898,828

Liu, YL .................. 432, 1531, 1726

Liu, YS ...................................... 238

Liu, YX ……………………… 390

Liu, Y-Y....................................... 1695

Liu, Z ………………................... 1201

Liu Yin, JA .................................1707

Livasy, CA.........................130, 235

LiVolsi, VA.................... 1045, 1126

Livolsi, VA ..................... 1137, 1138

LiVolsi, VA................................ 1147

Lleonart, M ............................... 1688

Llobet, D ……………………..... 1042

Llorente-Cortes, V ..................... 948

Lloveras, B......738, 739, 974, 1026

Lloyd, RV ....................35, 450, 540

Lockheart, K .............................. 674

Lockwood, G...................... 755, 904

Loda, M................. 760, 902, 1553, $1651,1670,1683,1699$

Loda, MF.................................. 1738

Lodhi, A......................................... 221

Loevner, LA .............................. 1138

Loftus, B ............................................ 714

Loftus, M.........................260, 1762

Logan, N ................................... 1432

Loganathan, S ........................... 1589

Logani, S ........................ 998, 1359

Loghavi, S ............................... 1027

Lohse, C ...................................... 540

Lombardi, L ............................... 1026

Lones, MA................................. 1268

Long, KB..................................... 622

Longacre, TA...................... 548, 957,

$958,1028,1074$

Longatto-Filho, A..................... 1506

Longtine, J................................ 1495

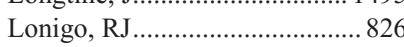

Lonigro, RJ …………………..... 824

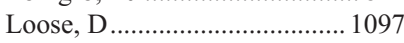

Lopategui, J.............................1083

Lopes, JM................................... 1506

Lopez, D................................... 1647

Lopez, JI........................... 810, 811

Lopez, MF ................................... 313

Lopez, S ................................... 1075

Lopez-Beltran, A..... 729, 812, 1649

Lopez-Guerrero, JA..................... 475

Lopez-Guillermo, A ...... 1167, 1265

Lopez-Ruiz, P.............................. 810

Lopez-Terrada, D .............. 64, 1486

Lopez-Terrada, DH ........................ 80

Lord, CJ...........................244, 255

Lorsbach, R .............................. 1169

Lorsbach, RB _.................. 1168

Losada, H ....................................1392

Loseph, L …………………......... 1555

Loss, GE..................................... 1330

Lossos, IS ...................... 1249, 1257

Lotan, T...................................... 698

Lotan, TL........................... 813, 814

Lou, J......................................1247

Loughman, NT ..............................400

Louissaint, A ……………….... 1351

Lovitch, SB ………………….... 523

Low, P .................................. 1080

Lowe, AC ............................................. 236

Lowery, FJ............................. 1717

Lowery-Nordberg, M ..................1696

Lowichik, A.............................. 1566

Loya, T ........................................ 362

Loyd, EJ ………………………......... 623

Lozano, JJ …………………….... 167

Lozano, MD ........... 391, 1496, 1626

Lu, D ............. 200, 996, 1494, 1542

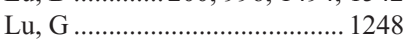

Lu, S................. 237, 467, 808, 815

Lu, T ....................................... 328

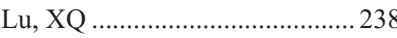

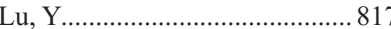

Luan, C......................................... 790

Lucas, D ……………………..... 1617

Lucas, PC ................................... 1681

Lucchesi, C ……………………... 185

Lucci, A.............................221, 222

Luce, J .................................... 1698

Lucia, MS.................................506

Lucia, VC .................................... 294

Lueck, NE ……………………..... 816

Luff, RD ..................................... 426

Lui, PC ……………………..... 1503

Luna, MA ......................... 539, 1117

Luna, S ..................................... 120

Lunardi, F................................. 1588

Lunde, $\mathrm{JH}$................................. 1250

Lundquist, KF ............................ 470

Lung, D ............................................. 732

Luo, G ........................... 544, 1618

Luo, H-Y .................................. 1236

Luo, RF …......................... 1249

Lupski, JR ................................. 1486

Luster, JS.................................. 1721

Luthra, MG ................................. 626

Luthra, R .... 626, 1171, 1203, 1230 , $1259,1289,1492,1773$

Luthringer, DJ ........................... 1543

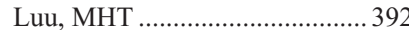

Luzzatto, L …………………...... 842

Ly, A ................................384, 1028

Lykes, W …………………….... 940

Lyle, M...................................... 1542

Lyman, G...................................... 311

Lynch, HT ……………………........ 563

Lyon, E...…………………......502

Lyons-Weiler, MA............................. 517

Lytwyn, A.......................... 161, 1093

\section{M}

Ma, C........................... 1062, 1449

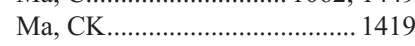

$\mathrm{Ma}, \mathrm{CL}$......................................... 238

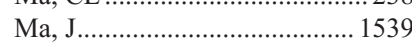

Ma, L..... 468, 469, 472, 1315, 1316

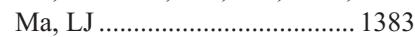

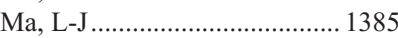

Mac Grogan, G.......................... 125

Macarenco, RS ........................... 69,450

MacGrogan, G............................ 281 


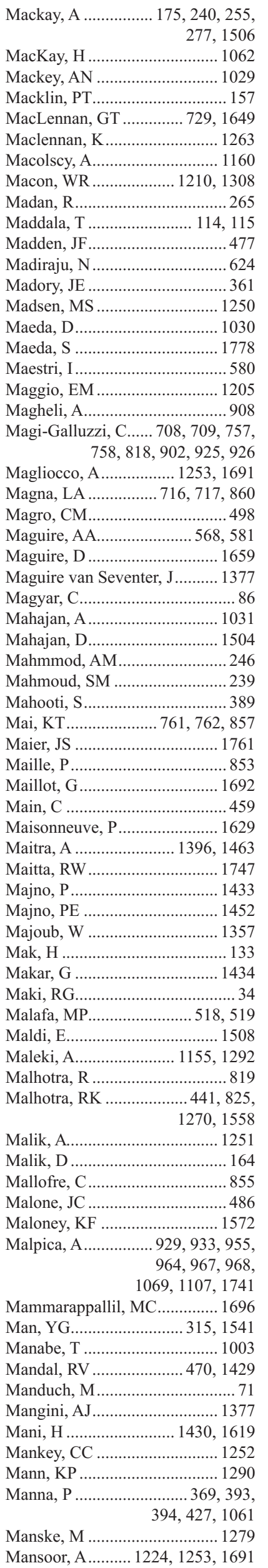

Mansukhani, M ........................... 678 Mansukhani, MM........................ 463 Mantha, GS ………………….......592 Mantilla, JC................................. 17 Mantilla-Hernandez, JC ............... 18 Manuyakorn, A............... 1431, 1432 Manzo, A …………………........ 327 Manzotti, M...................................1629

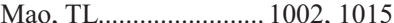
$1016,1032,1033$

Marais, R................................... 447 Marchet, S....................1306, 1417 Marchetti, AL ........................... 1719 Marchevsky, AM.... 395, 425, 1603 , $1604,1620,1621$

Marchio, C ……..240,254,255 Marckmann, P.............................. 25 Marginean, EC …………... 674, 761,

762,857

Mariani, A ………………….....104

Mariani, L.................................. 1026

Maric, I..................................... 1254

Marin, M ............167

Marinescu, M ..............................241

Marino-Enriquez, A................... 1034

Mark, EJ …………………….... 1623

Markey, J..................................235

Marks, RS ………………….... 1634

Marks-Jones, D ..................396, 397

Marrades, R............................. 1632

Marsee, DK …………………..... 1255

Marsh, WL _......................... 689

Martel, M .................................. 1024

Marti, RM …………………...... 492

Martignoni, G.................... 722, 723,

$820,821,840$

Martin, A ……………………...... 486

Martin, C..................................... 970,976

Martin, CM ………….......358, 937 ,

1549,1715

Martin, D................1398, 1399

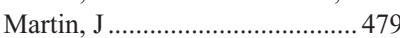

Martin, K..................................... 980

Martin, MA ………………….... 1656

Martin, MG …………………….... 529

Martin, SG . $\ldots \ldots \ldots \ldots \ldots \ldots \ldots \ldots \ldots . .246$

Martinez, A....................1160, 1195, $1265,1332,1345$

Martinez, AA............................... 704

Martinez, D ………………..... 2, 340

Martinez, EA................................ 269

Martinez, JC ................... 1505, 1544

Martinez, N ………………..... 1262

Martinez, OV …………………... 1745

Martinez-Penuela, A.................1626

Martinez-Penuela, JM ............... 1626

Martinho, O...............................1506

Martini, S .................................. 1372

Martins, A................................... 1506

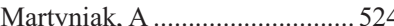

Maru, DM ………...... 584, 625, 626

Marulli, G............................. 1588

Maruno, T................................... 1697

Marwaha, J ..................................... 63

Marwaha, JS................................... 46

Marzec, M ...................... 1189, 1198

Masako, M ................................242

Masieri, L ................................................. 747

Mason, L …………………......... 694

Massey, CC ……………………....... 361

Mast, K ………

Mastrodomenico, P .................. 1391

Mathers, J................................ 1547

Mathew, R ...................................243

Matias-Guiu, X ..... 492, 1042, 1075
Matoso, A ........................915, 1622 Matsubara, O............................ 1623

Matsumura, T ……………………... 74

Matsuyama, A ………………….... 49

Matteuzzi, M................................. 580

Mauer, M................................... 1378

Maurer, MJ ............................... 1163

Maxwell, L............................... 1608

Mayer, I .............................296

Mayo, MM ………………………... 32

Mayordomo-Aranda, E ................. 64

Mayorga, M.................................. 492

Mazurek, A................................1602

Mazzoleni, G........................ 661

McAlhany, SJ ................. 1035, 1036

McAllister-Lucas, LM ..............168

McCardle, TW ............................. 471

McCarthy, A ................................244

McCarthy, S .............................. 1406

McCarthy, SM .............................250

McCarthy, SW............................470

McClelland, RA ............................ 162

McClintock, DS …………........ 1742

McClure, RF ............................. 1163

McConnell, TG …................. 1037

McCready, DR ………..... 423, 1556

McCue, PM …………………... 1475

McDavit, J....................................79

McDermott, S........................... 1707

McDiarmid, SV ........................1431

McDonald, AG ................ 1038, 1039

McElligott, HS _..._.................627

McEvoy, L.................................... 937

McGrath, M........................... 1170

McGrath, P ................................ 136

McGrath, PC ...............................2274

McGregor, DH ............................ 673

McGuire, A................................. 1256

McHugh, JB _...1127

McInerney, J..................................358

McIntire, M _................628,649

McKenna, EF …………………....283

Mckenna Jr, RJ........................ 1604

Mckenney, JK ……………….......712 712

McKenney, JK................... 735, 736,

$822,823,850$ $856,859,1028,1074$

McKenzie, PR ……………..... 400

McLeod, DG …………………….... 764

McLoed, DG …………………...... 765

McMahon, L................................182

McMahon, LA....... 472, 1471, 1472 ,

McManus, B...………………..... 35

McMeekin, DS ………........ 1108

McMillen, BD ............................ 1555

McNiff, J ...................................464

McPhaul, LW ...……………..... 1099

Meadows, JT .................................. 12

Means, J ......................................296

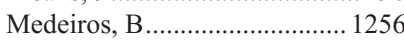

Medeiros, F ……….... 1040, 1041

1056,1070

Medeiros, J ......................... 1326

Medeiros, LJ ........... 153, 446, 1158 $1185,1186,1191,1192$ $1202,1203,1204,1217$ $1219,1220,1228,1230$ $1245,1259,1260$ $1276,1289,1294$

Medina, AM ................................. 361

Medlicott, SAC ………………...... 589

Meehan, SM .......................24, 1381

Meeker, A ……………………..... 895

Meeker, AK..... 772, 775, 813, 1554
Meeuwissen, JC ……………….... 554 Mehl, E................................ 1455

Mehra, R .......... 773, 774, 824, 853

Mehrotra, S ……………..... 374, 433

Mehta, A .................................... 1618

Mehta, JP....................................212

Mei, Y.............................. 1703

Meier, FA.................................... 1657

Meijer, CJ _............................. 1646

Meining, A..................................... 590

Meir, A ……………………… 585

Meirelles, L.............. 716, 717, 860

Meiri, E ……………………... 1630

Meitner, P ………………..... 871

Mejias, A ....................................... 279

Melachrinou, M......................... 1297

Melamed, J................ 766, 783, 845,

$921,1655,1702$

Melan, MA ................................. 1199

Mellado, B...………………….... 855

Melnyk, N ………………….......256

Melora, PR …………………......1571

Melo-Uribe, MA ………………..... 18

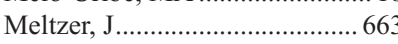

Members of the Pathology

Committee of the ERSPC ...904

Mena, F ...................................56

Mendez, E ……………………... 362

Mendiola, M................................. 1034

Menendez, C ..........................1026

Menestrina, F .......... 722, 723, 1711

Mengshol, SC ............................ 197

Menicagli, M.................................. 747

Menon, A..................................... 826

Menon, SP .................................. 1163

Mensink, KA................... 483, 1070

Mentha, G .................... 1433, 1452

Mentrikoski, MJ ............................ 472

Mentzel, T …………….....457, 473

Merati, K ................................... 1170

Mercado-uribe, I …................ 1107

Merchant, M............................. 1571

Merchant, S ................................ 703

Merchant, SH ....................704, 825

Mercurio, A ……………………... 237

Meredith, D ……………….........20

Merha, R ……………………..... 826

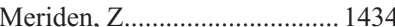

Merino, MJ................. 105, 809, 866 $907,1052,1709$

Merker, J ................................1315

Merker, JD …………………….... 1671

Merrimen, JL_...…………....... 827

Mertens, BJ ……………………......... 97

Mertens, F …u-

Mery, CM ……………………...... 247

Mesa-Tejada, R .........................1682

Meseguer, A .................................. 893

Meshberger, CL...…………….... 729

Messerini, L …………….............661

Messina, JL ……………………............ 471

Mestre, MJ ............................. 1283

Metcalf, RA............................... 1257

Methorst, N ………………….... 554

Meyer, JS ………………………. 245

Meyer, PN ..................... 1201, 1258

Meyer, R..........................663, 1756

Meyer zum Bueschenfelde, C.........

Meyerson, HJ ............................ 121

Meyerson, M …_......1648, 1677

Mhawech-Fauceglia, P................ 701

Mian, B ...................................... 815

Mian, BM ……………………..... 808

Middleton, LP ............................ 169 
Mierau, G

Miettinen, M .

1776

..... 62, 67,

Mihm, MC................................ 476

Mihm, MJ................................. 470

Mikami, Y ................................ 1003

Mikulasovich, M....................... 914

Mikulasovich, MP..................... 828

Mikuz, G .................................. 812

Milanezi, F .............................. 1506

Milea, A..................................... 1704

Miles, L....................... 1423, 1507

Miles, MV ............................... 1507

Milewski, M............................ 614

Milito, C....................... 1230, 1259

Miller, C ................................... 1686

Miller, DV .............27, 30, 31, 350

Miller, EC............................ 73, 474

Miller, J .................................... 685

Miller, JS ......................... 804, 829,

$830,831,834$

Miller, JW ................................. 276

Miller, K....................................... 280

Miller, L ..................................... 883

Miller, LJ.................................................. 610

Miller, N..................................... 423

Miller, P.................................... 1762

Miller, R ....................... 832, 1059

Millington, KA......................... 1344

Mills, CJ................................... 629

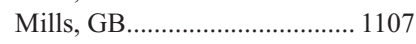

Mills, SE ......................... 170, 1146

Milnes, JT................................ 1716

Min, HS ...................................... 525

Min, K-W .................................. 600

Minervini, A................................ 747

Minervini, R.............................. 747

Minett, WJ................................... 21

Mino-Kenudson, M..........639, 640, $1360,1429,1435,1441$

Minot, DM …........................ 1743

Miquel, R ................................. 1436

Miranda, RN ................. 153, 1260

Mirani, NM .............................. 1521

Mirocha, J ..................... 514, 1604

Miron, A....................... 1056, 1067

Misdraji, J ............................... 1423

Mishellany, F............................. 125

Mitani, K ................................... 1404

Mitchell, S.................................. 155

Mitsuhashi, T ............................. 74

Miura, T .................................... 1482

Miyamato, H .............................. 749

Miyamoto, A ............................. 1599

Miyamoto, H..... 804, 830, 833, 834

Miyazawa, M ......................... 1100

Mizoguchi, Y............................ 1149

Mizuno, N ................................ 1441

Mnayer, L..................................... 324

Mo, JQ.................................. 1573

Moatamed, N........................ 236

Moch, H ................ 533, 705, 1648

Mody, DR...................... 975, 1065

Moffitt, R................................... 917

Mogal, A................................... 1261

Mohamed, A.............................. 764

Mohammed, MS ...................... 1696

Mohammed, RA....................... 246

Mohanty, A................................... 398

Mohseny, AB........... 75, 100, 1565

Mojica, WD.............................. 1698

Molavi, D ................................... 140

Molavi, DW ................................. 306

Molberg, K ................................. 946

Molberg, $\mathrm{KH}$
Molina, TM ................................ 426

Molina-Kirsch, H ....................... 1263

Molinaro, L .............................. 1508

Moline, T....................................269

Moline, V ............................. 1357

Mollejo, M ......... 1157, 1226, 1282

Moller, H.................................... 861

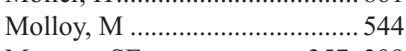

Monaco, SE.................... 357, 399

Monahan, J...................... 663, 1756

Mondaini, N .............................. 841

Mondul, AM.............................. 894

Monfulleda, M ......................... 739

Monfulleda, N ........................... 738

Montag, A............... 145, 187, 1098

Montalban, C........................... 1262

Monte, N .................................... 1056

Monteagudo, C................. 475, 479

Montes-Moreno, S ....... 1157, 1226,

$1262,1282,1283$

Montgomery, E ......... 35, 588, 636,

$671,835,1456$

Montgomery, K............. 1019, 1669

Montgomery, KD .................... 289

Montironi, R............ 729, 757, 812

$902,904,1649$

Montone, K ............................. 1147

Montone, KT...... 1137, 1138, 1744

Montpetit, A ............................... 39

Montserrat, N ........................ 1042

Monzo, M.................................... 1632

Monzon, FA..................... 875, 919,

$1183,1355,1690$

Moon, CM................................. 377

Moon, KC ...................................... 730

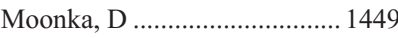

Moore, D ................................. 1340

Moore, DM .................................. 411

Moore, DT................................... 235

Moore, P....................................... 481

Moore, S..................................... 165

Moore, TL .................................. 21

Moorhead, J............................... 516

Moraes, NG................................ 280

Moran, CA..................... 1540, 1643

Morassi, F .............................. 1102

Moreira, A......... 1571, 1596, 1641

Moreira, RK ............................ 1437

Morel, P................................... 1433

Moreno, CS .................................. 836

Moreno, J ........................ 362, 670

Moreno-Bueno, G ...................... 255

Morente, MM ........................ 1288

Morgan, EA............................ 76, 247

Morgan, M ....................... 443, 913

Morgan, MJ................................... 1305

Morgenstern, S ......................... 1586

Mori, N.................................... 1320

Moriarty, A …............................. 359

Morice, WG ........ 1175, 1184, 1222

Morikawa, T.............................. 630

Moritani, S .................................. 203

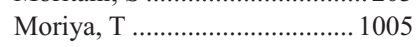

Moro-Sibilot, D......................... 1612

Morote, J .................................. 893

Morovic, A ................... 1263, 1264

Morris, DJ ................................... 915

Mortuza, S........................................ 1745

Mosher, R......................663, 1686,

1730,1756

Mosher, RM ............................. 1353

Moshkin, O ..................................... 1043

Moskaluk, A .............................. 1772

Moskowitz, A ........................... 1237

Moskowitz, C.......................... 1237
Mosquera, JM ............................ 77

Moss, B .................................... 1674

Mosse, CA............................... 1338

Mosunjac, MB................ 132, 1335

Mosunjac, MI.......................... 1335

Mothafar, F.............................. 161

Motiwala, N ................................ 837

Motoi, F.................................. 1463

Motoi, N............ 1595, 1596, 1624

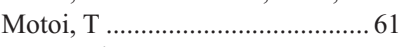

Motosugi, U ............................... 633

Moulton, CA ............................ 1453

Mounajjed, T............................ 1573

Mount, S..................................... 365

Mourra, N................................... 631

Mourtzinos, N ................. 838, 839

Mow, B....................................... 1312

Moyano, S ................................. 1112

Mozos, A ...................... 1265, 1332

Mroczek-Musulman, E ........... 1774

Msangi, G................................. 920

Mucci, L...................................... 760

Muci, T........................................ 228

Mueller, J ................................ 1601

Mueller-Hermelink, HK.......... 1205

Muenzer, JT............................... 1349

Muezzinoglu, B....................... 1044

Mulcahy, H.............. 568, 581, 614

Mulcahy, HE ............................. 572

Muller, S................................... 1139

Muller-Hermelink, K .............. 1263

Mulligan, AM........... 117, 184, 248

Mulligan, NJ ............................ 1088

Munkarah, A.............................. 936

Munkarah, AR .......................... 939

Munoz, J................................... 948

Munoz, N …........... 738, 739, 1026

Munson, P ................................ 1281

Murali, R ......................... 400, 470

Muralitharan, S ....................... 234

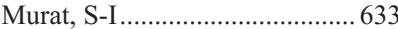

Murillo, A.................................. 260

Murphy, K................................. 1037

Murphy, L................................. 1080

Murray, M ................................. 1767

Murty, V ....................... 1244, 1321

Murty, VV .................................... 1314

Musumeci, F .............................. 346

Muto, MG ................................... 971

Mutter, GL.................................. 1023

Muzzafar, T .............................. 1260

Myohanen, T ........................... 1385

Myung, JK................... 1499, 1509

\section{$\mathbf{N}$}

$\mathrm{Na}, \mathrm{W}$........

600,632

Naber, SP $\quad 111,654$

Nabizada-Pace, F ............. 828, 914

Nachamkin, I........................... 1137

Nadal, A........................................ 855

Nadasdy, G...................... 1362, 1374

Nadasdy, GM .......................... 1382

Nadasdy, T............1362, 1374, 1382

Nadell, JM

Nadji, M .................... 253, 279, 779

Nafa, K........................ 1128, 1633

Nagai, T.................................. 1377

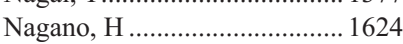

Nagata, K …...................... 242, 633

Naghashpour, M................ 485, 1337

Nagi, C ....................................... 206

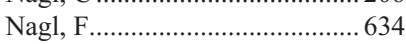

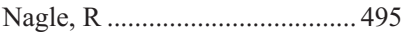

Nagler, A ...................................... 501

Nagori, S ................................... 364

Naini, BV ................................. 1438

Nair, V .................................... 1062

Naito, S ....................................... 900

Naito, Z ................................ 1778

Najafian, B ............................... 1378

Najarian, R ................................. 768

Najarian, RM............................... 635

Nakagawa, K................ 1689, 1697

Nakagawa, S ......................... 1030

Nakamura, S............................ 1320

Nakano, M.................................. 886

Nakashima, Y ............................. 58

Nakasono, M ............................... 657

Nakaya, T .................................... 630

Nakshatri, H ............................. 312

Nalesnik, MA .......................... 1454

Nalluri, S ................................... 1267

Nam, JH ..................................... 219

Nandi, I ...................................... 1183

Nanji, S ................................... 1453

Nannapaneni, S ....................... 1139

Naora, H................................... 1107

Narasimhan, S .......................... 249

Nart, D....................................... 1439

Nascimento, AF................. 76, 247

Nascimento, AG............. 69, 70, 71

Nash, B................................. 1666

Nasir, A.................. 149, 250, 518,

$519,593,1406,1708$

Nasir, NA ...... 518, 519, 1406, 1708

Nasr, MR ................................... 1266

Nassar, A ................ 150, 159, 251,

$252,938,1743$

Nassar, H............... 204, 288, 306 ,

1545,1554

Nassiri, M...................... 253, 1676

Natarajan, S................... 1546, 1610

Nath, I ..................................... 1345

Nathwani, B .................................. 1263

Natkunam, Y ................ 1249, 1257

Natrajan, R .............. 254, 255, 277

Naugler, CT............................. 1123

Navarro, A................................ 1632

Navarro, L ................................. 127

Navarro, PA............................... 1611

Navarro-Conde, P.................... 1282

Nawgiri, R................................ 942

Nayeemuddin, KM.............231, 257

Nazarian, RM............................. 476

Nazeer, HAT............................... 808

Nazeer, T ................. 808, 815, 1212

Necchi, A.................................... 734

Neder, L....................................... 1510

Nederlof, PM......................... 1646

Nedeva, T ................................... 1205

Nehhozina, T.............................. 326

Neimier, L ................................. 137

Nekhlyudov, L.................. 143, 144

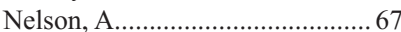

Nelson, BP ................................. 1318

Nelson, M..............................52, 89

Nelson, R......................................... 1059

Nepomuceno, E......................... 1543 
Neufeld, H.................................. 1225

Nevadunsky, NS......................... 971

Newman, KK ………………….... 845

Newton, K ………………..... 1259

Newton, KM …………………... 935

$\mathrm{Ng}, \mathrm{HK} \ldots \ldots \ldots \ldots \ldots \ldots \ldots \ldots \ldots \ldots \ldots \ldots \ldots . . .1503$

$\mathrm{Ng}, \mathrm{SB} \ldots \ldots \ldots \ldots \ldots \ldots \ldots \ldots \ldots \ldots \ldots \ldots \ldots . . .1312$

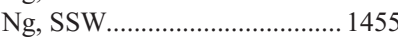

$\mathrm{Ng}, \mathrm{T} \ldots \ldots \ldots \ldots \ldots \ldots \ldots \ldots \ldots \ldots . . .256,1547$

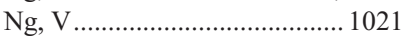

Nguyen, B ………………. 762, 857

Nguyen, BN …………………..... 761

Nguyen, CV ……………...257, 258

Nguyen, M ………….... 1202, 1245

Nguyen, PL ............................... 1240

Nguyen, T................................... 636

Nguyen, VT ................................ 1267

Ni, JS ...................................... 315

Nicastri, AD .................................909

Nichols, WS ………………....... 1004

Nicholson, RI ............................. 162

Nicol, K.................................... 1350

Nicolai, N..................... 734

Nicosia, RF …………………... 1379

Nicosia, SV ………........519, 1708

Nie, K …………………….... 1324

Nields, HM..................................... 21

Nielsen, GP ………………....59, 78

Nielsen, M.................................... 803

Niemeier, L................................... 264

Niemeier, LA ................................. 259, 846

Nikiforov, YE... 401, 402, 403, 517 , $526,527,541,1142$

Nikiforova, M

Nikiforova, MN................401, 402,

$403,526,527$

$541,592,1142,1594$

Ninomiya, H............................... 1623

Nisenbaum, H ………………..... 194

Nishikimi, N..............................901

Nishimori, I ................................... 85

Nishimura, H............................. 1286

Nishimura, R ………………..... 1003

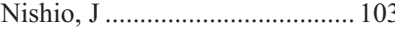

Nitta, H........................... 260, 1300

Niu, H.......................... 456, 1045

Niu, HL ....................................... 194

Niveiro, M..........110, 228, 261, 273

Noble, L ………...... 687, 871, 1622

Noe, M ……………………..... 732

Noel, P................................... 1254

Nofech-Mozes, S......262, 267, 334,

$965,986,995$

1043, 1046, 1047, 1058

$1071,1072,1091,1197$

Noffsinger, A............. 644, 645, 684, $685,686,1473$

Noh, JH ........................................ 667

Nolan, G...................................... 430

Nolan, NJ ...................... 1440, 1461

Nomura, K................................. 1624

Nonaka, D47, 442, 847, 1586, 1701

Nonogaki, S................................. 550

Nordio, B............................... 1588

Noreen, S.......................... 828, 914

Noronha, YS.................................263

Norton, L ……………………..... 326

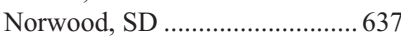

Nose, V.........................502, 523

$524,528,124$

Nosho, K .......................... 578, 638

$641,642,643$

Noth, I ............................................ 1592

Notohara, K.......... 1429, 1435, 1441

Notterman, D................................. 666
Novak, AJ.................................. 1279

Novak, L..................................... 1095

Novoa-Takara, L ...................... 1183

Nozaka, H ………………….... 1482

Nucci, M ……………………. 1040

Nucera, C .................................. 528

Nunes, FG …………………... 1338

Nuzzo, E...……………………..... 365

\section{O}

Oakley, GJ.

1131

O'Bannon, AB..................373, 1625

Obermeyer, J ...............................266

O'Brien, MJ .................................. 688

O'Brien, TE........................................... 1284

Ochoa, E................................... 1454

Ochoa, EO.................................. 1442

O'Connell, F ..................................22

O'Connell, PR.................... 568, 581

O'Connor, A .................................. 755

O'Connor, L ....................................

O'Connor, S ................................. 180

O'Connor, SM..............................264

O'Donnell, RJ ………….............. 48, 41

O'Donoghue, D......... 568, 581, 614

O'Dorisio, MS ................................ 816

O'Dorisio, TM ………………........ 816

O'Driscoll, L ............................. 212

Odze, RD.......... 567, 582, 583, 598, $607,608,640,668,669$

Oertel, J ........................................ 848

Ogane, N .................................. 1100

Ogawa, F........633, 639, 640, 1010

Ogino, S .... 578, 638, 641, 642, 643

Oh, B-K................................. 1421

Oh, HJ ……………………............. 606

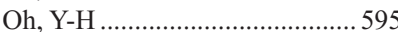

O'Hara, CJ ...................... 1638, 1777

Ohashi, K .................................1599

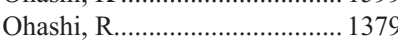

Ohba, T................................... 1623

Ohori, NP .......... 401, 402, 403, 527

Ohori, PN …………………...... 1593

Ohsie, S ..................................... 1354

Ohsie, SJ ........................................ 529

Okamoto, OK ..................695, 1552

O Keane, JC …………………..... 1088

Okumura, M..............................1603

Olawaiye, A A......................................... 1084

Olczyk, J ........................253, 1676

Old, LJ............................695, 1552

Oldridge, D ............................... 1699

O'Leary, J..........................513, 521

O'Leary, JJ ........ 358, 511, 714, 937 , $970,976,1549,1715$

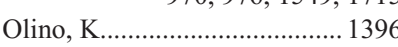

Oliva, E ................. 961, 984, 1009,

1010, 1026, 1038

$1039,1075,1084$

Oliva, I .................................... 1443

Oliva, IV ...................................... 1444

Oliveira, AM . 69, 70, 71, 450, 1040

Oliveira, RV .............................. 716

Oliveira, VC …ㄴ..

Olobatuyi, F ………………….... 1649

Ologun, BA …………………...... 1048

Olson, PR …………………….... 1761

Olson, SJ ........................... 42, 88, 96

Olson, SM ................................ 1140

Olteanu, H............1206, 1309, 1746

O'Malley, FP............. 117, 184, 248

Omar, I............................................. 43

Omeroglu, G ..............................267

Onate, JM.................................... 811
O'Neil, M.................................... 265 Onuma, K............... 161, 1049, 1093 Opekun, AR …………………......573 Oprea, G................................... 229

Oprea-Ilies, GM ………...... 131, 404 Orazi, A ...................................... 1180 Ordi, J................... 974, 1026, 1112 O'Regan, R ...................................131 O'Reilly, KC ………………........ 384

Orenstein, JM ............................ 1170 Orezzoli, JP .................. 1009, 108

Orlando, C................................. 1150

Ormsby, A ........................333, 1449

Ormsby, AH .............................. 1419

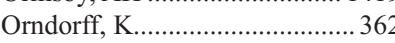

Ornstein, DK ………………….... 900

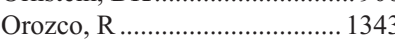

Orr, R ……………………….... 443

Orr, RK ……………………… 1305

Ortiz, P ..................................... 1282

Oryschak, A................................ 878

Osamura, RY .....................205, 536

$887,1734,1754$

O'Shea, D................................... 1659

Osman, JM ................................. 1779

Ostrom, L .......................663, 1756

OSullivan, B............................ 1135

O'Sullivan, J ............. 568, 572, 581

Osunkoya, A.................................. 844

Osunkoya, AO........... 705, 787, 807 , $814,836,849,869,917$

Ota, S...................................... 1030

Otis, CN ......................................985

O'Toole, S ........................970,976

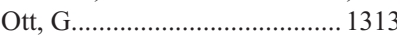

Ouyang, J ............................................. 1280

Owen, D .................................. 1455

Owen, DA …………………….... 1455

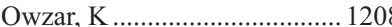

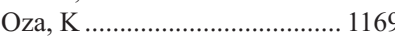

Ozcan, A.................................... 1771

Ozdamar, A................................ 1735

Ozen, S...………………….... 1380

Ozgun, MT ……………………… 1

Ozonoff, A................................. 1377

Ozturk, F .

...... 1

\section{$\mathbf{P}$}

Pacak, K …………………….... 1779

Padmanabhan, V ..... 405, 410, 1755

Padmore, R............................. 1172

Paessler, M.............................. 1198

Page, A ………………................. 462

Page, DL...................................... 129

Paglierani, M.............................. 842

Pagliuso, J ..................................... 400

Pai, R...................................... 684

Pai, RK ……………….....644, 645

Paik, SS ............................600, 632

Paik, Y-A ………....... 993

Paish, EC.........................2239, 246

Pal, TR _....1050

Palacios, J.........................254, 255

Palanisamy, N ... 773, 774, 824, 853

Palazzo, J................................. 1765

Palazzo, JP ................................. 943

Palekar, A ………………………. 79

Palescandolo, E ................................... 1699

Pallares, J ....……………….....1075

Palmisano, E .............................. 1458

Pambuccian, SE ………... 131, 370

404,413

Pan, CC ………………………..... 840

Pan, C-C................................... 821

Pan, Q...................................... 1747

Panarelli, NC................... 646, 1445

Panchananthan, R............. 693, 863

Paner, GP.................. 702, 712, 721

$770,776,850,851$

Panizo, A ……………... 1496, 1626

Pankey, GA …………………... 1330

Pantanowitz, L ………………....... 985

Pantuck, A ……………….......732

Papachristou, DJ .............................. 79

Papalas, JA …………………....... 477

Papathanasiou, A....................... 1011

Papin, JF ................................... 1339

Parada, C …………………..... 1557

Paragh, G.......................................... 318

Parangi, S ..................... 528, 1559

Parast, MM................................. 1575

Pardal, F _........................ 1506

Pardanani, A .............................. 1240

Pardo, J...……………………1626

Paredes, D …………………….... 340

Parisi, A..................................... 1711

Park, B

Park, BH................. 802, 852, 1000

Park, CK.............. 54, 602, 659, 667

Park, DC...................................... 794

Park, DY....................603, 604, 615

Park, HR.................................... 102

Park, J...................132, 1335, 1391

Park, JB .................................... 595

Park, JM …………………….......... 1133

Park, JS _.......................... 1001

Park, JY ......................377, 636, 647

Park, S ............................. 1020

Park, SH ...... 525, 1051, 1499, 1509

Park, SY ……………….... 525, 648

Park, WS ……………………..... 852

Park, YK …………………………......... 102

Park, Y-K....................................... 56

Park, YN.................................... 1421

Park, YW .................................... 874

Parkash, V …………….....679, 724,

$1024,1652,1658$

Parker, KJ ................................. 1352

Parker, N ……………………......... 353

Parker, R................................ 1007

Parker, SS ................................... 1164

Parkos, C …………………..... 570

Parks, GE ……………………..... 406

Parks, WT..................................... 264

Parmigiani, G ............................ 1396

Parra, ER ……………………..... 1627

Parrilla Castellar, ER ................. 1052

Parrish, T....................................... 394

Parsons, DW..............................1396

Partridge, D ................................. 1511

Parwani, A ............................... 429

Parwani, AV ............. 746, 750, 782,

$846,927,928,1355$

$1356,1358,1716,1731$

Pasha, T........................... 456, 718

$1147,1231,1434$

Pasha, TL............. 40, 45, 332, 1045

Pasini, B …………………...... 240

Pasqualucci, L...…………….... 1170 
Patel, KU Patel, MC ....................... 441, 1270

Patel, NP..................................... 557

Patel, R ……………………....... 438

Patel, S ................................... 532

Pathrose, P................................ 1618

Patil, DT .................. 650, 651, 1628

Patil, N .................................. 1618

Patil, NM...................... 411, 1340

Patsouris, E ............................. 1158

Patterson, KV ................................ 10

Patterson, P................................ 1311

Patwardhan, GA ......................... 1695

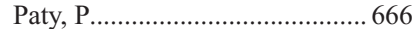

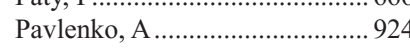

Pavlovich, C............................... 699

Pavlovitz, B ................................ 922

Pawlik, TM....................................596

Paya, A …................. 228, 286, 546

Payne, JI ........................................ 275

Pea, M ..................... 820, 821, 840

Peacock, G ............................. 1645

Peake, D ...................................... 90

Pearson, S.................................. 1166

Pearson, V .................................... 364

Pease, E ...................................... 1411

Pectasides, E ............................. 1667

Peddinti, R................................ 1519

Pedigo, MA …………………..... 376

Peer, G....................................... 1339

Pefanis, G.................................... 747

Peg, V ..............................269, 1557

Pei, Y....................................... 1053

Peiper, SC....................... 837, 1703

Peiro, G ................... 110, 228, 261,

$270,273,286$

Peker, D.......................... 271, 1271

Pellicer, A .......................................921

Pellin, A.............................. 475, 479

Pellin-Carcelen, A ............. 475, 479

Pellitteri, PK............................... 388

Pelosi, G.................................... 1629

Pelzer, L ……………………....... 674

Pena, J ....................................... 1042

Penault-Llorca, F....... 125, 260, 281

Peng, SL ........................ 1054, 1082

Peng, Y...... 192, 193, 198, 921, 946

Peng, Z ...................366, 1119, 1144

Pennington, C............................. 1366

Peplonska, B …………………....2292

Pereira, TC …………….... 398, 418,

443, 508, 1726

Perelman, M............................ 1630

Perepletchikov, A ............................... 846

Perepletchikov, AM.................... 928

Peressotti, C .............................. 1772

Perez, L ...................................... 546

Perez, M .................................. 1272

Perez de Santos, AM ................... 492

Perez Guillermo, M.................. 1283

Perez-Atayde, AR …………….......... 353

Perez-Fernandez, E .................... 1034

Perez-Ordonez, B ......... 1135, 1141,

1151,1152

Perez-Villa, F ............................... 340

Perkin, SL.................................... 469

Perkins, GH................................. 169

Perkins, LA ……………………....... 406

Perkins, SL ................... 1221, 1252,

1268,1304

Perli, E............................................... 346

Perlman, E................................... 1568

Perner, S ........... 781, 853, 854, 885,

$925,1648,1674,1677$

Perou, CM....................... 130, 283
Perren, A...................................... 533 Perry, A.......... 133, 190, 1486, 1487 Perry, K ……………………....... 89 Persky, DO ..................................... 1237

Pesavento, K .............................. 1760

Pestano, GA.............................. 1762

Peter, A ....................................... 1209

Peter, M ........................................ 488

Peters, L ................................... 1450

Petersen, I........................... 57, 1596

Peterson, L .............................. 1194

Petit, A...……………………... 855

Petras, ML …………………..... 1748

Petrich, AM ………………........1769

Petrolla, AA.................................. 652

Petrovics, G...................... 764, 765

Petrozza, V ................................. 747

Petsas, T ....................... 1412, 1413

Pettay, J J........................260, 1762

Pettenati, M …………………..... 1161

Petti, CA...…………………... 1346

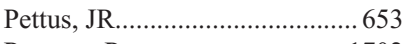

Pevsner, P.................................. 1702

Pezzella, F ................................. 1544

Pfeifer, J ...................................... 981

Pfeifer, JA..................................... 190

Pfeifer, JD ..........................304, 449,

$991,1006,1597$

Pfeifer, W .................................. 494

Pflueger, D ……….. 781, 853, 1677

Phadke, PA .....................654, 1522

Phan, DC …………....619, 655, 856

Phan, JH ..................................... 917

Phelan, SM ................................ 1659

Phillips, DGK ………….....272, 284

Piazzini, M ................................... 841

Picci, P ....................................... 98

Piccoli, A......................................... 1358

Picken, MM.............................. 1373

Piemonti, L.................................. 512

Pier, T ……………………...... 784

Pierce, AD ................................. 1273

Pierga, JY …………………….... 185

Pierron, G.................................... 185

Pilarski, RT................................. 186

Pilch, BZ ................................. 1148

Pilkington, L................................ 358

Pina, C..................................... 1677

Ping, L............................................ 113

Pinheiro, C …………………...... 1506

Pinkus, GS...................... 419, 1255

Pinnaduwage, D ................. 117, 248

Pinotti, G ……………………... 1306

Pinto, AP ...................... 1055, 1056

Pinto, M......................1652, 1658

Pinto, P ...................................... 907

Pipas, JM .................................. 1719

Piris, A ..........................................4 476

Piris, M..................................... 1226

Piris, MA............. 1157, 1262, 1281,

$1282,1283,1288$

Pisters, LL …………………....... 707

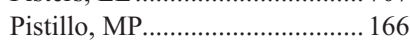

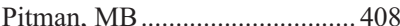

Pittaluga, S .............................. 1291

Piva, L ........................................ 734

Pizzolitto, S............. 163, 451, 1488

Placidi, C................................ 1306

Placido, JB ............................... 1341

Planas, S...................................... 893

Planelles, M....................... 110, 273

Plante, M................................... 953

Plantier, F .................................... 881

Platz, E ………………………..... 895

Platz, EA ……………………...... 894
Plaza, JA............................ 446, 480 Plesec, TP.................................... 656

Pletneva, M ................................. 490

Pliner, L..................................... 1311

Plonczak, AM................................ 343

Plotkin, A...................... 1057, 1058

Podratz, KC............................. 1041

Pohlman, B.................................. 1214

Polakiewicz, R ......................165

Polakiewicz, RD ...................... 1711

Pollack, JR .................................957

Pollett, A................................... 1453

Pollock, RE ........................... 64, 81

Polski, JM ................................ 1274

Pomeroy, C.................................... 116

Pommepuy, IS ……………….... 1490

Ponce-Rodriguez, A .................. 1113

Ponnazhagan, S ......................... 438

Ponnusamy, MP........................... 1059

Pons, B ………………………..... 269

Pons, C ………………... 948, 1042

Pontecorvi, A.............................528

Pontes, J ....................................... 695

Poon, WS …………………....... 1503

Poppiti, RJ......................271, 1725

Porrello, A ..................................... 528

Post, JC ……………………...... 601

Potash, MK ………………….... 1631

Poterrucha, J............................. 1480

Potthoff, SA..............................1385

Pourmand, N ................................ 548

Powell, CB .................... 1012, 1029

Powell, SZ................................... 1487

Powers, CN ………….....386, 1693

Powers, MP …………………...... 80

Pozo, L ……………………….... 459

Prasad, A …………………….....495

Prasad, AR...………………….............. 478

Prasad, M ................................... 237

Prasad, V .................. 19, 1350, 1446

Prat, J _.................... 948, 949 ,

Prayson, NF............................ 1512

Prayson, RA .................... 439, 1487,

1504,1512

Prchal, JT …………………...... 1304

Preobrazhensky, SN ..................... 1346

Prichard, JW ............................. 1025

Prieto, VG .......................... 446, 480

Prinz, C ……………………...... 590

Priolo, C ……………..... 528, 1699

Prokhorova, IN ............................ 707

Prowse, D ....………………...... 715

Proytcheva, MA ......................... 1318

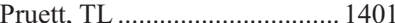

Pryor, JG ........................................ 472

Przybycin, CG..........................1060

Pucevich, BE ………………….... 1188

Puduvalli, V ............................. 1492

Puig, X ………………………..... 660

Pulitzer, MP......................................... 481

Purdom, MS ....……………....2 274

Purgina, BM .............. 761, 762, 857

Puri, A ……………………........ 82

Pusztai, L............................................ 1533

Putnam, A.................................... 1566

\section{Q}

Qi, C.......... 1172, 1223, 1247, 1275 Qian, J......... 694, 1487, 1539, 1548 Qian, ZR..........................538, 657 Qiao, YL.................................... 1026 Qin, J ....................................... 1080 Qin, L ............................ 1409, 1464

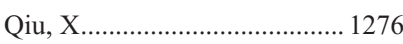
Qu, Z ...........................1352, 1749

Quackenbush, J ................. 149, 250

Quah, TC ................................... 1312

Quale, C …………………….... 116

Quann, P...................................... 858

Quayle, K ................................ 1495

Quddus, MR .............................. 331, 394,

$1048,1054,1061$

Quek, ML ..................379, 702, 770

Quek, SEC................................ 1312

Quer, A ............................ 984, 1010

Quera, A ..................................... 1632

Quesenberry, C................... 143, 144

Quick, CM..........619, 655, 748, 859

Quilodran, S .............................. 670

Quinlisk, FM ............................... 405, 410

Quinn, A ................................... 182

Quint, W.................. 738, 739, 1026

Quintal, MM ………………....... 860

Quintanilla-Martinez, L ........... 1298

Qureshi, AA.................................. 496

Qureshi, MN ............................... 940

$\mathbf{R}$

$\mathrm{Ra}, \mathrm{SH}$.

482,1447

Raab, SS...

$506,509,1654$

$1660,1663,1665$

Rabban, J................................. 128

Rabban, JT ..... 156, 177, 969, 1012, $1029,1035,1036$

Racke, F ………………........ 1208

Rad, MTS ..................................1224

Rad, SMT .................................. 1253

Rademaker, A............................. 1628

Radio, SJ ………………………15

Radu, OM..................................9927

Raffeld, M..................... 1291, 1298

Ragazzi, M .............................. 277

Raghunath, P ............................ 1198

Raghunath, PN ......................... 1189

Raghunathan, A.......................... 1513

Ragsdale, BD ............................... 470

Rahimi, K................................ 1062

Rahman, MM ............................ 538, 657

Rahman, SS ............................. 1342

Raho, C ...................................... 952

Raich, R................................1728

Rainey, PM............................... 1750

Raitman, I..................................... 248

Rajab, R........................................... 861

Rajan, P .....................................233

Rajan, PB ........................ 266, 305

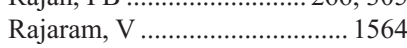

Rajendran, V ..................1119, 1144

Rajput, A ...................................... 133

Raju, M ..................................... 478

Raju, U ............................ 137, 333

Rakosy, Z …………………...... 318

Ramachandran, R..................... 1448

Ramalingam, P..........530, 796, 837

Ramesh, KH........................... 1269

Ramirez, J ................................ 340, 1632

Ramon y Cajal, S ............. 269, 893 ,

Ramos, D $\quad 1557,1688$

Ramos, S .................................. 253 
Rao, R

NM

Raoufi, M ....................... 494, 1449

Raparia, K .................... 1380, 1450

Rapoport, AP ........................... 1325

Rashid, A.......................... 575, 625

Rashid, AR ...................... 584, 626

Rasmussen, SL ........................... 589

Raso, MG ................................. 1643

Rassidakis, GZ .... 1158, 1186, 1245

Rasty, G................. 409, 997, 1057

Ratech, H............ 1156, 1269, 1747

Rauchman, M........................ 1295

Raut, CP ........................... 76, 247

Ravindranath, L.......................... 765

Ravulapalli, S ........................... 900

Rawson, RAM........................... 1063

Raymond, AK .............................. 99

Raymond, D ............................. 1590

Raynal, V ................................. 185

Raynaud, C.............................. 1612

Rayson, D................................ 275

Rayter, S.................................... 255

Raza, AS ..................................... 263

Rea, F ................................... 1588

Recavarren, R............................. 361

Reddy, P ................................... 276

Reddy, R................................. 1434

Reddy, V.................................. 1578

Reddy, VB................................. 1341

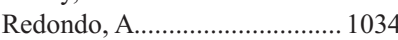

Reece, D ...................... 1172, 1275

Reed, RC ................................... 1750

Reed, SD ................................... 935

Regauer, S .................................... 1064

Regenstein, F.......................... 1403

Rehana, J ................................ 177

Rehrauer, WM........................... 1262

Reich, H ................................. 1375

Reichard, K ............................. 1196

Reichard, KK …............. 1273, 1662

Reid, BJ.................................. 567

Reid-Nicholson, M.................... 837

Reid-Nicholson, MD......... 530, 796

Reilly, SD ....................................... 33

Reis, I ............................. 779, 780

Reis, RM .................................. 1506

Reis, ST ................................... 1694

Reisenbichler, ES ..................... 278

Reis-Filho, JS ... 175, 240, 244, 254 $255,277,322,447,1506$

Reith, JD ........................... 52, 103

Rekhi, B ..................................... 82

Rekhtman, N ................... 800, 1633

Relliga, P ............................. 344

Remick, D ................................. 1610

Remick, DG ........ 1334, 1530, 1546

Remmenga, S ............................ 980

Remotti, HE ............................. 1478

Remsen, T ................................ 1702

Remstein, ED .... 1163, 1222, 1242, $1277,1278,1279,1317$

Remzi, F .......................... 569, 656

Ren, L...................................... 1315

Ren, Y........................................ 1569

Rena, O ................................... 1603

Renaud, M-C................................ 953

Rendi, M .................................... 410

Repertinger, S............................. 173

Resetkova, E ..................... 258, 1492

Resnick, KE ............................. 947

Resnick, M ............................... 1537

Resnick, MB ... 687, 871, 915, 1622

Reuben, J........................ 221, 222

Reubi, JC ................................. 610
Reuter, V .................................. 861 Reuter, VE..............696, 759, 792 $864,865,892,1752$

Reyes, C .......................... 253, 279

Reynolds, C.................... 118, 316

Reynolds, CA............................. 25

Reynolds, J............................ 1618

Reynolds, JP...

411,544

$693,863,1340$

Rezaei, MK ..................... 838, 839

Rezai, T ..................................... 313

Rezania, D............................... 485

Rhaleb, N-E.............................. 1385

Rho, JH …................................. 1700

Ribeiro, JMFP ............................ 1627

Ricarte-Filho, J................ 531, 532

Ricci, Jr, A................ 202, 211, 324

Rice, MS .....

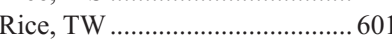

Richards, DL ............................. 1065

Richards, WG.......................... 1601

Richardson, TJ ................................. 33

Richter, L..................................... 507

Richter, P....................................... 57

Rickman, DS .................. 853, 854

1674,1677

Riddell, RH ............................... 573

Ridder, R .................................... 962

Riely, GJ................................... 1633

Riley-Portuges, CJ ................... 1083

Rimm, DL ..................... 1667, 1717

Rimsza, LM.............................. 1300

Ring, M .................................... 937

Rink, RC ................................... 884

Rioux-Leclercq, N.................... 751

Risendal, B................................. 197

Risley-Penn, AD .......................... 415

Rivera, M ......................... 531, 532

Rizzo, EJ .................................... 295

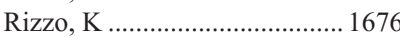

Rizzo, KA.................................. 1227

Rizzo, M.................................... 132

Ro, JY............. 682, 769, 788, 802

$852,874,875,919$

$1130,1450,1690$

Roa, JC ..................................... 1392

Robbins, L................................ 1295

Roberson, S ............................... 241

Robert, SA.................................. 1490

Robertson, J................... 411, 1340

Robetorye, RS ...............328, 1675 ,

1696,1751

Robinson, SJ ...............................1111

Robstad, KA......................................... 658

Rocco, GL ................................. 376

Rocha, RM .................................. 280

Rochaix, P ................................. 227

Roche, H ...................... 227, 1692

Roche, N ................................ 1066

Rock, J........................................ 83

Rock, KL....................... 1494, 1542

Rodas, O.................................. 1540

Roden, AC ................................. 1634

Rodenas, A ................................... 109

Rodig, S....................................... 1583

Rodig, SJ ... 1280, 1319, 1553, 1635

Rodon, N ..................................... 660

Rodriguez, EF ….............................. 1514

Rodriguez, ER........... 345, 348, 349

Rodriguez, FJ .................. 1489, 1514

Rodriguez, M ........................ 1563

Rodriguez, ME ........................... 1288

Rodriguez, MH ....................... 1262

Rodriguez de Cordoba, S ........ 1557

Rodriguez-Justo, M.................. 1281
Rodriguez-Peralto, JL ............. 1282

Rodriguez-Pinilla, SM ..... 254, 255 $1262,1282,1283$

Rodriguez-Urrego, PA............. 1752

Roehl, H ..................................... 101

Roehrl, MH .............................. 1700

Roepmann, P ............................. 287

Rofsky, N .......................... 753, 767

Roger, L.................................... 1692

Roger, P.................................... 281

Rogerio, F ….............................. 716

Rogers, HJ................................. 1284

Rogers, LW ............................... 20

Roh, JH …................................... 659

Roh, MH ..............412, 1067, 1076

Roh, MS .............................. 1636

Rohan, SM ................. 792, 864, 865

Rohwedder, A............................. 455

Rojas-Torres, ME ........................ 108

Rollins-Raval, MA.................. 1068

Roma, AA.................................. 1069

Romagosa, V .............................. 1167

Roman, R ................................... 660

Roman-Roman, S......................... 185

Romans, A................................. 626

Romaschin, AD .......................... 918

Romeo, S....................... 75, 84, 85

Romond, EH ........................... 274

Roncador, G .................. 1157, 1226

1281,1282

Roncella, S ............................... 166

Roncero, M ............................... 487

Ronnett, BM........ 1037, 1060, 1078

Root, RM.................................. 1743

Rose, AG ........................... 6, 9, 13

Rosen, B................................. 1062

Rosen, C.................................. 1480

Rosen, CB .................................. 1386

Rosen, DG............. 950, 963, 1107

Rosenberg, A ................................ 84

Rosenberg, AE .................... 59, 78

1010,1039

Rosenberg, C............................ 1685

Rosenberg, CL.......................... 1680

Rosenfeld, N ................... 763, 1701

Rosenthal, N.......... 1266

Rosenwald, A ........................... 1313

Rosenwald, S....... 1586, 1630, 1701

Rosenwald., IB ......................... 1236

Roses, R ...................................... 194

Ross, C ..................................... 468

Ross, HM ................................ 145

Ross, JS ........... 208, 282, 283, 300, $301,467,565,658$ $789,808,815$

$1212,1614,1615$

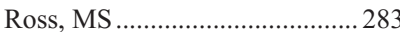

Rossi, S ........................... 661, 675

Rotblat, B .............................. 1547

Roth, A ….................................. 1433

Roue, G ...................................... 1265

Rougemont, AL ............................. 39

Roullet, MR.................................... 1231

Rouse, RV ................................ 1074

Rouzbahman, M..... 409, 997, 1057

Rouzier, R ...

Rowley, S ........................... 114, 115

Rowsell, EH ................................. 1176

Roy, M...................................... 953

Roy, R .......................................... 51

Roy Chowdhuri, S..................... 866

Royce, M.................................... 283

Rozenberg, F ............................. 488

$\mathrm{Ru}, \mathrm{K}$.......................................... 535

Ruan, J.................................. 1538

Rubbia-Brandt, L ................... 1433,

1452,1460

Rubens, DJ ............................. 1352

Rubin, BP ....................69, 94, 493

Rubin, MA........781, 853, 854, 885 $925,1648,1674,1677$

Rubinas, TC …............................ 662

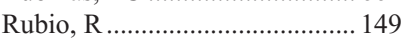

Rubio, V ...................................... 108

Ruddy, D .................................. 1756

Ruderman, NB ........................ 1524

Rudloff, U ................................ 326

Rudolph, T ............................... 533

Rudzki, B .................................... 165

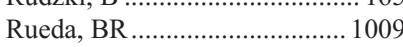

Ruiz, BI ................................. 1721

Ruiz, P..................................... 1103

Rumilla, KM ................... 483, 1070

Rusch, V...................... 1595, 1596

Rushing, EC ............................ 484

Rushing, EJ ..................... 46, 1483

Russell, AH ............................. 1084

Russell, P.............. 400

Russell, S..................................... 513

Russell, SM ............................... 511

Russo, P......................................... 696

Ruzinova, MB ......................... 1637

Ryan, P .................................. 1453

Ryan, RL ................................. 1763

Rybicki, LA................................................ 601

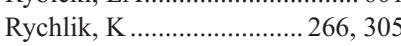

Rynearson, A ............................ 1514

Ryu, E........................................ 920

Ryu, JH..................................... 1640

Ryu, JK..................................... 1463

Ryu, MH...................................... 663

Ryu, M-H .................................. 1756

$\mathbf{S}$

Saab, S....................................... 908

Saab, ST …................................ 1285

Saad, AG ................................... 1670

Saad, R ...................................... 995

Saad, RS ............... 965, 986, 1043,

1046, 1047, 1058,

$1071,1072,1091$

Sabel, M................................... 325

Sabo, E ............................. 871, 915

Sabour, M....................................... 599

Sacks, N ..................................... 189

Sacristan, S............................... 1505

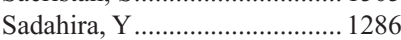

Saetta, M ................................. 1588

Saez, A......................................... 1262

Safar, B ............................... 552

Sagatys, EM …................. 485, 1337

Saha, MN ................................ 1287

Sahin, AA .................................... 169

Sahoo, S ............... 373, 1474, 1625

Said, A ........................................ 605

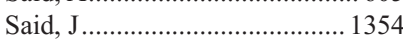

Saidman, S ......... 1363, 1364, 1369

Sailey, CJ.......................... 272, 284

Sait, S . ................................ 215 
Saleh, R

Salem, F .....

Salgia, R

1616,1642
161

Salhadar, A ...... 266, 305, 433, 1570

Salinitri, G................................. 747

Sall, A........................................... 351

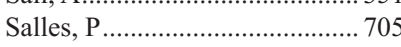

Salley, YM................................ 1549

Salman, H............................... 1295

Salvatore, J...................... 429, 846

Salvioni, R.................................. 734

Salwen, HR ............................. 1519

Samaan, S.............. 411, 544, 1340

Samadi, DB ....................... 828, 914

Samaniego, R .......................... 1505

Samara, M............................ 1550

Samaratunga, H................ 708, 867

Samarawardana, PN................ 1073

Samayoa, LM........... 136, 274, 382

Sams, SB ................................. 506

Sanati, S .............................. 218, 285

Sanchez, A................................. 990

Sanchez, B................................. 233

Sanchez, CA.................................567

Sanchez, G ............................. 1026

Sanchez-Beato, M................... 1157

Sanchez-Espiridion, B... 1262, 1288

Sanchez-Paya, J.......................... 270

Sanchez-Sosa, S ........................ 127

Sanchez-Tejada, L..... 110, 270, 286

Sanda, M ........................ 753, 767

Sanders, D .................... 1668, 1684

Sanders, M ................................ 486

Sanders, ME .............................. 296

Sanders, MG ............................. 1625

Sandquist, DW ........................ 1515

Sands, AK ................................. 1325

Sandstrom, DJ .......................... 1553

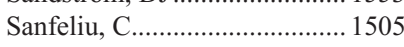

Sanga, S........................ 157, 1679

Sanger, W ................................ 1264

Sangoi, A ..................................... 1736

Sangoi, AR …................... 736, 822,

$823,859,1074$

Sanjuan, X..................................6 660

San-nohe, S .............................. 633

Sano, T ............................ 538, 657

Santacana, M.......................... 1075

Santos, J ......................... 979, 1008

Santos, SJ ....................................... 37

Santos-Briz, A ............................... 487

Santra, M................................. 1168

Sanyal, S ....................................... 25

Sanz, D....................................... 779

Sapgnoli, G .................................. 461

Sapino, A ..................................... 240

Saqi, A....................................... 421

Sarancone, S.............................. 168

Saravia, M................................. 1343

Sargent, RL .................. 1187, 1289

Sarker, S ...

Sarmiento, J................. 1398, 1399,

1443,1444

Sarmiento, JM ....... 672, 1392, 1465

Sarode, V....................................... 192

Sarode, VR ......................... 193, 198

Sarracino, D .................................. 313

Sarris, AH................................ 1186

Sartor, C ........................................ 661

Sasa, N ....................................... 901

Sasatomi, E ............................... 1454

Sasawaga, T ............................ 1026

Sastre-Garau, X................ 185, 488

Sato, K................................... 840
Sato, T .................................... 1482

Satoskar, A ................... 1362, 1374

Satoskar, AA............................. 1382

Sattar, HA.................................... 188

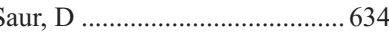

Sausville, EA............................. 1325

Savage, EC ................................. 1661

Savage, K ...................................... 244

Sawyer, JR ................................ 103

Saxe, DF................................... 1290

Saxena, R ................................ 1430

Saxton, J.................................. 1129

Saxton, RE ............................... 291

Sayage-Rabie, L.......................... 364

Sboner, A................................... 1677

Scardino, P ........................... 861

Scardino, PT............................... 892

Scepanovic, O ........................... 317

Schaefer, KL.................................. 37

Schaeffer, DF ........................... 1455

Scharpf, J................................. 1129

Scharre, K ........................... 395, 425

Schatz, P.................................. 727

Scheithauer, BW …................... 1489, 1514

Schemankewitz, E.................... 1333

Scher, HI ..................................... 892

Scherer, S ..................................... 25

Schernhammer, ES .................... 641

Schichman, SA............... 655, 1153

Schieffelim, JS ........................ 1330

Schiffhauer, L.......... 207, 311, 1725

Schiffhauer, LM ......................... 182

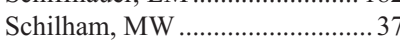

Schiller, CL ................................... 309

Schirmacher, P ................. 297, 298

Schlecht, NF............................ 1055

Schlette, E ................................ 1259

Schlette, EJ................................. 1186

Schlosshauer, PW...................... 956

Schlumbrecht, P ..................... 1097

Schmechel, SC .................4 413, 868

Schmid, J.................... 1727, 1763

Schmid, RM ..................... 590, 634

Schmid, S .................................... 533

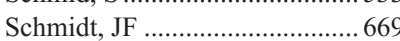

Schmidt, JL ................................ 868

Schmidt, RA............................... 183

Schmitt, AM ......................... 533

Schmitt, CL .............................. 1273

Schmitz, BA .................................. 413

Schnadig, V ............................... 942

Schnadig, VJ ............................ 1344

Schned, AR .......... 405, 1092, 1739

Schneeberger, EE ...................... 1369

Schneider, AG ........................... 736

Schneider, CM ............................... 26

Schneider, F ............................... 414

Schneider, G............................. 634

Schneider, J ................................ 590

Schniederjan, MJ..................... 1359,

1500,1516

Schniederjan, SD ............869, 1290

Schnitt, S ......................... 141, 142,

$143,144,320$

Schoedel, KE..................... 402, 403

Schoenfield, L .......................... 1528

Scholtz, G................................... 394

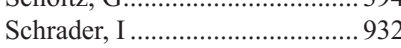

Schrager, JA ................................ 1291

Schreiber, ZJ ................................. 1050

Schubert, JM .............................. 385

Schuerch, C .................................. 388

Schulick, R.................. 1456, 1458

Schulick, RD ................ 1396, 1463

Schulte, S ............................... 1076
Schultz, AM .............................. 415

Schultz, DS …........................... 1294

Schultz, L .................................... 716

Schulz, S .................................... 943

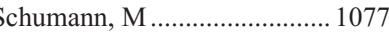

Schur, BC ……........................... 1746

Schutz, SN................................. 295

Schwab, MC ................................... 295

Schwartz, AM .......... 545, 838, 839

Schwartz, EJ............................ 1074

Schwartz, HS.................. 42, 88, 96

Schwartz, M ............................ 359

Schwartz, MR .......232, 1065, 1450

Schwock, J .............................. 1141

Schworer, CM ............................ 870

Sciallis, AP .................................. 27

Sciandra, K................................ 871

Sciot, R....................... 84, 97, 100

Sciulli, CM.............................. 928

Scognamiglio, T ................4 416, 428

Scollard, DM............................. 1345

Scolyer, RA ................................. 470

Scopa, C ..................................... 691

Scordi-Bello, IA .............................. 28

Scotlandi, K ….......................... 37

Scott, G ...................................... 499

Scott, GA.................................... 472

Scott, KM ..... 507

Scott, L ........................................ 572

Scrape, SR ................................. 872

Scudamore, CH .......................... 1455

Scudiere, JR ................... 636, 1456

Seager, A ................................... 1702

Sebastiani, M............................. 346

Sebastiani, P................. 1680, 1685

Sebo, T .................................... 920

Seeger, K................................... 1209

Seegmiller, AC …........................... 1292

Seery, PP .................................... 295

Seethala, RR............401, 402, 403,

$527,1127,1128$

$1131,1142,1143,1152$

Seetharam, M .......................... 1315

Segala, D ................ 722, 723, 821

Segui, J..... 110, 228, 261, 273, 286

Seidler, B................................... 634

Seidman, JD .................. 1013, 1078

Seigne, JD ................................... 405

Seijo, LM .................................... 391

Seiler, C...................................... 1681

Seitz, GA..................................... 287

Seldin, D ................................... 1200

Selegean, S ................................. 417

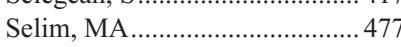

Selzner, N................................. 1387

Semenza, G ............................... 1560

Semidey, ME................................. 893

Semmel, D..................................1111

Semmel, DR ............................. 1638

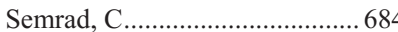

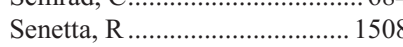

Senger, JL.................................... 210

SenGupta, S............................... 133

Senz, J ........................................ 932

Seo, JW ......................... 1613, 1650

Seo, K............................ 1315, 1316

Seol, J....................................... 1138

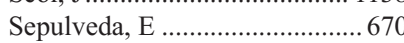

Sercia, L ..................................... 709

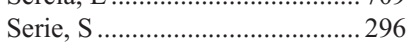

Serizawa, A .................... 205, 1754

Serra, S ......................... 489, 520, 534

Serrano, S................................ 1167

Seshan, S ................................... 1380

Sessa, F ................................... 1417

Sesterhenn, I................................ 890 Sesterhenn, IA... 764, 765, 777, 900

Sesterhenn, IS ........................... 791

Seth, A ....................................... 1046

Sethi, S ...................................... 1755

Sethu, S .................................... 1512

Setlur, SR …............................. 1677

Sever, C ......................................... 1218

Sevilla, DW ................... 1293, 1314

Sewrajsing, R ............................ 100

Sexton, MJ ...................................... 7

Seybt, TP ...................................... 29

Seydaoglu, G............................. 754

Sgroi, DC ..................................... 148

Shaco-Levy, R........................... 1461

Shafer, JA ............................... 1272

Shafizadeh, N ................... 664, 665

Shah, AA ….............................. 1144

Shah, JP...................................... 532

Shah, R .................................. 926

Shah, RB ........ 703, 773, 774, 824 $826,873,911,912$

Shah, S ............471

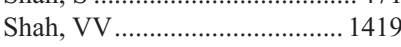

Shaha, A .................................... 532

Shahbahrami, B...................... 1724

Shak, S .....................114, 115, 116

Shalev, I...................................... 1387

Shalhav, A .................................. 805

Shamanna, R ............................. 333

Shamanna, RK ......................... 1294

Shang, P ................................. 1292

Shapiro, CL .................................. 186

Shapiro, R ............................... 1329

Sharafeldeen, B .......................... 288

Share, SM......... 418, 443, 508, 535

Sharer, LR ................................. 1493

Sharkey, FE ..................... 1079, 1656

Sharma, G .............................. 1513

Sharma, M..................................... 289

Sharma, R............... 490, 785, 786,

$844,1484,1587$

Sharma, S ..................... 796, 1703

Sharma, SG .......... 856, 1366, 1780

Shatat, L …................................. 1295

Shaw, AJ......................... 290, 1296

Shaw, PA .......................... 1062, 1704

Shayne, M .................................. 311

She, RC ..................................... 1346

Shea, CR ................................... 491

Sheahan, K...... 568, 581, 614, 1659

Shedden, K............................... 1372

Sheehan, CB ............................... 300

Sheehan, CE ..... 208, 282, 300, 301, $467,565,658,789,808$, $815,1212,1614,1615$

Sheehan, GM..................... 208, 789

Sheehan, K ................................ 572

Sheibani, K................................... 1647

Sheibani, S ............................... 1647

Sheils, O................................

$714,970,976,1549$

Sheils, OM ............................... 511

Shen, B .............................. 569, 656

Shen, D....................................... 291 
Sheppard, MN ... 339, 341, 343, 347

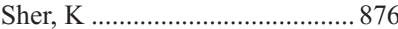
Sherer, C............................ 429, 846 Sherman, ME …………………....292

Sherr, DH ................................. 249 Sherwood, NT ........... 418, 508, 535

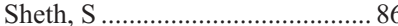
Shetuni, BB ……………………... 293 Shi, C.......................1457, 1458 Shi, J......................388, 420, 1025 Shi, W................................... 1135

Shi, Y..................................... 466 Shia, J............ 666, 677, 1080, 1391 Shidham, VB............................. 424 Shieh, W-J .................................. 1347

Shields, C .................................. 631

Shier, LR …………………….... 1256

Shih, I................................. 1014

Shih, IM ……....... 1015, 1016, 1101

Shih, K ……………………..... 978

Shih, LM …………………….... 999

Shikanov, S ………………….... 805

Shilo, K …………………....... 1619

Shim, JY ...................................... 993

Shim, Y.......................663, 1756

Shima, K ........... 578, 638, 642, 643

Shimao, D ................................. 203

Shimizu, M.......................633, 1100

Shimizu, Y...…………………........ 633

Shimoda, T ................................... 74

Shin, DH …………....603, 604, 615

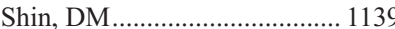

Shin, JH................................... 1081

Shin, SH ............................. 1420

Shinoda-Matsuoka, H .............. 1757

Shinozaki, A ................................630

Shintaku, P ............................... 236

Shinya, F ................................. 1328

Shipp, MA ………………….... 1280

Shiu, KK .........................240, 254

Shiue, YL ……………………..... 53

Shively, SB................................... 941

Sholl, LM ………………... 87, 1639

Shree, S .................................... 619

Shrestha, B ................................ 1640

Shroyer, KR ………………...... 1073

Shumaker, NR ……………….... 1662

Shunkwiler, SM …………….... 1579

Siadat, F ……………….... 409, 997

Sica, G.................................... 1641

Sicinska, E..................... 1553, 1699

Sidawy, MK ………………….... 330

Siddiqui, J .................................... 911

Siddiqui, MA................................. 294

Siddiqui, MT _..............159, 378

Siddiqui, S................................ 1667

Sidhu, D ………………... 877, 878

Sidhu, G ……………………..... 303

Sidiropoulos, N ………………....295

Siegal, G...................................... 438

Siegal, GP....................................... 36

Siegel, CS................................... 612

Sigal-Zafrani, B............................ 185

Signoretti, S...................... 768, 910

Silva, A ................................... 1506

Silva, CAM …………….... 717, 860

Silva, E...........................983, 1063

Silva, EG ........................ 1004, 1027 ,

1069, 1096, 1099

Silvas, E ……………………............ 666

Silver, S.................................... 1170

Silverberg, SG .......................... 1005

Silverman, JF ............ 398, 418, 432,

$508,535,1091$

$1561,1726,1770$
Silverman, JS

Silverman, SG

Sim, HG …………………….... 899

Simmerman, K .................. 757, 925

Simoes, K........................ 695, 1552

Simon, RA.................... 1054, 1082

Simons, CM ………………....... 816

Simpson, JF ........................................... 296

Simpson, S ............................... 293

Simsek, G................................. 1735

Simsir, A................................... 431

Singh, A A.................................. 1528

Singh, B ....................................22

Singh, H ................................. 1716

Singh, M........................................ 197

Singh, RR ..............626, 1230, 1259

Singh, VM .................................. 1083

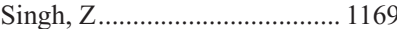

Singhi, AD................................. 1145

Sinn, HP ........................... 297, 298

Sioletic, S ..................... 1084, 1102

Sircar, K .......... 742, 862, 876, 1674

Sireci, AN .................................. 421

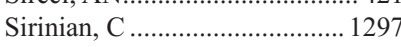

Sirintrapun, SJ .......................... 1359

Sirko-Osadsa, DA ......................... 597

Sirois, K ……………………... 1575

Sis, B ..................................... 1705

Sitkovsky, M ............................. 1530

Sitterding, SM ……………….... 1642

Siu, KWM ..................................... 918

Siu, MSK …………………...... 1106

Sivagnanalingam, U....... 1749, 1749

Sivarajah, K...………………... 1459

Siziopikou, KP ………………..... 299

Skaf, J...................................... 1406

Skeen, V ……………………... 1340

Skeen, VL..................................... 411

Skinner, K ......... 207, 307, 311, 319

Skipper, BJ ................................ 1273

Sledge, GW …………….... 114, 115

Slisz, J ...................................... 1686

Slodkowska, E...................282, 283

Slodkowska, EA..................300, 301

Slominski, A.................................... 467

Slotta-Huspenina, J ................... 1298

Smit, EF …………………….... 1646

Smith, AK ……………..... 879, 891

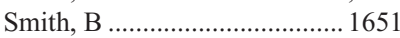

Smith, BL ..................................... 196

Smith, D …………………….... 975

Smith, DP ................................... 244

Smith, KD …………….....81, 1379

Smith, LM ..................... 1201, 1258

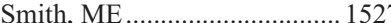

Smith, ML .................506, 509, 1663

Smith, MT ……………………... 744

Smith, R …………………….... 1337

Smith, RN ………….....1364, 1369

Smith, TW ………………….... 1494

Smolkin, ME ...............................728

Smoller, BR..... 453, 619, 655, 1125

Smulian, AG.................... 411, 1340

Smyrk, TC …................540, 1429

Smyth, P....................... 513, 521,

714. 970,521

Smyth, PC .................................... 511

Sneige, N.........................169, 181

$191,372,983$

Snijders, PJ............................... 1646

Snowden, CA …………………....... 302

Snuderl, M.................................. 1010

Snyder, C..................................... 563

Snyder, EL.................................. 1553
Soape, M ...

152, 280, 550

Soares, IC ……………..... 695, 1552

Sobel, ME ………………….... 1709

Sobin, LH............................. 62, 72

Sochacki, P............................... 494

Soderberg-Naucler, C................... 344

Soffer, E ……………………….... 564

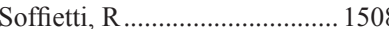

Sohani, AR ……...... 510, 1299, 1360

Soin, J.................................... 344

Sol, MY ……………........ 603, 615

Sola, JJ ……………..... 1496, 1626

Solis, LM................................... 1643

Solmos, G......................................... 299

Solomides, C ................................ 681

Soltermann, A.................................... 1648

Sommer, JB ……………………... 88

Somoza, R ..................... 1557, 1688

Son, $\mathrm{CH}$.................................... 1636

Sondak, VK ................................. 471

Sondej, T ..................................... 770

Song, GA............................................ 604

Song, HJ .......................................... 667

Song, J............. 491, 805, 880, 1616

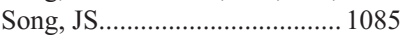

Song, SK …………………….... 586

Song, X ………………………... 303

Song, YS ........................................... 600

Sonzogni, A ................................ 1629

Soo Hoo, PT................................ 1777

Sood, A........................................ 939

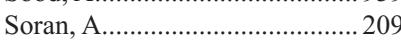

Sorensen, P....................256, 1547

Soria, JC .................................. 1612

Sorolla, A...................................... 492

Sosa, AM ……………………..... 362

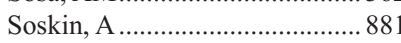

Soslow, R ……………....961, 1080

Soslow, RA.....................978, 1087

Sotgia, F ..................................... 325

Soto, C.............................. 741, 771

Soto, JL ...................................... 546

Soubrane, O............................... 1452

Soucy, G ........................................ 668,669

Soundararajan, S …..............5, 124

Soures, R ......................................... 359

Sousa-Canavez, JM …_.......... 1694

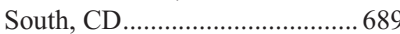

Soyupak, B ................................... 754

Spagnoli, LG ................................ 959

Spahr, L ..................................... 1460

Sparano, JA …………….... 114, 115

Spaulding, BO................. 472, 1424 ,

Spechler, SJ …………………... 559

Spector, Y ........................ 763, 1630

Spencer, HJ …………………...... 1366

Spencer, ML ................................. 670

Spyris, CT …………………..... 1739

Sreenath, T .................................... 764

Srigley, J.................................... 904

Srigley, JR .................................. 827

Srivastava, A …………..... 556, 567,

$612,639,653$

Srivastava, S............. 764, 765, 900

Srougi, M .................................... 1694

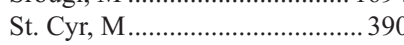

St. John, TL .................................. 366

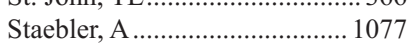

Staerkel, G.................................... 38

Staffeld-Coit, CG ……………... 1330

Staley, C ..........................672, 1399

$1443,1444,1465$

Stamatakos, M............................ 1105

Stankiewicz, E.............................. 715

Stankiewicz, P........................... 1486

Stark, D ………………………...... 394

Starostik, P ................................. 113

Stasik, CJ.................................. 1300

Stass, SA ……………………....1325

Staudt, LM .............................. 1258

Staugaitis, SM ………………….... 1487

Stearns-Kurosawa, DJ.............1331,

1339,1348

Steciuk, MR …………………....... 304

Steers, G................................... 1544

Stegall, MD …………………....1365

Stein, L .................................... 1698

Steine, E....................................1535

Steinhoff, MM................. 331, 1061

Steinkampf, JL ................................ 33

Steinmetz, J …………………....... 305

Stelow, E ……………………... 1390

Stelow, EB.................. 14, 368, 404,

$1146,1440,1461$

Stenzel, P........................................ 16

Stephan, C .......................................... 853

Stephens, AL ………………..... 1006

Stephens, JA ................................947

Stephenson, AJ .......... 879, 882, 891

Stevenson, MA.............................. 73

Stewart, D …………...... 1253, 1691

Stitzel, MR …………………...... 1462

Stockhausen, S .......................... 1329

Stoddard, GJ........................................ 1221

Stoecker, M …………………....1301

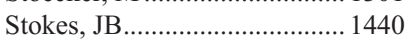

Stoler, M.................................. 987

Stoler, MH.........................362, 368

Stoll, L............................. 671, 1463

Stone, A .................................... 1153

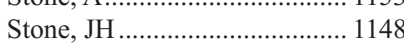

Strang, J................................... 1352

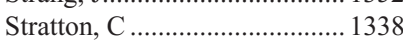

Stratton, TD ................................. 274

Streblow, R................................... 89

Strek, M................................... 1592

Streutker, CJ................................ 918

Striebel, JM ................................ 122, 259

Strosberg, J........................518, 519

Stryjewska, B ............................. 1345

Stull, WR _......................... 1248

Sturgis, CD .................................... 443

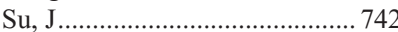

Suarez, A …………………….... 1034

Suarez, AA ……………………... 947

Suarez, Y .................................. 883

Subhawong, AP .......................... 1584

Subik, K ………………….......... 307

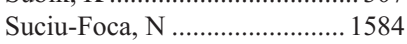

Suda, K................................... 1404

Suen, JY .............. 1125, 1136, 1534

Suescun, D ............................... 1116

Sugarbaker, D........................... 1583

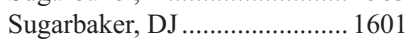

Sugita, M.................................. 1617

Sugiyama, H................................. 203

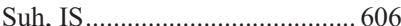

Suh, J....................................... 1596 
Sun, D …............. 1277, 1278, 1317 Sun, DI ..................................... 1133

Sun, DX..................................... 1163

Sun, L ........................... 727, 1772

Sun, M................................... 1540

Sun, P ..................................... 1046

Sun, X ……………………..... 1276

Sun, Y.................................... 1302

Sundararajan, D........................ 1114

Sung, CJ ................ 331, 392, 1048, $1054,1061,1082$

Sung, MT …………….................. 884

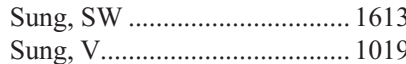

Sung, WJ ................................... 216, 794

Sung, W-J ............................... 793

Suo, ZH .................................... 238

Sur, M................................ 1093

Suriawinata, AA .... 612, 1464, 1740

Surti, U......................... 179, 1187

Susnik, B .................. 172, 213, 308

Sutherland, C............................. 1760

Sutherland, DE................... 838, 839

Suurmeijer, AJ................................ 91

Suzue, K........................ 1605, 1644

Svec, A ……............................... 840

Svensson, M......................................... 854

Svensson, MA .......... 853, 885, 1677

Swanson, PE .......... 183, 1349, 1397

Sweet, J ...................................755

Sweet, W ...........................181, 372

Swenerton, K................... 979, 1008

Swerdlow, SH …......... 1159, 1187,

1188,1199

Swindell, R............................... 1707

Swisher, SG.......................625, 626

Swisshelm, K ........................... 1166

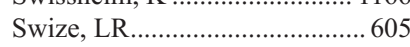

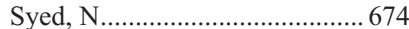

Symeonidis, A .......................... 1297

Symmans, WF......... 181, 372, 1533

Syrbu, S.................................... 1266

Syrigos, KN............................... 1717

Syrokosta, I ………………….... 1468

Szankasi, P .............................. 1159

Szpaderska, A ..................................

Szpaderska, AM ........................ 1570

Szuhai, K.................... 75, 84, 85,

$92,93,100$

\section{T}

Tabak, S...................................... 1586

Tacha, D ……………………...... 314

Tacha, DE..................................... 1758

Tafe, LJ.......................... 295, 1087

Taggart, M....................................5 575

Taghian, A ……………………..... 148

Tahan, SR .................. 444, 445, 474

Tai, MS................................... 1212

Tai, P …………………....307, 319

Tajiri, T............................ 672, 1465

Takahashi, H................................. 886

Takakuwa, Y..................................... 1149

Takase, M ......................... 1404

Takazawa, Y ............................. 1030

Takei, M .....................536, 1734

Takekoshi, S............536, 887, 1734

Takeyama, K ............................. 1280

Tallini, G ....................................2 277

Talrega, N.................................... 1249

Tam, K-F ............................... 1106

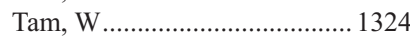

Tamagno, G............................... 1659
Tamber, N......................... 175, 240, $255,277,1506$

Tamboli, P ............................................... 862 Tamimi, R ................. 141, 142, 320 Tan, CD .................... 345, 348, 349 Tan, D ...................... 164, 576, 577, $675,676,1609,1759$

Tan, DSP ………………....254, 255

Tan, H..................................... 1329

Tan, PH ………………..... 310, 729

Tan, PL ................................... 1312

Tan, SH ……………………....... 764

Tanabe, K ……………………. 1452

Tanaka, KE............................... 1428

Tanaka, T................................. 1606, 1689,

1697, 1729

Tanas, MR

.... 94

Tang, P...................... 182, 195, 214, $307,311,319,323$

Tang, X................................... 1540 Tang, XY ................................ 887

Tanguay, S......................... 862, 876

Tanke, HJ ............................. 92, 93

Tantbirojn, P...................1574, 1575

Tao, Q................................790, 888

Tapia, O..................................... 1392

Tarco, E ……………………….... 181

Tardanico, R.............................. 722

Tasdemir, A ……………………...... 1

Tatsiou, Z ……………………........... 1011

Taube, T.................................. 1209

Tavares, R....... 687, 871, 915, 1622

Tavassoli, FA.....217, 218, 253, 285

Tavora, F …………...699, 889, 890

Tawfik, O............................265, 673

Taxy, J ........................... 805, 880

Taxy, JB........................... 24, 1110

Taylor, J ................................... 1303

Taylor, M..................................... 364

Taylor, RW ………………….......346

Tefferi, A …………………….... 1240

Teh, BT......................................... 752

Teh, YC ……………………........209

Tehsin, M .......................................... 189

Teller, L ……………………......... 866

Teman, CJ ................................ 1304

Temmins, CA ………………........384

Tench, WD .................................... 426

Teng, J ........................... 1370, 1371

Teodoro, WR …………………. 1627

Teot, LA ………………………....... 397

Teramoto, A..................................536

Terraz, S .................................... 1433

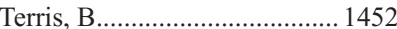

Terris, MK …………………ㄱ… 796,837

Terry, J..........................1645, 1664

Terry, PD .................................. 1290

Teruya-Feldstein, J......... 1179, 1237

Tetriakova, M............................ 1576

Tetu, B ............................................ 953

Tetzlaff, M.............................. 1147

Teverovskiy, M................................ 1682

Tewari, A _............................ 1676

Tewari, AK …………………........ 853

Thangapazham, R ...................... 764

Theis, JD ................. 350, 440, 1307 ,

1367, 1706, 1710

Theise, N .................................... 599

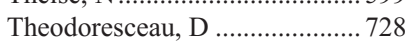

Thibodeau, SN ................483, 1070

Thiene, G..................................... 337

Thike, AA ........................................ 310

Thirabanjasak, D ........................ 1392

Thiruvaiyaru, D......................... 330,837
Thoburn, CJ................................. 500

Tholouli, E ………………….......1707

Thomas, AA …………….... 879, 891

Thomas, D ..................................222

Thomas, DG.......... 911, 1668, 1684

Thomas, P................................ 265

Thomison, JB ............................. 1665

Thomopoulos, K …………….... 1468

Thompson, PA............................. 549

Thompson, RH ............................... 696

Thomson, TM.............................. 167

Thorat, K ……………………..... 82

Thorat, MA.................................. 312

Thornburg, R............................. 913

Thung, SN ....................................... 1464

Thunnissen, FB ……………..... 1646

Thway, K........................................ 44

Tian, HS ……………………..... 745

Tian, Y............................. 313, 1519

Tibshirani, R...................1249, 1669

Tickoo, SK ...............696, 759, 792, $864,865,892,1752$

Tierno, BJ ................................ 1555

Tighiouart, M ..................................... 44

Tihan, T ……………………........ 1502

Timon, CI ................................. 1115

Tintinago, LF ……………….... 1116

Tirabosco, R ………………. 55, 95

Tiret, E ………………………..... 631

Tischler, V ................................ 1648

Tkachuk, D.................................... 904

Tobar, A.........................1375, 1701

Tochigi, N ..................................... 74

Todd, WU ...................................422

Toffolatti, L …………………........... 661

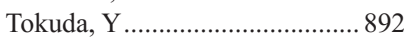

Toledo, MV ……………….......1505

Tolkoff-Rubin, N............................. 1363

Toll, AD ....................................... 1466

Tomaiuolo, G ............................ 157

Tomaszewski, J ................. 753, 767

Tomaszewski, JE.......................... 718

Tomlins, SA........................ 824, 826

Tone, A ……………………....... 1704

Toner, ME ……………………. 1115

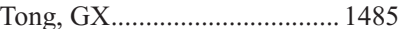

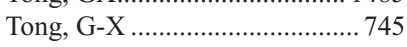

Tong, Z ....................................... 625

Toomey, C ..................................... 1234

Toor, AA....................................125

Torbenson, M ....... 1418, 1451, 1467

Torlakovic, EE ...... 674, 1225, 1664

Torne, A ....................................974

Tornos, C...................... 1050, 1087

Torres, A _................................ 1282

Torres, C.......................................................... 550

Torres, I de ................................. 893

Torres, JJ .................................... 881

Torres-Cabala, C .......................... 480

Toscano, MP.............................. 1517

Toubaji, A......................... 698, 700 ,

$813,894,895$

Tous, S..............................738, 739

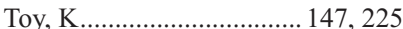

Toyokuni, S ................................1320

Tracey, D ……………………......2212

Tran, A ........................................ 952

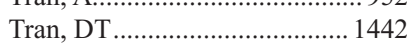

Tran-Thanh, D................. 423, 1556

Travaglini, C ………………….... 346

Travis, W....................... 1641

Travis, WD ........... 1571, 1595, 1596

Traynor, O …………………..... 1659

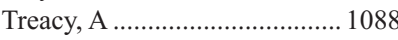

Treml, A ..................................... 1518

Tretiakova, M................... 145, 187,

$896,897,1122$

$1589,1616,1642,1644$

Trias, I …………………….......... 660

Trieu, Y ........................ 1172, 1275

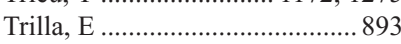

Triller, C........................................ 609

Trimarchi, F …………………..... 528

Tripathi, A …………..... 1680, 1685

Tripp, S..............................469, 1252

Tripp, SR …………………...... 122

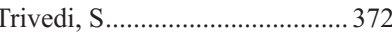

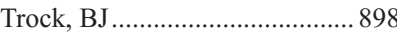

Tron, V .............................. 437, 454

Troncoso, P........ 707, 741, 771, 806

Trpkov, K .......... 877, 878, 903, 916

True, LD ................................... 899

Trunk, MJ ................................... 962

Truong, CD ....................576, 577,

$675,676,1759$

Truong, L......................... 788, 1380

Truong, LD.... 875, 919, 1690, 1771

Truskinovsky, AM..................... 1089

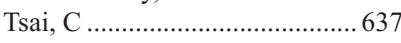

Tsamandas, A …………...691, 1412

1413,1468

Tse, GM.................................... 1503

Tseng, G .......................... 138, 1068

Tseng, LH.........................313, 1427

Tsiamalos, P .............................. 691

Tsiatis, AC ........................................ 96

Tsilidis, K .................................. 772

Tsivis, PA..................................... 314

Tsokos, M.... 809, 1568, 1569, 1779

Tsongalis, GJ ....................295, 984 ,

$1719,1737,1739$

$1740,1748,1755$

Tsota, I........................... 1412, 1413

Tsou, M-H .

1181

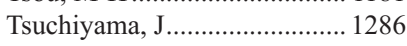

Tsuji, K....

Tsunoda, T....................................... 900

Tsuzuki, T................... 757, 901, 925

Tubbs, R ..................94, 260, 1121,

1143,1762

Tubbs, RR .............................. 1300

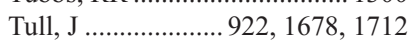

Tunnell, DL .............................. 1469

Turan, $\mathrm{O}$..................................... 537

Turbat-Herrera, EA ........ 1370, 1371

Turbiner, J …………….. 1090, 1148

Turley, H .................................. 1544

Turner, G ...................... 1305, 1760

Turner, L.................. 518, 519, 1406

Turner, LM ............................... 1708

Turner, S.................................... 1143

Tutt, A.......................................255

Tuttle, RM ………………...531, 532

Tuvin, D ………………………..... 64

Twaddell, WS............................ 1470

Twohig, M........................................... 784

Tworoger, S .....................121, 1575

Tyson, DR ………………........... 900

Tzelepi, V........................691, 1412

Tziastoudi, E ....……………….... 1011

$\mathbf{U}$

Uccella, S .................................. 1306 


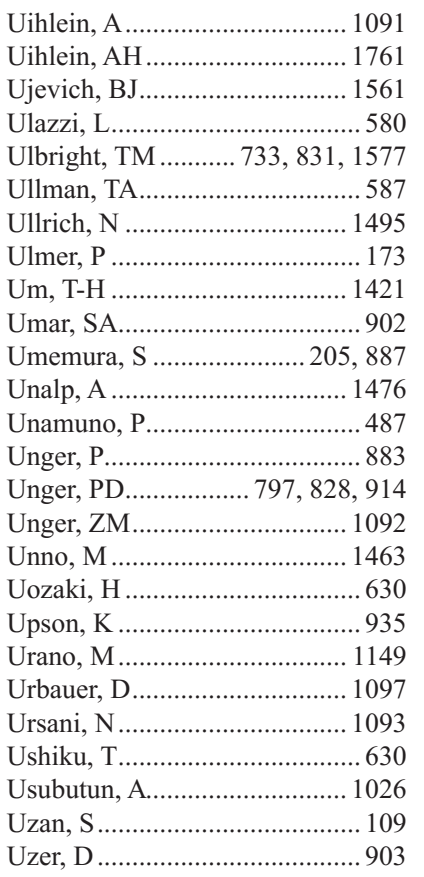

V

Vachharajani, N........................ 1422 Vadhan-Raj, S .......................... 1202 Vagenas, K ..................................... 691 Vagner, S ........................ 227, 1692 Vaickus, L.................................. 1610 Vakiani, E........ 623, 677, 678, 1391 Vakil, H ......................... 494, 1449 Val, D ……………………….... 105 Val-Bernal, JF ............................ 105 Valbuena, JR …………………….... 561 Valdez, R .................................. 1367 Valdman, A.................................... 772 Valencia-Cedillo, R …........... 107 Valentino, CM ………………....... 495 Valera, S ................................... 1160 Valera, VA …………………....... 1709 van de Rijn, M..........268, 289, 1019 Van de Rijn, M .......................... 1669 Van der Aa, M ............................. 905 Van der Kwast, TH.............904, 905 Van Eyken, P............................ 1567 van Leenders, A............................ 727 van Leeuwen, DJ........................ 1464 van Oort, IM...............................906 Van Patten, KE ............................. 679 van Pel, M ..................................... 75 Van Rhijn, BW ...........................905 Van Tuinen, P ............................ 1309 Van Vranken, JS ........................ 1749 Vance, G................................... 1676 Vang, R...........................306, 1078 , $1101,1105,1554$ Vang, RS ....................... 1037, 1060 Vanpatten, KA........................... 1762

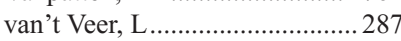
VanZee, K …ㄴ................... 326

Vargas, CM................................. 1627 Varma, M.................................... 812

Varney, RC ………………….... 1657 Varsegi, GM ................................. 424

Varsegi, MF..................................... 680

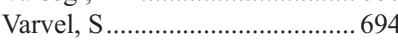
Vasco, MW …………………........703 Vasef, MA …………....... 1196, 1647 Vassallo, J..................................... 280 Vassilakopoulos, TP .................. 1186
Vatcheva, R .......................254, 255 Vauthey, J-N ………………...... 1452 Vaziri, C ..................................... 1536 Vazquez, MA....................269, 1557 Veerappan, R ........................... 1154 Vega, F ……........ 1217, 1230, 1259 Veinot, JP ....………………….... 335 Velankar, M............................... 1154 Velasco, A.................................... 492 Velasco, V ................................... 811 Velazquez, EF ..................496, 738

$739,848,881$

Velculescu, VE...…………….... 1396 Vellone, VG............................... 1102 Venick, RS................................ 1431 Venkataraman, G........................ 702 Venkataramu, C.................. 149, 250 Verardo, MR ……….............. 1763 Verbeke, SL............................97, 98 Verdu, M ……………………...... 660 Verdun Di Cartogno, L................ 240 Verhoeven, DHJ .............................. 37 Verma, SK ………………........122 Vermeer, PD ............................... 1021 Verrall, R ................................ 1338 Verstovsek, S.................1220, 1246 Viale, G ................................. 1629 Vicens, J ............ 105, 809, 866, 907 Vicini, FA ................................... 704 Victor, TA.........................443, 1760 Vidal, A ……………………….......... 660 Vieillefond, A ..................................... 881 Viera, E ....................................... 779 Vierkant, R ................................ 316 Vierkant, RA …………..... 118, 251 Vigetti, D.................................. 1417 Vigneau, C.................................... 751 Vigneswaran, W ........................ 1591 Vila, L......................................1150

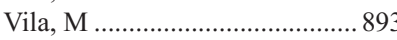

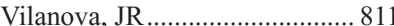
Vilarrodona, A ...............................340 Villamor, N................................ 1195 Viloria-Petit, A …………............ 117 Vincent, GA.................................... 681 Vincent-Salomon, A ..................... 185 Vinco, M ......................1668, 1684 Vinh, TN..................................... 315 Vinolas, N ................................. 1632 Virk, RK ……………………....... 497 Vishcher, D................................... 146 Visscher, D ....................................28 Visscher, DW ............ 118, 251, 316 Viswanatha, DS ......................... 1175 Viswanathan, AN ..................... 1023 Vivekanandan, P............. 1418, 1451 Vleugels, RA................................ 496 Vlotinou, H …………………......... 1297 Vogel, UF ..................................... 1764 Vogelstein, B .............................. 1396 Vogenthaler, N........................... 1335 Voight, LF …………………....... 935 Volinia, S...................................... 186 Vollmer, R _........................ 1543 Vollmer, RT …………….... 742, 743 Volynskaya, Z .............................. 317 Voralia, M.................................1225 Vortmeyer, A ................................... 941 Vose, JM ........................ 1201, 1258 Votava, HJ .................................... 498 Vrana, JA _ $\quad 60,350,440$, $1307,1367,1706,1710$ Vu, Q ……………………........ 1010 Vyas, T ……………………….... 1123 Vyas, V ...................................... 510
W

Wada, D..................................... 1308

Wada, DA ................................... 450

Wadhwani, N............................... 266

Wafai, R ............................... 433

Wagner, A...................................... 753

Wagner, D ................................... 499

Wagner, DG................... 1471, 1472

Wagner, M................................ 1765

Wagner, PL................................. 1648

Wagoner, MJ ......................... 176, 318

Waisman, J ................................. 43

Wakamatsu, A................. 695, 1552

Wakely, Jr, PE ............................ 360

Waknitz, M................... 784, 1262

Waldman, F .......................51, 128

Waldman, SA ................... 687, 943

Waldron, J ................................... 1135

Walk, E....................................... 260

Walker, JL ................................. 1108

Walker, S ................................... 12

Walter, BA................................. 1709

Walters, MP............................... 1309

Walton, D .................................. 502

Walts, A................... 395, 425, 944

Walts, AE ............... 465, 977, 1094

Wands, J ................................... 1537

Wandtke, JC .............................. 1590

Wang, BY .................................... 845

Wang, C............... 423, 1172, 1556

Wang, D ........ 36, 1538, 1556, 1772

Wang, E........................ 1177, 1301

Wang, EL........................... 538, 657

Wang, GH................................... 67

Wang, $H$.......... 552, 575, 625, 682 , $908,1198,1426,1452$

Wang, HB................................... 909

Wang, HH.................................... 669

Wang, HL ................ 472, 514, 515,

$542,564,1423$

$1424,1471,1472$

Wang, HM .......................... 575

Wang, HY.......................... 1292, 1310

Wang, J.................. 214, 263, 311,

1176,1177

Wang, JL ..................................... 319

Wang, JM ................. 195, 307, 319

Wang, JY .................................. 1700

Wang, L.................. 260, 826, 1762

Wang, LG ................................... 921

Wang, LJ ....... 871, 915, 1537, 1622

Wang, M.......................... 729, 884,

$1166,1180,1649$

Wang, MD .................................. 917

Wang, MX.................................. 1267

Wang, Q ................................... 1311

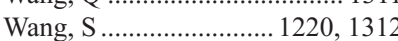

Wang, SA ...................... 1192, 1494

Wang, SAA................................ 1191

Wang, TL....................... 1015, 1016

Wang, W...... 83, 315, 700, 844, 849

Wang, W-L .................... 44, 80, 99

Wang, X ............. 69, 70, 182, 207

$311,323,370,413$

$415,1040,1649$

Wang, XM................................. 950

Wang, Y............ 320, 768, 910, 922

Wang, YE .................................. 331

Wang, YJ ..................................... 351

Wang, YZ ................................ 1645

Wang, Z .............................46, 1703

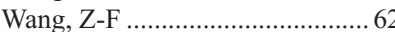

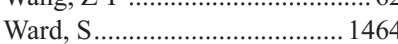

Warneke, CL
Warner, J ......................... 272, 284

Warren, K................................... 423

Warren, S........................ 441, 1270

Wartchow, E ............................. 1776

Wartenberg, M......................... 1313

Wasco, MJ....................... 911, 912

Waser, B ....................................... 610

Washington, MK ................ 692, 1437

Wasik, M .................................... 1198

Wasik, MA ................................ 1189

Wasserman, P ........................... 359

Watanabe, E ............................. 1349

Watkin, N .................................. 715

Watkin, WG.....................913, 1666

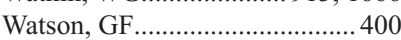

Watson, K................................. 904

Watson, L ................................... 364

Watson, MA ……......................... 304

Watson, P................................... 563

Weaver, DL .................................. 230

Weaver, K................................... 230

Webber, BA................................ 1766

Weber, A......... 590

Weber, D .................................... 1548

Weber, JA ...................................... 199

Weber, RS ................................. 1117

Webster, JA .................................. 289

Weder, W.................................... 1648

Weeden, EM.................................... 1314

Weedon, J .................................... 303

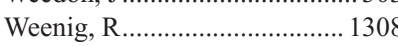

Weenig, RH............................... 440

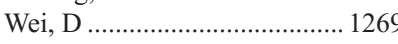

Wei, J......................................... 1031

Wei, JJ ............................ 790, 921

Wei, MQ....................................... 67

Wei, S .............................. 321, 1095

Wei, Y-C....................................... 683

Wei, JT ...................................... 873

Weidenbach, H ............................... 590

Weigelt, B............... 175, 244, 322

Weinberg, OK .............. 1315, 1316

Weinbreck, NI .......................... 1490

Weinreb, I............1127, 1151, 1152

Weinsensel, J........................... 323

Weinstein, B ............................ 1611

Weinstein, LJ................................ 662

Weinstein, RS.............................. 507

Weinstern, S ................................ 194

Weir, BA...................................... 1648

Weisenburger, D..................... 1263

Weisenburger, DD ......... 1201, 1258

Weiser, M .................................... 677

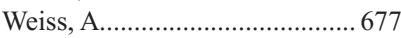

Weiss, G ................................... 727

Weiss, LM ... 263, 1176, 1177, 1215

Weiss, SW ............................. 35, 44

Welch, DR .................................... 654

Wells, JT..................................... 605

Wells, R.................................... 1434

Wells, WA...................................... 295

Welsh, CT ............................... 1523

Welton, ML .................................. 548

Wen, YH.................................... 1767

Wener, MH............................... 1750

Wenig, BM ................................ 112

West, AM …................................. 946

West, RB .................. 268, 289, 1669

Westerhoff, M ................... 684, 685

Westfall, DE .................................... 1096

Westin, S .................................. 1097

Westman, R ................................. 697

Westra, WH............................... 1145

Wexler, LH .................................. 1571

Wexner, S ................................. 552 
Weydert, JA.................... 1631, 1654 Wheeler, TM ..................... 727, 769

White, FV ...................................... 11

White, J ...................................... 149

White, S...........................202, 324

Whitelegge, JP ……………….... 291

Whiteley, L...................... 137, 1294

1513,1723

Whiteman, DC ………………...... 555

Whitman, EJ............................... 764

Whitney, KM.............................. 1428

Wick, MR ……………………... 170

Wieczorek, TJ ………………….... 363

Wiehagen, L .............................. 1358

Wierda, WG …………………... 1289

Wiernikowski, J ......................... 160

Wijers-Koster, P ............................ 85

Wilbur, DC ……...................... 426

Wilcox, R ................ 145, 684, 686, $1098,1317,1473$

Wilding, G................113, 215, 1609

Wilhorn, AJ ……………………... 30

Wilhorn, AW …………………..... 31

Wilk, M .................................... 694

Wilkinson, DS ........................... 1693

Willems, SM ………………....... 100

Williams, D .................................. 836

Williams, DL...………………. 1345

Williams, JW ........................... 1367

Williams, MD................................539

Williams, RD …………………...... 816

Williams, S................................ 1474

Williams, SR ………………...... 373

Wilson, AL ....................... 913, 1666

Wilson, AR …………………..... 1304

Wilson, L.................................... 1476

Winn, BJ........................................ 687

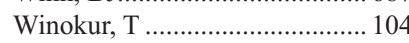

Winter, D..........................568, 581

Winter, J .................................. 1396

Wisell, J...................................... 500

Wiseman, W .............................. 1492

Wistuba, I...................... 1540, 1643

Witjes, JA ……………………........906

Witkiewicz, AK .................. 325, 1475

Witten, DM ………................ 1669

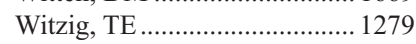

Wiweger, M.........................93, 101

Woda, B ..............................390, 466

Woda, BA............ 1243, 1494, 1542

Wohlever, K …………………. 1350

Wojcik, EM ...... 374, 379, 433, 1718

Wojweda, C............................... 1405

Wolfgang, C .............................. 1456

Wolfgang, CL ............................. 1396

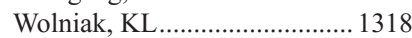

Won, KY …............................... 102

Wong, A....................................... 1249

Wong, AK …………...369, 427, 1099

Wong, C ……..............................365

Wong, G .............................................. 1503

Wong, JW ........................................ 1197

Wong, K .................................... 1256

Wong, SS.................................... 1503

Wong, V ……………………….... 423

Wong, W ............. 1363, 1364, 1369

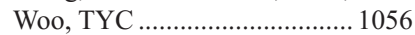

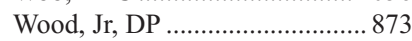

Woodard, J .......................... 89

Woods, M..................................... 770

Worman, M ............................... 207

Worsham, MJ ............................... 137

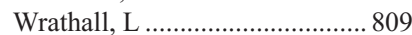

Wright, $\mathrm{H}$.................................... 136

Wright, HN..................................... 32
Wright, JL.................................... 899 Wright, JP..................................1727

Wright, TC ………………….... 1026

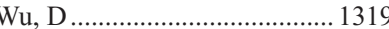

$\mathrm{Wu}, \mathrm{HY}$.....................................574

$\mathrm{Wu}, \mathrm{JM} \ldots \ldots \ldots \ldots \ldots \ldots \ldots \ldots \ldots \ldots \ldots \ldots \ldots \ldots . .500$

$\mathrm{Wu}, \mathrm{T}$........................................ 1454

Wu, TT .................... 625, 1386, 1480

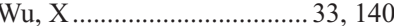

$\mathrm{Wu}, \mathrm{Y}$........................... 1492, 1533

Wurzel, J ……………………...26

Wuyts, W..................................... 93

Wynveen, C.........................326, 327

Wysocka, M .............................. 1198

\section{$\mathbf{X}$}

Xiao, G. .883

Xiao, GQ …………... 797, 828, 914

Xiao, J .............................. 571, 577

Xiao, L ............................................ 81

Xiao, LC..................................... 950

Xiao, SY …………………….....564

Xiao, W ……………………….... 674

Xiao, Y ……………….......119, 293 ,

$706,1529,1585,1639$

Xie, C ............................. 737, 872,

$1125,1153,1534$

Xie, K.............. 577, 675, 676, 1759

Xie, Q......................... 1156, 1295

Xie, QM .......................................98

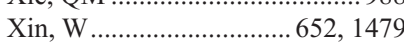

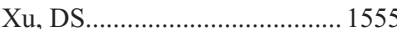

Xu, H....................... 472, 564, 688,

$1423,1424,1471$,

$1472,1590,1598$

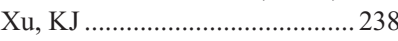

$\mathrm{Xu}, \mathrm{M}$............................. 1071, 1072

$\mathrm{Xu}, \mathrm{R} \ldots \ldots \ldots \ldots \ldots \ldots \ldots \ldots \ldots . . . . . . . . . . . . . . . . . . .1979,1407$

$\mathrm{Xu}, \mathrm{W}$............................. 1172, 1247

Xu, X................................. 199, 456

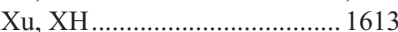

\section{Y}

Yaar, R ………………………..... 462 Yaffe, M ………………............ 1772 Yagi, Y................. 1299, 1351, 1360 Yakirevich, E..............................915 Yakoushina, T........................... 428 Yamada, S ................................... 538 Yamaguchi, H.............................. 633 Yamashita, Y …………………11320 Yan, K ……………………….... 1533

Yan, QT ……………………...... 1687 Yan, Q-T...................................... 1551 Yanagisawa, A. ..... 58

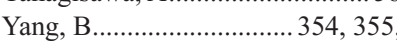
930, 931,1768

Yang, DC...................................... 351

Yang, DK ……………………..... 1636

Yang, DT ……………………..... 1769

Yang, G ................... 558, 663, 1107

Yang, GCH............................... 428

Yang, GY................................... 627, 650, 651

Yang, G-Y ................................... 637

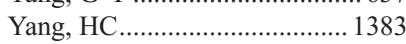

Yang, H-C ………………….... 1385

Yang, J....................................... 361

Yang, L ........................................ 131

Yang, Q .................. 499, 682, 1471, $1472,1519,1598$

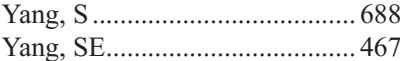

Yang, W................................... 1321 Yang, XJ.......... 752, 790, 888, 1031 Yang, XR ................................... 292 Yantiss, RK .............623, 646, 1445 Yao, H ………....... 626, 1259, 1492 Yao, J............. 675, 676, 1352, 1759 Yao, JL ……………………..... 850 Yao, T .............................................. 1404 Yao, Y …………………............ 663 Yaqo, RT …………………...... 1322 Yasin, SY ................................. 1103

Yassin, Y …………….... 1056, 1067 Yasuda, M ……………... 633, 1100 Yasuda, T............................52, 103

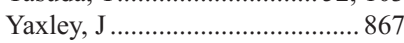
Yaziji, H ................. 182, 271, 1725 $\mathrm{Ye}, \mathrm{H}$.................................. 55, 845 Ye, Y………….... 119, 1529, 1687

Yeap, BY …………………… 1635 Yearsley, K ............... 119, 293, 1529 Yearsley, MM ....................620, 689 Yeatman, T . . . .

Yeatman, TJ.......................149, 250

Yee, H................................579, 1407

Yeh, I ............................328, 175

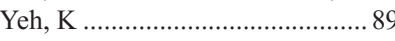

Yeh, M...................................... 1418

Yeh, MM ……............... 1397, 1414 $1462,1467,1476$

Yeldandi, AV ................... 752, 1628

Yemelyanova, A ......................... 1101

Yemelyanova, AV ......................1078

Yeo, CJ ……………………….... 1475

Yeoh, A.................................. 1312

Yeramian, A......................492, 1075

Yerian, LM …………..... 1388, 1423

Yi, ES ........................... 1634, 1640

Yildiz Aktas, IZ .......................... 1716

Yilmaz, A........... 877, 878, 903, 916

Yilmaz, F................................. 1439

Yin, CC ……………………....1276

Yin, D-T ……………………...... 1551

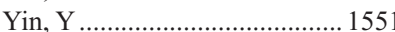

Ying, D ....................................... 1695

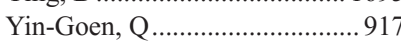

Ylip, A A................................ 676

Yoldemir, T …………………............ 966

Yonesaka, K ……………….....1585

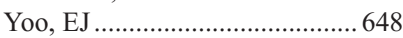

Yoo, SB …………………....... 1650

Yoon, G-S....................................595

Yoon, S-M ................................ 1421

Yoon, SO ……………………...... 690

Yopp, A....................................... 1389

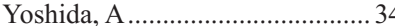

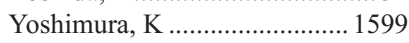

Yoshino, Y K......................... 901

Yoshizawa, A.............................. 1595

You, J...................................... 1276

Young, AN......................... 849, 917

Young, GQ …………………......... 329

Young, KH ...........1172, 1262, 1769

Young, MA................................. 448

Young, RH..........1017, 1018, 1090

Youngson, B ................................ 423

Younis, T _............................. 275

Youschak, M ....................429, 846

Yousef, GM …………………....... 918

Yousem, S .............................1358

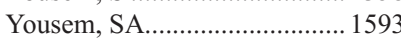

Youssef, Y .................................. 918

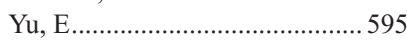

Yu, G ..............................430

Yu, GS ……………………...... 1558

$\mathrm{Yu}, \mathrm{H}$........................................... 1255

Yu, HP ........................................... 574

Yu, J.……………………..... 1651

Yu, R ……………………........ 431

Yu, SC ………………………...... 66

Yu, WM........................ 1477, 1478

Yuan, J......................................... 351

Yuldasheva, N ………………....941

Yum, SW ............................................ 332

Yun, JH................................1636

Yung, A..................................... 1232

Yung, R …………………........ 387

\section{$\mathbf{Z}$}

Zaatari, G ...

1285

Zabakis, P..................... 1412, 1413

Zabner, JL................................ 1021

Zadra, G ……………………..... 1699

Zafar, N .................................. 940

Zager, JS...................................... 471

Zagzag, D ................................... 1560

Zaidi, SY ...................................285

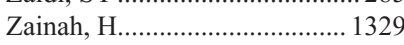

Zaino, R.......................1426

Zaki, SR .......................1333, 1347

Zakowski, M …_................1596

Zakowski, MF ..................1571, 1633

Zaloudek, CJ ..................992, 1012 ,

1029,1036

Zamo, A................................... 1711

Zanardi, C ................................. 1712

Zander, D ................................... 1426

Zander, DS ................................. 422

Zannoni, GF .............................. 1102

Zarate, R..................................... 391

Zarbo, RJ ....................... 1657, 1723

Zarineh, A................ 223, 432, 1770

Zarrin-Khameh, N......................919

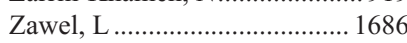

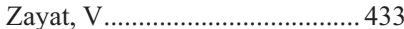

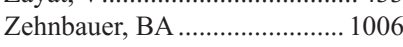

Zehnder, J.............................1315

Zehnder, JL................................ 1671

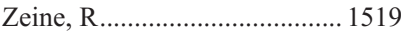

Zeldenrust, SR............................ 1307

Zellos, L ........................................ 1583

Zembowicz, A .............................. 470

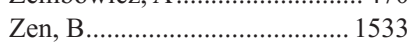

Zeng, W......................... 330, 1520

Zent, CS ………………….... 1222

Zepeda, VJ ................................. 1367

Zerbi, A ....................................... 512

Zettl, A...................................... 1205

Zeytunlu, M................................ 1439

Zhai, J.................................... 425, 434,

$435,564,576$

Zhai, Q ..................232, 1580, 1581

Zhai, QH ................................ 769

Zhai, QJ....... 788, 1065, 1450, 1771

Zhang, C........ 331, 392, 1054, 1082

Zhang, F ................................. 1547

Zhang, H .................. 307, 313, 619,

$655,737,872$

$1125,1136,1153,1534$

Zhang, HF .................................. 351

Zhang, J............................ 708, 877, $920,1132,1423$ 
416A ANNUAL MEETING ABSTRACTS

Zhang, PJ............ 40, 45, 194, 332, $456,1045,1231,1502$

Zhang, PL...................... 703, 819, $870,1025,1384$

Zhang, Q ............................... 1198

Zhang, RR ............................ 1108

Zhang, S ........................ 722, 729 $922,1323,1712$

Zhang, T ................................... 1324

Zhang, W................................ 676

Zhang, X ....................... 715, 1143

$1396,1559,1768$

Zhang, XX............................. 1248

Zhang, Y........... 8, 871, 1103, 1104

Zhang, Z ................. 137, 333, 884

Zhao, C........... 380, 436, 989, 1105

Zhao, F ................................. 1106

Zhao, J.............................. 181, 923

Zhao, JH................................... 1713

Zhao, L................................ 444

Zhao, MY ................................. 1325

Zhao, S ........................ 1249, 1257

Zhao, WQ...................................... 83

Zhao, XF ................................. 1325

Zheng, J..................................... 1107

Zheng, S .................................. 1649

Zheng, W................................. 1106

Zhong, H .................................. 1560

Zhong, M.................................. 924

Zhou, GY ….................................. 1565

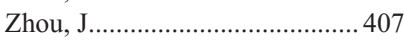

Zhou, M.................. 705, 708, 709,

$729,752,757,758$,

$773,798,818,925,926$

Zhou, Q ...................................... 574

Zhou, S..................................... 1669

Zhou, W

Zhou, X ..................................... 1753

Zhou, XK

Zhou, XM................................ 1651

Zhou, XP ...................... 1395, 1481

Zhou, Y................................... 1542

Zhou, Z ........................................ 576

Zhu, A......................................... 1452

Zhu, B ....................................... 1561

Zhu, S ............................ 1426

Zhu, X ................ 190, 1178, 1765

Zhu, Y..................................... 1387

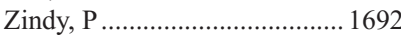

Zlotta, A....................................... 905

Zolota, V ...................................... 691

Zorludemir, S ............................. 754

Zou, X ....................................... 845

Zsikla, V ...................................... 609

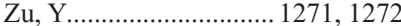

Zubovits, JT ..................... 334, 1772

Zukerberg, LR .......................... 1090

Zuluaga Toro, T......................... 692

Zuna, RE .................................. 1108

Zuo, G ............................ 741, 771

Zuo, H .......................................... 541

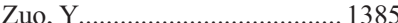

Zuo, YQ ................................... 1383

Zuo, Z ...................... 10, 446, 1109

1326,1773

Zuppan, CW ............................ 1176

Zynger, D ................................ 429

Zynger, DL.............. 746, 927, 928 